\title{
Geometric Algebra Techniques in Flux Compactifications
}

\author{
Calin Iuliu Lazaroiu, ${ }^{1}$ Elena Mirela Babalic, ${ }^{2}$ and Ioana Alexandra Coman ${ }^{3}$ \\ ${ }^{1}$ Institute for Basic Science, Center for Geometry and Physics, Pohang 790-784, Republic of Korea \\ ${ }^{2}$ Horia Hulubei National Institute for Physics and Nuclear Engineering, Department of Theoretical Physics, \\ Strada Reactorului No. 30, P.O. BOX MG-6, 077125 Magurele, Romania \\ ${ }^{3}$ DESY, Theory Group, Notkestrasse 85, Building 2a, 22607 Hamburg, Germany \\ Correspondence should be addressed to Elena Mirela Babalic; mbabalic@theory.nipne.ro
}

Received 12 May 2015; Accepted 10 September 2015

Academic Editor: Shaaban Khalil

Copyright ( 2016 Calin Iuliu Lazaroiu et al. This is an open access article distributed under the Creative Commons Attribution License, which permits unrestricted use, distribution, and reproduction in any medium, provided the original work is properly cited. The publication of this article was funded by SCOAP . $^{3}$

\begin{abstract}
We study "constrained generalized Killing (s)pinors," which characterize supersymmetric flux compactifications of supergravity theories. Using geometric algebra techniques, we give conceptually clear and computationally effective methods for translating supersymmetry conditions into differential and algebraic constraints on collections of differential forms. In particular, we give a synthetic description of Fierz identities, which are an important ingredient of such problems. As an application, we show how our approach can be used to efficiently treat $\mathcal{N}=1$ compactification of $M$-theory on eight manifolds and prove that we recover results previously obtained in the literature.
\end{abstract}

\section{Introduction}

A fundamental problem in the study of flux compactifications of $M$-theory and string theory is to give efficient geometric descriptions of supersymmetric backgrounds in the presence of fluxes. This leads, in particular cases, to beautiful connections $[1,2]$ with the theory of $G$-structures, while in more general situations it translates to difficult mathematical problems involving novel geometric realizations of supersymmetry algebras (see [3-6] for some examples).

When approaching this subject, one may be struck by the somewhat ad hoc nature of the methods usually employed, which signals a lack of unity in the current understanding of the subject. This is largely due to the intrinsic difficulty in finding unifying principles while keeping computational complexity under control. In particular, one confronts the lack of general and structurally clear descriptions of Fierz identities, the fact that phenomena and methods which are sometimes assumed to be "generic" turn out, upon closer inspection, to be relevant only under simplifying assumptions, and the insufficient mathematical development of the subject of "spin geometry [7] in the presence of fluxes."
The purpose of this paper is to draw attention to the fact that many of the issues mentioned above can be resolved using ideas inspired by a certain incarnation of the theory of Clifford bundles known as "geometric algebra," which goes back to $[8,9]$ (see also [10-14] for an introduction)-an approach which provides a powerful language and efficient techniques, thus affording a more unified and systematic description of flux compactifications and of supergravity and string compactifications in general. In particular, we show that the geometric analysis of supersymmetry conditions for flux backgrounds (including the algebra of those Fierz identities relevant for the analysis) can be formulated efficiently in this language, thereby uncovering structure whose implications have remained largely unexplored. We mention here that our methods have a (nontrivial) connection with the Gstructure and exceptional generalized geometry approaches, which were previously shown to be useful when studying flux compactifications. This connection will be discussed at length in a different publication.

Though the scope and applications of our approach are much wider, we will focus here on the study of what we call "constrained generalized Killing (CGK) (s)pinor equations," 
which distill the mathematical description of supersymmetry conditions for flux backgrounds. A constrained generalized Killing (s)pinor is simply a (s)pinor satisfying conditions of the type $D_{m} \xi=Q_{1} \xi=\cdots=Q_{\chi} \xi=0$, where $D_{m}=\nabla_{m}^{S}+A_{m}$ is some connection on a bundle $S$ of (s)pinors (which generally differs from the connection $\nabla_{m}^{S}$ induced on $S$ by the Levi-Civita connection $\nabla_{m}$ of the underlying pseudo-Riemannian manifold) while $Q_{j}$ are some globally defined endomorphisms of $S$. Such equations are abundant in flux compactifications of supergravity (see, e.g., $[15,16]$ ), where $\xi$ is the internal part of a supersymmetry generator while the equations themselves are the conditions that the compactification preserves the supersymmetry generated by $\xi$. The quantities $A_{m}$ and $Q_{j}$ are then certain algebraic combinations of gamma matrices with coefficients dependent on the metric and fluxes. An example with a single algebraic constraint $Q \xi=0$ (arising in a compactification of elevendimensional supergravity) is discussed in Section 6, which the reader can consult first as an illustration motivating the formal developments taken up in the rest of the paper.

Using geometric algebra techniques, we show how such supersymmetry conditions can be translated efficiently and briefly into a system of differential and algebraic constraints for a collection of inhomogeneous differential forms expressed as (s)pinor bilinears, thus displaying the underlying structure in a form which is conceptually clear as well as highly amenable to computation. The conditions which we obtain on differential forms provide a generalization of the well-known theory of Killing forms, which could be studied in more depth through methods of Kähler-Cartan theory [17]-even though we will not pursue that avenue in the present work. We also touch on our implementation of this approach using various symbolic computation systems.

As an example, Section 6 applies such techniques to the study of flux compactifications of $M$-theory on eight manifolds preserving $\mathcal{N}=1$ supersymmetry in 3 dimensionsa class of solutions which was analyzed through direct methods in $[3,4]$. In that setting, we have a single algebraic condition $Q \xi=0$, with $Q=(1 / 2) \gamma^{m} \partial_{m} \Delta-$ $(1 / 288) F_{m p q r} \gamma^{m p q r}-(1 / 6) f_{p} \gamma^{p} \gamma^{(9)}-\kappa \gamma^{(9)}$ and $A_{m}=$ $(1 / 4) f_{p} \gamma_{m}{ }^{p} \gamma^{(9)}+(1 / 24) F_{m p q r} \gamma^{p q r}+\kappa \gamma_{m} \gamma^{(9)}$. We show how our methods can be used to recover the results of [3] in a synthetic and computationally efficient manner, while giving a more complete and general analysis. We express all equations in terms of certain combinations of iterated contractions and wedge products which are known as "generalized products" and whose conceptual role and origin is explained in Section 3. The reader can, at this point, pause to take a look at Section 6.2, which should provide an illustration of the techniques developed in this paper.

The paper is organized as follows. In Section 2, we define and discuss constrained generalized Killing (s)pinors. In Section 3, we recall the geometric algebra description of Clifford bundles as Kähler-Atiyah bundles while in Section 4 we explain how pinor bundles are described in this approach. Using our realization of spin geometry, Section 5 presents a synthetic formulation of Fierz rearrangement identities for pinor bilinears, which encodes identities involving four pinors through certain quadratic relations holding in (a certain subalgebra of) the Kähler-Atiyah algebra of the underlying manifold. We also reformulate the constrained generalized Killing pinor equations in this language and discuss some aspects of the differential and algebraic structure resulted from this analysis, thereby extending the well-known theory of Killing forms. In Section 6, we apply this formalism to the study of $\mathcal{N}=1$ compactification of $M$-theory on eight manifolds. We conclude in Section 7 with a few remarks on further directions. Appendix summarize various technical details and make contact with previous work. The physicsoriented reader can start with Section 6, before delving into the technical and theoretical details of the other sections.

Notations. We let $\mathbb{K}$ denote one of the fields $\mathbb{R}$ or $\mathbb{C}$ of real or complex numbers. We work in the smooth differential category, so all manifolds, vector bundles, maps, morphisms of bundles, differential forms, and so forth are taken to be smooth. We further assume that our connected and smooth manifolds $M$ are paracompact and of finite Lebesgue dimension, so that we have partitions of unity of finite covering dimension subordinate to any open cover. If $V$ is a $\mathbb{K}$-vector bundle over $M$, we let $\Gamma(M, V)$ denote the space of smooth $\left(\mathscr{C}^{\infty}\right)$ sections of $V$. We also let $\operatorname{End}(V)=\operatorname{Hom}(V, V)=$ $V \otimes V^{*}$ denote the $\mathbb{K}$-vector bundle of endomorphisms of $V$, where $V^{*}=\operatorname{Hom}\left(V, \mathcal{O}_{\mathbb{K}}\right)$ is the dual vector bundle to $V$ while $\mathcal{O}_{\mathbb{K}}$ denotes the trivial $\mathbb{K}$-line bundle on $M$. The unital ring of smooth $\mathbb{K}$-valued functions defined on $M$ is denoted by $\mathscr{C}^{\infty}(M, \mathbb{R})=\Gamma\left(M, \mathscr{O}_{\mathbb{K}}\right)$. The tensor product of $\mathbb{K}$-vector spaces and $\mathbb{K}$-vector bundles is denoted by $\otimes$, while the tensor product of modules over $\mathscr{C}^{\infty}(M, \mathbb{K})$ is denoted by $\otimes_{\mathscr{C}^{\infty}(M, \mathbb{R})}$; hence $\Gamma\left(M, V_{1} \otimes V_{2}\right)=\Gamma\left(M, V_{1}\right) \otimes_{\mathscr{C}^{\infty}(M, \mathbb{R})} \Gamma\left(M, V_{2}\right)$. Setting $T_{\mathbb{K}} M \stackrel{\text { def }}{=} T M \otimes \mathcal{O}_{\mathbb{K}}$ and $T_{\mathbb{K}}^{*} M \stackrel{\text { def }}{=} T^{*} M \otimes \mathcal{O}_{\mathbb{K}}$, the space of $\mathbb{K}$ valued smooth inhomogeneous globally defined differential forms on $M$ is denoted by $\Omega_{\mathbb{K}}(M) \stackrel{\text { def }}{=} \Gamma\left(M, \wedge T_{\mathbb{K}}^{*} M\right)$ and is a $\mathbb{Z}$-graded module over the commutative ring $\mathscr{C}^{\infty}(M, \mathbb{R})$. The fixed rank components of this graded module are denoted by $\Omega_{\mathbb{K}}^{k}(M)=\Gamma\left(M, \wedge^{k} T_{\mathbb{K}}^{*} M\right)(k=0 \cdots d$, where $d$ is the dimension of $M)$.

The kernel and image of any $\mathbb{K}$-linear map $T: \Gamma(M$, $\left.V_{1}\right) \rightarrow \Gamma\left(M, V_{2}\right)$ will be denoted by $\mathscr{K}(T)$ and $\mathscr{I}(T)$; these are $\mathbb{K}$-linear subspaces of $\Gamma\left(M, V_{1}\right)$ and $\Gamma\left(M, V_{2}\right)$, respectively. In the particular case when $T$ is a $\mathscr{C}^{\infty}(M, \mathbb{R})$-linear map (i.e., when it is a morphism of $\mathscr{C}^{\infty}(M, \mathbb{R})$-modules), the subspaces $\mathscr{K}(T)$ and $\mathscr{I}(T)$ are $\mathscr{C}^{\infty}(M, \mathbb{R})$-submodules of $\Gamma\left(M, V_{1}\right)$ and $\Gamma\left(M, V_{2}\right)$, respectively-even in those cases when $T$ is not induced by any bundle morphism from $V_{1}$ to $V_{2}$. We always denote a morphism $f: V_{1} \rightarrow V_{2}$ of $\mathbb{K}$-vector bundles and the $\mathscr{C}^{\infty}(M, \mathbb{R})$-linear map $\Gamma\left(M, V_{1}\right) \rightarrow \Gamma\left(M, V_{2}\right)$ induced by it between the modules of sections by the same symbol. Because of this convention, we clarify that the notations $\mathscr{K}(f) \subset$ $\Gamma\left(M, V_{1}\right)$ and $\mathscr{I}(f) \subset \Gamma\left(M, V_{2}\right)$ denote the kernel and the image of the corresponding map on sections $\Gamma\left(M, V_{1}\right) \stackrel{f}{\rightarrow}$ $\Gamma\left(M, V_{2}\right)$, which in this case are $\mathscr{C}^{\infty}(M, \mathbb{R})$-submodules of $\Gamma\left(M, V_{1}\right)$ and $\Gamma\left(M, V_{2}\right)$, respectively. In general, there does not exist either any subbundle $\operatorname{ker} f$ of $V_{1}$ such that $\mathscr{K}(f)=\Gamma(M, \operatorname{ker} f)$ or any subbundle $\operatorname{im} f$ of $V_{2}$ such that 
$\mathscr{I}(f)=\Gamma(M, \operatorname{im} f)$-though there exist sheaves $\operatorname{ker} f$ and $\operatorname{im} f$ with the corresponding properties.

Given a pseudo-Riemannian metric $g$ on $M$ of signature $(p, q)$, we let $\left(e_{a}\right)_{a=1 \ldots d}$ (where $\left.d=\operatorname{dim} M\right)$ denote a local frame of $T M$, defined on some open subset $U$ of $M$. We let $e^{a}$ be the dual local coframe (= local frame of $T^{*} M$ ), which satisfies $e^{a}\left(e_{b}\right)=\delta_{b}^{a}$ and $\hat{g}\left(e^{a}, e^{b}\right)=g^{a b}$, where $\left(g^{a b}\right)$ is the inverse of the matrix $\left(g_{a b}\right)$. The contragradient frame $\left(e^{a}\right)^{\sharp}$ and contragradient coframe $\left(e_{a}\right)_{\sharp}$ are given by

$$
\begin{aligned}
& \left(e^{a}\right)^{\sharp}=g^{a b} e_{b}, \\
& \left(e_{a}\right)_{\sharp}=g_{a b} e^{b},
\end{aligned}
$$

where the $\sharp$ subscript and superscript denote the (mutually inverse) musical isomorphisms between $T_{\mathbb{K}} M$ and $T_{\mathbb{K}}^{*} M$ given, respectively, by lowering and raising indices with the metric $g$. We set $e^{a_{1} \cdots a_{k}} \stackrel{\text { def }}{=} e^{a_{1}} \wedge \cdots \wedge e^{a_{k}}$ and $e_{a_{1} \cdots a_{k}} \stackrel{\text { def }}{=} e_{a_{1}} \wedge \cdots \wedge$ $e_{a_{k}}$ for any $k=0 \cdots d$. A general $\mathbb{K}$-valued inhomogeneous form $\omega \in \Omega_{\mathbb{}}(M)$ expands as follows:

$$
\omega=\sum_{k=0}^{d} \omega^{(k)}=_{U} \sum_{k=0}^{d} \frac{1}{k !} \omega_{a_{1} \cdots a_{k}}^{(k)} e^{a_{1} \cdots a_{k}},
$$

where the symbol $=_{U}$ means that the equality holds only after restriction of $\omega$ to $U$ and where we used the expansion:

$$
\omega^{(k)}=_{U} \frac{1}{k !} \omega_{a_{1} \cdots a_{k}}^{(k)} e^{a_{1} \cdots a_{k}}
$$

The locally defined smooth functions $\omega_{a_{1} \cdots a_{k}}^{(k)} \in \mathscr{C}^{\infty}(U, \mathbb{K})$ (the "strict coefficient functions" of $\omega$ ) are completely antisymmetric in $a_{1} \cdots a_{k}$. Given a pinor bundle on $M$ with underlying fiberwise representation $\gamma$ of the Clifford bundle of $T_{\mathbb{K}}^{*} M$, the corresponding gamma "matrices" in the coframe $e^{a}$ are denoted by $\gamma^{a} \stackrel{\text { def }}{=} \gamma\left(e^{a}\right)$, while the gamma matrices in the contragradient coframe $\left(e_{a}\right)_{\sharp}$ are denoted by $\gamma_{a} \stackrel{\text { def }}{=}$ $\gamma\left(\left(e_{a}\right)_{\sharp}\right)=g_{a b} \gamma^{b}$. We will occasionally assume that the frame $\left(e_{a}\right)$ is pseudo-orthonormal in the sense that $e_{a}$ satisfy

$$
g\left(e_{a}, e_{b}\right)\left(=g_{a b}\right)=\eta_{a b},
$$

where $\left(\eta_{a b}\right)$ is a diagonal matrix with $p$ diagonal entries equal to +1 and $q$ diagonal entries equal to -1 .

\section{Constrained Generalized Killing (S)Pinors}

The Basic Setup. Let $(M, g)$ be a connected pseudoRiemannian manifold (assumed to be smooth and paracompact) of dimension $d=p+q$, where $p$ and $q$ are, respectively, the numbers of positive and negative eigenvalues of $g$. We endow the cotangent bundle $T^{*} M$ with the metric $\hat{g}$ induced by $g$. Setting $\mathbb{K}=\mathbb{R}$ or $\mathbb{C}$, we similarly endow the bundle $T_{\mathbb{K}}^{*} M \stackrel{\text { def }}{=} T^{*} M \otimes \mathcal{O}_{\mathbb{K}}$ with the metric $\widehat{g}_{\mathbb{K}}$ induced by extension of scalars. Of course, we have $T_{\mathbb{R}}^{*} M=T^{*} M$ and $\widehat{g}_{\mathbb{R}}=$ $\hat{g}$. Let $\mathrm{Cl}\left(T_{\mathbb{K}}^{*} M\right)=\mathrm{Cl}\left(T^{*} M\right) \otimes \mathcal{O}_{\mathbb{K}}$ be the Clifford bundle defined by $T_{\mathbb{K}}^{*} M$-when the latter is endowed with the metric given above. The fiber of $\mathrm{Cl}\left(T_{\mathbb{K}}^{*} M\right)$ at a point $x \in M$ is the Clifford algebra $\mathrm{Cl}\left(T_{\mathbb{K}, x}^{*} M\right)=\mathrm{Cl}\left(T_{x}^{*} M\right) \otimes_{\mathbb{R}} \mathbb{K}$ of the quadratic vector space $\left(T_{\mathbb{K}, x}^{*}, \widehat{g}_{\mathbb{K}, x}\right)$, where $T_{\mathbb{K}, x}^{*} \stackrel{\text { def }}{=} T_{x}^{*} M \otimes_{\mathbb{R}} \mathbb{K}$ and $\widehat{g}_{\mathbb{K}, x}$ denotes the $\mathbb{K}$-valued bilinear pairing induced by $\hat{g}_{x}$. The even Clifford bundle $\mathrm{Cl}^{\mathrm{ev}}\left(T_{\mathbb{K}}^{*} M\right)$ over $\mathbb{K}$ is the subbundle of algebras of $\mathrm{Cl}\left(T_{\mathbb{K}}^{*} M\right)$ whose fibers are the even subalgebras $\mathrm{Cl}^{\mathrm{ev}}\left(T_{\mathbb{K}, x}^{*} M\right) \subset \mathrm{Cl}\left(T_{\mathbb{K}, x}^{*} M\right)$. Our point of view on (s)pinor bundles is that taken in [18]. Namely, we define a bundle of $\mathbb{K}$-pinors over $M$ to be a $\mathbb{K}$-vector bundle $S$ over $M$ which is a bundle of modules over the Clifford bundle $\mathrm{Cl}\left(T_{\mathbb{K}}^{*} M\right)$. Similarly, a bundle of $\mathbb{K}$-spinors is a bundle of modules over the even Clifford bundle $\mathrm{Cl}^{\mathrm{ev}}\left(T_{\mathbb{K}}^{*} M\right)$. Of course, a bundle of $\mathbb{K}$-pinors is automatically a bundle of $\mathbb{K}$-spinors. Hence any pinor is naturally a spinor but the converse need not hold. In this paper, we focus on the case of pinors. A pinor bundle $S$ will be called a pin bundle if the underlying fiberwise representation of $\mathrm{Cl}\left(T_{\mathbb{K}}^{*} M\right)$ is irreducible, that is, if each of the fibers of $S$ is a simple module over the corresponding fiber of the Clifford bundle. Similarly, a spin bundle is a spinor bundle for which the underlying fiberwise representation of $\mathrm{Cl}^{\mathrm{ev}}\left(T_{\mathbb{K}}^{*} M\right)$ is irreducible. Later on, we will sometimes denote $g_{\mathbb{K}}$ by $g$, and so forth, in order to simplify notation.

Remark 1. Physics terminology is often imprecise with the distinction between spinors and pinors which we are making here and throughout this paper. Physically, one typically assumes that $(M, g)$ is both oriented and time-oriented and one is concerned with objects transforming in representations of the orthochronous part $\operatorname{Spin}^{\uparrow}(p, q)$ of the spin group $\operatorname{Spin}(p, q)$ and thus in vector bundles associated with a principal bundle with fiber $\operatorname{Spin}^{\uparrow}(p, q)$ which is a double cover of the principal $\mathrm{SO}^{\uparrow}(p, q)$-bundle consisting of those pseudo-orthonormal frames of $(M, g)$ which are both oriented and time-oriented. Due to the issue of timeorientability, what matters in many physics applications is not a spin structure in the standard mathematical sense (see [19] for a recent discussion with applications to string theory) but rather a "time-oriented" spin structure.

Constrained Generalized Killing (S)Pinors. Let us fix a $\mathbb{K}$-pinor or $\mathbb{K}$-spinor bundle $S$ over $M$, a linear connection $D$ on $S$, and a finite collection of bundle endomorphisms $Q_{1}, \ldots, Q_{\chi} \in$ $\Gamma(M, \operatorname{End}(S))$.

Definition 2. A constrained generalized Killing (CGK) (s)pinor over $M$ is a section $\xi \in \Gamma(M, S)$ which satisfies the constrained generalized Killing (s)pinor equations $D \xi=Q_{1} \xi=\cdots=$ $Q_{\chi} \xi=0$. We say that $D \xi=0$ is the $D$-flatness or generalized Killing (GK) (s)pinor equation satisfied by $\xi$ while $Q_{1} \xi=$ $\cdots=Q_{\chi} \xi=0$ are the algebraic constraints (or Q-constraints) satisfied by $\xi$.

When the algebraic constraints are trivial $(\chi=0$ or, equivalently, when all $Q_{j}$ vanish), one deals with the generalized Killing (GK) spinor equation $D \xi=0$. Since $D$ can be written as the sum $\nabla^{S}+A$ of the spinorial connection $\nabla^{S}$ induced on $S$ by the Levi-Civita connection of $(M, g)$ and an End $(S)$-valued one-form $A$ on $M$, the GK (s)pinor equations 
can be viewed as a deformation of the parallel (s)pinor equation $\nabla^{S} \xi=0$, the deformation being parameterized by $A$. Our terminology is inspired by the fact that the choice $A_{m}=-\lambda \gamma_{m}$ (with $\lambda$ a real parameter and $\gamma^{m} \in \Gamma(M, \operatorname{End}(S)$ ) the gamma "matrices" in some local coframe of $(M, g)$ ) leads to the ordinary Killing (s)pinor equations $\nabla_{m} \xi=\lambda \gamma_{m} \xi$.

Remark 3. In flux compactifications of supergravity, backgrounds admitting constrained generalized Killing spinors can be used to construct supersymmetric compactifications, provided that the equations of motion for all fields present in the background are also satisfied.

Connection to Supergravity and String Theories. Constrained generalized Killing (s)pinors arise naturally in supergravity and string theory. In particular, they arise in supersymmetric flux compactifications of string theory, $M$-theory, and various supergravity theories. In such setups, $\xi$ is a (s)pinor of spin $1 / 2$ defined on the background pseudo-Riemannian manifold and corresponds to the generator of supersymmetry transformations of the underlying supergravity action (or string theory effective action) while the constrained generalized Killing (s)pinor equations are the conditions that the supersymmetry generated by $\xi$ is preserved by the background. The connection $D$ on $S$ and the endomorphisms $Q_{j}$ are fixed by the precise data of the background, that is, by the metric and fluxes defining that background. For example, the supersymmetry equations of eleven-dimensional supergravity involve the supercovariant connection $D$, which acts on sections of the bundle $S$ of Majorana spinors (a.k.a. real pinors) defined in eleven dimensions; this corresponds to the differential constraint $D \xi=0$, without any algebraic constraint. When considering a compactification of elevendimensional supergravity down to a lower-dimensional space admitting Killing (s)pinors, the internal part (which now plays the role of $\xi$ ) of the generator of the supersymmetry variation is a section of some bundle of (s)pinors (which now plays the role of $S$ ) defined over the internal space, while the condition of preserving the supersymmetry generated by the tensor product of this internal generator and some Killing (s)pinor of the noncompact part of the background induces a differential (generalized Killing) constraint as well as an algebraic constraint for the internal part of the supersymmetry generator. A specific example arising from elevendimensional supergravity is discussed in Section 6 below. Similarly, the supersymmetry equations for IIA supergravity in ten dimensions (with Minkowski signature) can be written in terms of a supercovariant connection $D$ defined on the real vector bundle $S$ of Majorana spinors (a.k.a. real pinors) in ten dimensions and an endomorphism $Q$ of $S$; we have $S=$ $S^{+} \oplus S^{-}$where $S^{ \pm}$are the bundles of Majorana-Weyl spinors of positive and negative chirality. The condition $D \xi=0$ for the supersymmetry generator (a section $\xi$ of $S$, with positive and negative chirality components $\xi_{ \pm}$-which are sections of $S^{ \pm}$-such that $\xi=\xi_{+}+\xi_{-}$) is the requirement that the supersymmetry variation of the gravitino vanishes, while the condition $Q \xi=0$ encodes vanishing of the supersymmetry variation of the dilatino. When considering compactifications on some internal space down to some space admitting Killing (s)pinors, $\xi$ is replaced by its internal part (a section of some (s)pinor bundle-which now plays the role of $S$ defined on the compactification space) while $D$ induces a connection defined on this internal (s)pinor bundle as well as a further algebraic constraint-thereby leading once again to a system of equations of constrained generalized Killing type, which is now defined on the internal space. Finally, the supersymmetry equations for type IIB supergravity in ten dimensions (with Minkowski signature) can be formulated ${ }^{1}$ (see, e.g., [16]) in terms of sections of the real vector bundle $S=S^{+} \oplus S^{+}$of Majorana-Weyl spinor doublets, with a supercovariant connection $D$ defined on this bundle as well as two endomorphisms $Q_{1}, Q_{2}$ of $S$. The condition $D \xi=0$ for sections $\xi$ of $S$ is the requirement that the supersymmetry variation of the gravitino vanishes in the background, while the conditions $Q_{1} \xi=Q_{2} \xi=0$ are, respectively, the requirements that the supersymmetry variations of the axionino and dilatino vanish. When considering a compactification down from ten dimensions, the constraints $Q_{1} \xi=Q_{2} \xi=0$ descend to similar constraints for the internal part of $\xi$, while the constraint $D \xi=0$ induces both a differential and an algebraic constraint for the internal part; hence the compactification procedure produces a differential constraint while increasing the number of algebraic constraints, the resulting equations being again of constrained generalized Killing type, but formulated for sections of some bundle of (s)pinors defined over the internal space of the compactification.

Some Mathematical Observations. Let us for simplicity consider the case of a single algebraic constraint $(Q \xi=0)$. Let $\mathscr{K}(Q)$ denote the $\mathscr{C}^{\infty}(M, \mathbb{R})$-submodule of smooth solutions to the equation $Q \xi=0$ and let $\mathscr{K}(D)$ denote the $\mathbb{K}$-vector subspace of smooth solutions to the equation $D \xi=0$. Then the $\mathbb{K}$-vector subspace $\mathscr{K}(D, Q)$ of smooth solutions to the CGK spinor equations equals the intersection $\mathscr{K}(D) \cap \mathscr{K}(Q)$. In general, the dimension of the subspace $\operatorname{ker}\left(Q_{x}\right) \subset S_{x}$ of the fiber of $S$ at a point $x$ may jump as $x$ varies inside $M$, so $Q$ does not admit a subbundle of $S$ as its kernel (in fact, this is one reason why smooth vector bundles do not form an Abelian category) - even though it does admit a kernel in the category of sheaves over the ringed space associated with $M$. On the other hand, a simple argument ${ }^{2}$ using parallel transport shows that any linearly independent (over $\mathbb{K}$ ) collection of smooth solutions $\xi_{1}, \ldots, \xi_{s}$ of the generalized Killing (s)pinor equation must be linearly independent everywhere; that is, the vectors $\xi_{1}(x), \ldots, \xi_{s}(x)$ must be linearly independent in the fiber $S_{x}$ for any point $x$ of $M$. In particular, there exists a $\mathbb{K}$-vector subbundle $S_{D}$ of $S$ such that $\operatorname{rk}_{\mathbb{K}} S_{D}=\operatorname{dim}_{\mathbb{K}} \mathscr{K}(D)$ and such that $\Gamma\left(M, S_{D}\right)=\mathscr{K}(D) \otimes_{\mathbb{K}} \mathscr{C}^{\infty}(M, \mathbb{R})$; in fact, any basis of the space of solutions $\mathscr{K}(D)$ of the generalized Killing (s)pinor equations provides a global frame for $S_{D}$ (which, therefore, must be a trivial vector bundle). Since the restriction of $D$ to $S_{D}$ is flat, the bundle $S_{D}$ is sometimes referred to as "the $D$-flat vector subbundle of $S$." The condition that the generalized Killing (s)pinor equations admit exactly $s$ linearly independent solutions over $\mathbb{K}$ (i.e., the condition $\operatorname{dim}_{\mathbb{K}} \mathscr{K}(D)=s$ ) amounts to the requirement that $S_{D}$ has rank $s$; in particular, this imposes well-known topological constraints on $S$. A similar argument shows 
that there exists a (topologically trivial) $\mathbb{K}$-vector subbundle $S_{D, \mathrm{Q}} \subset S_{D} \subset S$ such that $\mathrm{rk}_{\mathbb{K}} S_{D, \mathrm{Q}}=\operatorname{dim}_{\mathbb{K}} \mathscr{K}(D, Q)$ and such that $\Gamma\left(M, S_{D, Q}\right)=\mathscr{K}(D, Q) \otimes_{\mathbb{K}} \mathscr{C}^{\infty}(M, \mathbb{R})$.

As mentioned in the introduction, a basic problem in the analysis of flux compactifications (which is also of mathematical interest in its own right) is to find efficient methods for translating constrained generalized Killing (s)pinor equations for some collection $\xi_{1}, \ldots, \xi_{s}$ of sections of $S$ into a system of algebraic and differential conditions for differential forms which are constructed as bilinears in $\xi_{1}, \ldots, \xi_{s}$. In Section 5, we show how the geometric algebra formalism can be used to provide an efficient and conceptually clear solution to this problem. Before doing so, however, we have to recall the basics of the geometric algebra approach to spin geometry, which we proceed to do next.

\section{The Kähler-Atiyah Bundle of a Pseudo- Riemannian Manifold}

This section lays out the basics of the geometric algebra formalism and develops some specialized aspects which will be needed later on. In Sections 3.1 and 3.2, we start with the Clifford bundle of the cotangent bundle of a pseudoRiemannian manifold $(M, g)$, viewed as a bundle of unital and associative-but noncommutative-algebras which is naturally associated with $(M, g)$. The basic idea of "geometric algebra" is to use a certain isomorphic realization of the Clifford bundle in which the underlying vector bundle is identified with the exterior bundle of $M$. In this realization, the multiplication of the Clifford bundle transports to a fiberwise multiplication of the exterior bundle; when endowed with this associative but noncommutative multiplication, the exterior bundle becomes a bundle of associative algebras known as the Kähler-Atiyah bundle. In turn, the noncommutative multiplication of the Kähler-Atiyah bundle induces an associative but noncommutative multiplication (which we denote by $\diamond$ and call the geometric product) on inhomogeneous differential forms. The resulting associative algebra is known as the Kähler-Atiyah algebra of $(M, g)$ and can be viewed as a certain deformation of the exterior algebra which is parameterized by the metric $g$ of $M$. The Kähler-Atiyah algebra is an associative and unital algebra over the commutative and unital ring $\mathscr{C}^{\infty}(M, \mathbb{K})$ of smooth $\mathbb{K}$ valued functions defined on $M$-so in particular it is a $\mathbb{K}$ algebra upon considering the embedding $\mathbb{K} \subset \mathscr{C}^{\infty}(M, \mathbb{K})$ which is defined by associating with each element of $\mathbb{K}$ the corresponding constant function. The geometric product has an expansion in terms of so-called "generalized products," which form a collection of binary operations acting on inhomogeneous forms. In turn, the generalized products can be described as certain combinations of contractions and wedge products. The expansion of the geometric product into generalized products can be interpreted (under certain global conditions on $(M, g))$ as a form of "partial quantization" of a spin system-the role of the Planck constant being played by the inverse of the overall scale of the metric. In this interpretation, the Kähler-Atiyah algebra is the quantum algebra of observables while the geometric product is the noncommutative composition of quantum observables; the classical limit corresponds to taking the scale of the metric to infinity while the expansion of the geometric product into generalized products can be viewed as a semiclassical expansion. In the classical limit, the geometric product reduces to the wedge product and the Kähler-Atiyah algebra reduces to the exterior algebra of $M$, which plays the role of the classical algebra of observables. Section 3.3 discusses certain (anti-)automorphisms of the Kähler-Atiyah algebra which will be used intensively later on while Section 3.4 gives some properties of the left and right multiplication operators in this algebra. In Section 3.5, we give a brief discussion of the decomposition of an inhomogeneous form into parts parallel and perpendicular to a normalized one-form and of the interplay of this decomposition with the geometric product. Section 3.6 explains the role played by the volume form and introduces the "twisted Hodge operator," a certain variant of the ordinary Hodge operator which is natural from the point of view of the Kähler-Atiyah algebra. Section 3.7 discusses the eigenvectors of the twisted Hodge operator, which we call "twisted (anti-)self-dual forms"; these will play a crucial role in later considerations. In Section 3.8, we recall the algebraic classification of the fiber type of the Clifford/KählerAtiyah bundle, which is an obvious application of the wellknown classification of Clifford algebras. We pay particular attention to the "nonsimple case"- the case when the fibers of the Kähler-Atiyah bundle fail to be simple as associative algebras over the base field. In Section 3.9, we discuss the spaces of twisted (anti-)self-dual forms in the nonsimple case, showing that-in this case-they form two-sided ideals of the Kähler-Atiyah algebra. We also give a description of such forms in terms of rank truncations, which is convenient in certain computations even though it is not well behaved with respect to the geometric product. In Section 3.10, we show that, in the presence of a globally defined one-form $\theta$ of unit norm, the spaces of twisted self-dual and twisted antiself-dual forms are isomorphic (as unital associative algebras !) with the space of those inhomogeneous forms which are orthogonal to $\theta-$ a space which always forms a subalgebra of the Kähler-Atiyah algebra. We also show that the components of an inhomogeneous form which are orthogonal and parallel to $\theta$ determine each other when the form is twisted (anti-)self-dual and give explicit formulas for the relation between these components in terms of what we call the "reduced twisted Hodge operator." Some of the material of this section is "well known" at least in certain circles, though the literature tends to be limited in its treatment of general dimensions and signatures and of certain other aspects. The reader who is familiar with geometric algebra may wish to concentrate on Sections 3.5, 3.6, 3.7, 3.9, and 3.10 and especially on our treatment of parallelism and orthogonality for twisted (anti-)self-dual forms, which is important for applications.

\subsection{Preparations: Wedge and Generalized Contraction Operators}

The Grading Automorphism. Let $\pi$ be that involutive $\mathscr{C}^{\infty}(M, \mathbb{K})$-linear automorphism of the exterior algebra 
$\left(\Omega_{\mathbb{K}}(M), \wedge\right)$ which is uniquely determined by the property that it acts as minus the identity on all one-forms. Thus

$$
\begin{aligned}
& \pi(\omega) \stackrel{\text { def }}{=} \sum_{k=0}^{d}(-1)^{k} \omega^{(k)}, \\
& \forall \omega=\sum_{k=0}^{d} \omega^{(k)} \in \Omega_{\mathbb{K}}(M), \text { where } \omega^{(k)} \in \Omega_{\mathbb{K}}^{k}(M) .
\end{aligned}
$$

Taking wedge products from the left and from the right with some inhomogeneous form $\omega \in \Omega_{\mathbb{K}}(M)$ defines $\mathscr{C}^{\infty}(M, \mathbb{R})$ linear operators $\wedge_{\omega}^{L}$ and $\wedge_{\omega}^{R}$ :

$$
\begin{aligned}
& \wedge_{\omega}^{L}(\eta)=\omega \wedge \eta, \\
& \wedge_{\omega}^{R}(\eta)=\eta \wedge \omega,
\end{aligned}
$$

$$
\forall \omega, \eta \in \Omega_{\mathbb{K}}(M)
$$

which satisfy the following identities by virtue of the fact that the wedge product is associative

$$
\begin{aligned}
& \wedge_{\omega_{1}}^{L} \circ \wedge_{\omega_{2}}^{L}=\Lambda_{\omega_{1} \wedge \omega_{2}}^{L}, \\
& \wedge_{\omega_{1}}^{R} \circ \wedge_{\omega_{2}}^{R}=\Lambda_{\omega_{2} \wedge \omega_{1}}^{R}, \\
& \wedge_{\omega_{1}}^{L} \circ \wedge_{\omega_{2}}^{R}=\Lambda_{\omega_{2}}^{R} \circ \Lambda_{\omega_{1}}^{L}, \\
& \forall \omega_{1}, \omega_{2} \in \Omega_{\mathbb{K}}(M)
\end{aligned}
$$

as well as the following relation, which encodes gradedcommutativity of the wedge product:

$$
\Lambda_{\omega}^{L}=\Lambda_{\omega}^{R} \circ \pi^{k} \Longleftrightarrow \Lambda_{\omega}^{R}=\Lambda_{\omega}^{L} \circ \pi^{k}, \quad \forall \omega \in \Omega_{\mathbb{K}}^{k}(M) .
$$

The Inner Product. Let $\langle$,$\rangle denote the symmetric nondegen-$ erate $\mathscr{C}^{\infty}(M, \mathbb{R})$-bilinear pairing (known as the inner product of inhomogeneous forms) induced by the metric $g$ on the exterior bundle. To be precise, this pairing is defined through

$$
\begin{aligned}
& \left\langle\alpha_{1} \wedge \cdots \wedge \alpha_{k}, \beta_{1} \wedge \cdots \wedge \beta_{l}\right\rangle \\
& \quad=\delta_{k l} \operatorname{det}\left(\hat{g}\left(\alpha_{i}, \beta_{j}\right)_{i, j=1 \cdots k}\right), \quad \forall \alpha_{i}, \beta_{j} \in \Omega_{\mathbb{K}}^{1}(M),
\end{aligned}
$$

a relation which fixes the convention used later in our computations (cf. Section 6) via the normalization property:

$$
\left\langle 1_{M}, 1_{M}\right\rangle=1
$$

Here, $\widehat{g}$ is the metric induced by $g$ on $T_{\mathbb{K}}^{*} M$, which gives the following pairing on one-forms $\alpha=\alpha_{a} e^{a}, \beta=\beta_{b} e^{b} \in \Omega_{\mathbb{K}}^{1}(M)$ :

$$
\widehat{g}(\alpha, \beta)=g^{a b} \alpha_{a} \beta_{b} \quad \text { for } \alpha=\alpha_{a} e^{a}, \beta=\beta_{b} e^{b} .
$$

The fixed rank components of $\Omega_{\mathbb{K}}(M)$ are mutually orthogonal with respect to the pairing $\langle$,$\rangle :$

$$
\langle\omega, \eta\rangle=0, \quad \forall \omega \in \Omega_{\mathbb{K}}^{k}(M), \forall \eta \in \Omega_{\mathbb{K}}^{l}(M), \forall k \neq l,
$$

so the rank decomposition $\Omega_{\mathbb{K}}(M)=\oplus_{k=0}^{d} \Omega_{\mathbb{K}}^{k}(M)$ is an orthogonal direct sum decomposition with respect to this pairing. Notice that the restriction of $\langle$,$\rangle to \Omega_{\mathbb{K}}^{1}(M)$ coincides with (11). Also notice that $\pi$ is self-adjoint with respect to the pairing $\langle$,$\rangle :$

$$
\langle\pi(\omega), \eta\rangle=\langle\omega, \pi(\eta)\rangle, \quad \forall \omega, \eta \in \Omega_{\mathbb{K}}(M) .
$$

Interior Products. The $\langle$,$\rangle -adjoints of the left and right wedge$ product operators (6) are denoted by $\iota_{\omega}^{R}$ and $\iota_{\omega}^{L}$ and are called the right and left generalized contraction (or interior product) operators, respectively:

$$
\begin{aligned}
& \left\langle\wedge_{\omega}^{L}(\eta), \rho\right\rangle=\left\langle\eta, \iota_{\omega}^{R}(\rho)\right\rangle, \\
& \left\langle\wedge_{\omega}^{R}(\eta), \rho\right\rangle=\left\langle\eta, \iota_{\omega}^{L}(\rho)\right\rangle,
\end{aligned}
$$

$$
\forall \omega, \eta, \rho \in \Omega_{\mathbb{K}}(M) .
$$

Properties (7) translate into

$$
\begin{aligned}
& \iota_{\omega_{1}}^{L} \circ \iota_{\omega_{2}}^{L}=\iota_{\omega_{1} \wedge \omega_{2}}^{L}, \\
& \iota_{\omega_{1}}^{R} \circ \iota_{\omega_{2}}^{R}=\iota_{\omega_{2} \wedge \omega_{1}}^{R}, \\
& \iota_{\omega_{1}}^{L} \circ \iota_{\omega_{2}}^{R}=\iota_{\omega_{2}}^{R} \circ l_{\omega_{1}}^{L},
\end{aligned}
$$

$$
\forall \omega_{1}, \omega_{2} \in \Omega_{\mathbb{K}}(M),
$$

while relation (8) is equivalent to

$$
\iota_{\omega}^{L}=\pi^{k} \circ \iota_{\omega}^{R} \Longleftrightarrow \iota_{\omega}^{R}=\pi^{k} \circ \iota_{\omega}^{L}, \quad \forall \omega \in \Omega_{\mathbb{K}}^{k}(M) .
$$

We also have $\wedge_{1_{M}}^{L, R}=\operatorname{id}_{\Omega_{\nwarrow}(M)}$ and $\iota_{1_{M}}^{L, R}=\operatorname{id}_{\Omega_{\nwarrow}(M)}$. Together with (7) and (15), this shows that $\wedge^{L}$ and $\wedge^{R}$ define a structure of $\left(\Omega_{\mathbb{K}}(M), \wedge\right)$-bimodule on $\Omega_{\mathbb{K}}(M)$ while $\iota^{L}$ and $\iota^{R}$ define another $\left(\Omega_{\mathbb{K}}(M), \wedge\right)$-bimodule structure on the same space. These two bimodule structures are adjoint to each other with respect to the pairing $\langle$,$\rangle .$

Identities (8) and (16) show that $\wedge_{\omega}^{L}$ and $\wedge_{\omega}^{R}$ determine each other while $\iota_{\omega}^{L}$ and $\iota_{\omega}^{R}$ also determine each other. From now on we choose to work with left wedge-multiplication

$$
\wedge_{\omega} \stackrel{\text { def }}{=} \wedge_{\omega}^{L}
$$

and with the following generalized contraction operator:

$$
\iota_{\omega}=\iota_{\tau(\omega)}^{R} \Longleftrightarrow\langle\omega \wedge \eta, \rho\rangle=\left\langle\eta, \iota_{\tau(\omega)}(\rho)\right\rangle,
$$

which satisfy

$$
\begin{gathered}
\wedge_{\omega_{1}} \circ \wedge_{\omega_{2}}=\wedge_{\omega_{1} \wedge \omega_{2}}, \\
\iota_{\omega_{1}} \circ l_{\omega_{2}}=\iota_{\omega_{1} \wedge \omega_{2}},
\end{gathered}
$$

$$
\forall \omega_{1}, \omega_{2} \in \Omega_{\mathbb{K}}(M)
$$

as well as

$$
\wedge_{1_{M}}=\iota_{1_{M}}=\mathrm{id}_{\Omega_{\nwarrow}(M)}
$$


and thus define two different structures of left module on the space $\Omega_{\mathbb{K}}(M)$ over the ring $\left(\Omega_{\mathbb{K}}(M), \wedge\right)$.

Wedge and Interior Product with a One-Form. For later reference, let us consider the case when $\theta \in \Omega_{\mathbb{K}}^{1}(M)$ is a one-form. Recall that the metric $g$ induces mutually inverse "musical isomorphisms" ${ }_{\sharp}: \Gamma\left(M, T_{\mathbb{K}} M\right) \rightarrow \Omega_{\mathbb{K}}^{1}(M)$ and ${ }^{\sharp}$ $\Omega_{\mathbb{K}}^{1}(M) \rightarrow \Gamma\left(M, T_{\mathbb{K}} M\right)$ defined by raising and lowering of indices, respectively:

$$
\begin{aligned}
& X=X^{a} e_{a} \Longrightarrow X_{\sharp}=X_{a} e^{a}, \quad \text { where } X_{a} \stackrel{\text { def }}{=} g_{a b} X^{b}, \\
& \theta=\theta_{a} e^{a} \Longrightarrow \theta^{\sharp}=\theta^{a} e_{a}, \quad \text { where } \theta^{a} \stackrel{\text { def }}{=} g^{a b} \theta_{b} .
\end{aligned}
$$

These isomorphisms satisfy

$$
\begin{aligned}
& g(X, Y)=\hat{g}\left(X_{\sharp}, Y_{\sharp}\right)=\left\langle X_{\sharp}, Y_{\sharp}\right\rangle, \\
& \forall X, Y \in \Gamma\left(M, T_{\mathbb{K}} M\right), \\
& g\left(\theta_{1}^{\sharp}, \theta_{2}^{\sharp}\right)=\hat{g}\left(\theta_{1}, \theta_{2}\right)=\left\langle\theta_{1}, \theta_{2}\right\rangle, \\
& \forall \theta_{1}, \theta_{2} \in \Omega_{\mathbb{K}}^{1}(M) .
\end{aligned}
$$

We have

$$
\begin{gathered}
X_{\sharp}=X_{\lrcorner} g, \\
\theta=\theta^{\sharp}{ }_{\lrcorner} g,
\end{gathered}
$$

where $X\lrcorner$ denotes the ordinary left contraction of a tensor with a vector field. It is not hard to see that the left contraction $\iota_{\theta}$ with a one-form coincides with the ordinary left contraction $\left.\theta^{\sharp}\right\lrcorner$ with the vector field $\theta^{\sharp}$ :

$$
\left.\iota_{\theta} \omega=\theta^{\sharp}\right\lrcorner \omega, \quad \forall \theta \in \Omega_{\mathbb{K}}^{1}(M), \forall \omega \in \Omega_{\mathbb{K}}(M) .
$$

Since $\theta \wedge \theta=0$, properties (19) imply ${ }^{3}$

$$
\wedge_{\theta} \circ \wedge_{\theta}=\iota_{\theta} \circ \iota_{\theta}=0 \text {. }
$$

Furthermore, the similar property of $\left.\theta^{\sharp}\right\lrcorner$ implies that $\iota_{\theta}$ is an odd derivation of the exterior algebra:

$$
\begin{aligned}
& \iota_{\theta}(\omega \wedge \eta)=\left(\iota_{\theta} \omega\right) \wedge \eta+\pi(\omega) \wedge \iota_{\theta} \eta, \\
& \forall \omega, \eta \in \Omega_{\mathbb{K}}(M) .
\end{aligned}
$$

Local Expressions. If $e_{a}$ is an arbitrary local frame of $M$ with dual coframe $e^{a}$ (thus $\left.e^{a}\left(e_{b}\right)=\delta_{b}^{a}\right)$, we let $g_{a b}=g\left(e_{a}, e_{b}\right)$ and $g^{a b}=\widehat{g}\left(e^{a}, e^{b}\right)$, so we have $g^{a b} g_{b c}=\delta_{c}^{a}$. The vector fields $\left(e^{a}\right)^{\sharp}$ satisfy $\left.\left(e^{a}\right)^{\sharp}\right\lrcorner g=e^{a}$ and are given explicitly by

$$
\left(e^{a}\right)^{\#}=g^{a b} e_{b} ;
$$

they form the contragradient local frame defined by $\left(e_{a}\right)$. We have $e_{a}=g_{a b}\left(e^{b}\right)^{\sharp}$ and $g\left(\left(e^{a}\right)^{\sharp},\left(e^{b}\right)^{\sharp}\right)=g^{a b}$. Thus

$$
\left.\left.\left.\iota_{e^{a}}=\left(e^{a}\right)^{\sharp}\right\lrcorner=g^{a b} e_{b}\right\lrcorner e_{a}\right\lrcorner=g_{a b} \iota_{e}^{b} .
$$

\subsection{Definition and First Properties of the} Kähler-Atiyah Algebra

The Geometric Product. Following an idea originally due to Chevalley and Riesz $[8,9]$, we identify $\mathrm{Cl}\left(T_{\mathbb{K}}^{*} M\right)$ with the exterior bundle $\wedge T_{\mathbb{K}}^{*} M$, thus realizing the Clifford product as the geometric product, which is the unique fiberwise associative, unital, and bilinear binary composition ${ }^{4} \diamond$ : $\wedge T_{\mathbb{K}}^{*} M \times_{M} \wedge T_{\mathbb{K}}^{*} M \rightarrow \wedge T_{\mathbb{K}}^{*} M$ whose induced action on sections (which we again denote by $\diamond$ ) satisfies the following relations for all $\theta \in \Omega_{\mathbb{K}}^{1}(M)$ and all $\omega \in \Omega_{\mathbb{K}}(M)$ :

$$
\begin{aligned}
\theta \diamond \omega & =\theta \wedge \omega+\iota_{\theta} \omega, \\
\pi(\omega) \diamond \theta & =\theta \wedge \omega-\iota_{\theta} \omega .
\end{aligned}
$$

Equations (29) determine the geometric composition of any two inhomogeneous forms via the requirement that the geometric product is associative and $\mathscr{C}^{\infty}(M, \mathbb{K})$-bilinear.

The unit of the fiber $\mathrm{Cl}\left(T_{\mathbb{K}, x}^{*} M\right)$ at a point $x \in M$ corresponds to the element $1 \in \mathbb{K}=\wedge^{0} T_{\mathbb{K}, x}^{*} M$, which is the unit of the associative algebra $\left(\wedge T_{\mathbb{K}, x}^{*} M, \diamond_{x}\right) \approx \mathrm{Cl}\left(T_{\mathbb{K}, x}^{*} M\right)$. Hence the unit section of the Clifford bundle $\mathrm{Cl}\left(T_{\mathbb{K}}^{*} M\right)$ is identified with the constant function $1_{M}: M \rightarrow \mathbb{K}$ given by $1_{M}(x)=1$ for all $x \in M$. Through this construction, the Clifford bundle is identified with the bundle of algebras $\left(\wedge T_{\mathbb{K}}^{*} M, \diamond\right)$, which is known [10] as the Kähler-Atiyah bundle of $(M, g)$. When endowed with the geometric product, the space $\Omega_{\mathbb{K}}(M)$ of all inhomogeneous $\mathbb{K}$-valued smooth forms on $M$ becomes a unital and associative (but noncommutative) algebra $\left(\Omega_{\mathbb{K}}(M), \diamond\right)$ over the ring $\mathscr{C}^{\infty}(M, \mathbb{R})$, known as the Kähler-Atiyah algebra of $(M, g)$. The unit of the KählerAtiyah algebra is the constant function $1_{M}$. We have a unital isomorphism of associative algebras over $\mathscr{C}^{\infty}(M, \mathbb{R})$ between $\left(\Omega_{\mathbb{K}}(M), \diamond\right)$ and the $\mathscr{C}^{\infty}(M, \mathbb{R})$-algebra $\Gamma\left(M, \mathrm{Cl}\left(T_{\mathbb{K}}^{*} M\right)\right)$ of all smooth sections of the Clifford bundle. The Kähler-Atiyah algebra can be viewed as a $\mathbb{Z}_{2}$-graded associative algebra with even and odd parts given by

$$
\begin{aligned}
& \Omega_{\mathbb{K}}^{\text {ev }}(M) \stackrel{\text { def }}{=} \oplus_{k=\text { even }} \Omega_{\mathbb{K}}^{k}(M), \\
& \Omega_{\mathbb{K}}^{\text {odd }}(M) \stackrel{\text { def }}{=} \oplus_{k=\text { odd }} \Omega_{\mathbb{K}}^{k}(M),
\end{aligned}
$$

since it is easy to check the inclusions:

$$
\begin{gathered}
\Omega_{\mathbb{K}}^{\text {ev }}(M) \diamond \Omega_{\mathbb{K}}^{\text {ev }}(M) \subset \Omega_{\mathbb{K}}^{\text {ev }}(M), \\
\Omega_{\mathbb{K}}^{\text {odd }}(M) \diamond \Omega_{\mathbb{K}}^{\text {odd }}(M) \subset \Omega_{\mathbb{K}}^{\text {ev }}(M), \\
\Omega_{\mathbb{K}}^{\text {ev }}(M) \diamond \Omega_{\mathbb{K}}^{\text {odd }}(M) \subset \Omega_{\mathbb{K}}^{\text {odd }}(M), \\
\Omega_{\mathbb{K}}^{\text {odd }}(M) \diamond \Omega_{\mathbb{K}}^{\text {ev }}(M) \subset \Omega_{\mathbb{K}}^{\text {odd }}(M) .
\end{gathered}
$$

However, it is not a $\mathbb{Z}$-graded algebra since the geometric product of two forms of definite rank need not be a form of definite rank. We let $P_{\mathrm{ev}}=(1 / 2)(1+\pi)$ and $P_{\mathrm{odd}}=$ $(1 / 2)(1-\pi)$ be the complementary idempotents associated with the decomposition into even and odd parts:

$$
\begin{gathered}
P_{\text {ev }}(\omega)=\omega_{\text {ev }} \\
P_{\text {odd }}(\omega)=\omega_{\text {odd }},
\end{gathered}
$$


where $\omega=\omega_{\text {ev }}+\omega_{\text {odd }} \in \Omega_{\mathbb{K}}(M)$, with $\omega_{\text {ev }} \in \Omega_{\mathbb{K}}^{\mathrm{ev}}(M)$ and $\omega_{\text {odd }} \in \Omega_{\mathbb{K}}^{\text {odd }}(M)$.

Generalized Products: Connection with Quantization of Spin Systems. The geometric product $\diamond$ can be viewed as a deformation of the wedge product (parameterized by the metric g) and reduces to the latter in the limit $g \rightarrow$ $\infty$; in this limit, the Kähler-Atiyah algebra reduces to the exterior algebra $\left(\Omega_{\mathbb{}}(M), \wedge\right)$. Under some mild assumptions, the geometric product can be described quite elegantly in the language of supermanifolds, as the star product induced by fiberwise Weyl quantization of a pure spin system [2023]. For this, consider the parity-changed tangent bundle $\Pi T M$ of $M$ (a supermanifold with body $M$ ) and introduce odd coordinates $\zeta^{a}$ on the fibers of $\Pi T U$, corresponding to a coframe $e^{a}$ of $M$ defined on a small enough open subset $U \subset M$. Inhomogeneous differential forms (2) correspond to functions defined on ПТМ having the following local expansion:

$$
f_{\omega}(x, \zeta)=_{U} \sum_{k=0}^{d} \frac{1}{k !} \omega_{a_{1} \cdots a_{k}}^{(k)}(x) \zeta^{a_{1}} \cdots \zeta^{a_{k}}
$$

This allows us to represent the geometric product through the fermionic analogue $\star$ of the Moyal product, using a certain "vertical" [23] quantization procedure

$$
f_{\omega} \star f_{\eta}=f_{\omega \diamond \eta}=f_{\omega} \exp \left(g^{a b} \frac{\check{\partial}}{\partial \zeta^{a}} \frac{\vec{\partial}}{\partial \zeta^{b}}\right) f_{\eta} .
$$

Expanding the exponential in (34) gives the following expressions for two general inhomogeneous forms $\omega, \eta \in \Omega_{\mathbb{}}(M)$ (cf. [24-26]):

$$
\begin{aligned}
\omega \diamond \eta= & \sum_{k=0}^{[d / 2]} \frac{(-1)^{k}}{(2 k) !} \omega \wedge_{2 k} \eta \\
& +\sum_{k=0}^{[(d-1) / 2]} \frac{(-1)^{k+1}}{(2 k+1) !} \pi(\omega) \wedge_{2 k+1} \eta,
\end{aligned}
$$

where the binary $\mathscr{C}^{\infty}(M, \mathbb{R})$-bilinear operations $\wedge_{k}$ are the contracted wedge products [24-27], defined iteratively through

$$
\begin{aligned}
\omega \wedge_{0} \eta & =\omega \wedge \eta, \\
\omega \wedge_{k+1} \eta & \left.=g^{a b}\left(e_{a\lrcorner} \omega\right) \wedge_{k}\left(e_{b\lrcorner}\right\lrcorner\right)=g_{a b}\left(\iota_{e^{a}} \omega\right) \wedge_{k}\left(\iota_{e^{b}} \eta\right) .
\end{aligned}
$$

We also have the following expansions for the graded $\diamond$ commutator and graded $\diamond$-anticommutator of $\omega$ with $\eta$ :

$$
\begin{aligned}
& {[[\omega, \eta]]_{-, \diamond}=2 \sum_{k=0}^{[(d-1) / 2]} \frac{(-1)^{k+1}}{(2 k+1) !} \pi(\omega) \wedge_{2 k+1} \eta} \\
& {[[\omega, \eta]]_{+, \diamond}=2 \sum_{k=0}^{[d / 2]} \frac{(-1)^{k}}{(2 k) !} \omega \wedge_{2 k} \eta .}
\end{aligned}
$$

For forms of definite ranks, the graded $\diamond$-commutator and graded $\diamond$-anticommutator are of course defined through

$$
\begin{aligned}
{[[\omega, \eta]]_{-, \diamond} \stackrel{\text { def }}{=} \omega \diamond \eta-(-1)^{p q} \eta \diamond \omega, } \\
{[[\omega, \eta]]_{+, \diamond} \stackrel{\text { def }}{=} \omega \diamond \eta+(-1)^{p q} \eta \diamond \omega, } \\
\\
\quad \forall \omega \in \Omega_{\mathbb{K}}^{p}(M), \forall \eta \in \Omega_{\mathbb{K}}^{q}(M),
\end{aligned}
$$

being extended by linearity to the entire space $\Omega_{\mathbb{K}}(M)$. Using (35) one can easily deduce the following relation for homogeneous forms:

$$
\begin{aligned}
\eta \diamond \omega=(-1)^{p q} & \sum_{k=0}^{p} \frac{(-1)^{k(p-k+1)+[k / 2]}}{k !} \omega \wedge_{k} \eta, \\
& \forall \omega \in \Omega_{\mathbb{K}}^{p}(M), \quad \forall \eta \in \Omega_{\mathbb{K}}^{q}(M), \quad p \leq q .
\end{aligned}
$$

We will mostly use, instead of $\wedge_{k}$, the so-called generalized products $\triangle_{k}$, which are defined by rescaling the contracted wedge products:

$$
\triangle_{k}=\frac{1}{k !} \wedge_{k} .
$$

These have the advantage that the various factorial prefactors in the expansions above disappear when those expansions are reexpressed in terms of generalized products.

Expansions (35) and (37) can also be obtained directly from the definition of the geometric product using (29), which shows that the purely mathematical identities given above also hold irrespective of any interpretation through the theory of quantization of spin systems.

3.3. (Anti-)Automorphisms of the Kähler-Atiyah Algebra. Direct computation shows that $\pi$ is an involutive automorphism (known as the main or grading automorphism) of the Kähler-Atiyah bundle (a property which, in the limit $g \rightarrow \infty$, recovers the well-known fact that $\pi$ is also an automorphism of the exterior bundle). The Kähler-Atiyah bundle also admits an involutive antiautomorphism $\tau$ (known as the main antiautomorphism or as reversion), which is given by

$$
\tau(\omega) \stackrel{\text { def }}{=}(-1)^{k(k-1) / 2} \omega, \quad \forall \omega \in \Omega_{\mathbb{K}}^{k}(M) .
$$

It is the unique antiautomorphism of $\left(\wedge T_{\mathbb{K}}^{*} M, \diamond\right)$ which acts trivially on all one-forms (i.e., which satisfies $\tau(\theta)=\theta$ for any form $\theta$ of rank one). Direct computation (or the fact that the exterior product is recovered from the diamond product in the limit of infinite metric) shows that $\tau$ is also an antiautomorphism of the exterior bundle $\left(T_{\mathbb{K}}^{*} M, \wedge\right)$. We also notice that $\pi$ and $\tau$ commute. All in all, we have the relations:

$$
\begin{aligned}
& \pi \circ \tau=\tau \circ \pi, \\
& \pi \circ \pi=\tau \circ \tau=\operatorname{id}_{\Omega_{K}(M)} .
\end{aligned}
$$

Note that $\mathrm{Cl}^{\mathrm{ev}}\left(T_{\mathbb{K}}^{*} M\right)$ identifies with the subbundle of unital subalgebras $\wedge^{\mathrm{ev}} T_{\mathbb{K}}^{*} M=\oplus_{k=\text { even }} \wedge{ }^{k} T_{\mathbb{K}}^{*} M$ of the KählerAtiyah bundle, whose space of smooth sections $\Omega_{\mathbb{K}}^{\mathrm{ev}}(M)$ can 
be described as the eigenspace of $\pi$ corresponding to the eigenvalue +1 :

$$
\Omega_{\mathbb{K}}^{\mathrm{ev}}(M)=\mathscr{K}(1-\pi)=\left\{\omega \in \Omega_{\mathbb{K}}(M) \mid \pi(\omega)=\omega\right\} .
$$

3.4. The Left and Right Geometric Multiplication Operators. Let $L_{\omega}, R_{\omega}$ be the $\mathscr{C}^{\infty}(M, \mathbb{R})$-linear operators of left and right multiplication with $\omega \in \Omega_{\mathbb{K}}(M)$ in the Kähler-Atiyah algebra:

$$
\begin{aligned}
& L_{\omega}(\eta) \stackrel{\text { def }}{=} \omega \diamond \eta, \\
& R_{\omega}(\eta) \stackrel{\text { def }}{=} \eta \diamond \omega, \\
& \qquad \omega, \eta \in \Omega_{\mathbb{K}}(M) .
\end{aligned}
$$

These satisfy

$$
\begin{aligned}
& L_{\omega_{1}} \circ R_{\omega_{2}}=R_{\omega_{2}} \circ L_{\omega_{1}}, \\
& L_{\omega_{1}} \circ L_{\omega_{2}}=L_{\omega_{1} \diamond \omega_{2}}, \\
& R_{\omega_{1}} \circ R_{\omega_{2}}=R_{\omega_{2} \diamond \omega_{1}}
\end{aligned}
$$

as a consequence of associativity of the geometric product. We also have

$$
\begin{aligned}
& L_{\omega} \circ \pi=\pi \circ L_{\pi(\omega)}, \\
& R_{\omega} \circ \pi=\pi \circ R_{\pi(\omega)}, \\
& L_{\omega} \circ \tau=\tau \circ R_{\tau(\omega)}, \\
& \tau \circ L_{\omega}=R_{\tau(\omega)} \circ \tau,
\end{aligned}
$$

$$
\forall \omega \in \Omega_{\mathbb{K}}(M),
$$

since $\pi$ is an involutive algebra automorphism while $\tau$ is an involutive antiautomorphism. Identity (29) can be written as

$$
\begin{aligned}
L_{\theta} & =\wedge_{\theta}+\iota_{\theta}, \\
R_{\theta} \circ \pi & =\wedge_{\theta}-\iota_{\theta},
\end{aligned}
$$

being equivalent to

$$
\forall \theta \in \Omega_{\mathbb{K}}^{1}(M),
$$

$$
\begin{aligned}
\wedge_{\theta} & =\frac{1}{2}\left(L_{\theta}+R_{\theta} \circ \pi\right), \\
\iota_{\theta} & =\frac{1}{2}\left(L_{\theta}-R_{\theta} \circ \pi\right),
\end{aligned}
$$

$$
\forall \theta \in \Omega_{\mathbb{K}}^{1}(M) .
$$

This shows that the operators $\wedge_{\theta}$ and $\iota_{\theta}$ (and thus-given properties (19) —also the operators $\wedge_{\omega}$ and $\iota_{\omega}$ for any $\omega \epsilon$ $\left.\Omega_{\mathbb{K}}(M)\right)$ are determined by the geometric product.

Remark 4. Equation (29) implies

$$
\begin{aligned}
\iota_{\theta} \omega & =\frac{1}{2}[[\theta, \omega]]_{-, \diamond}, \\
\theta \wedge \omega & =\frac{1}{2}[[\theta, \omega]]_{+, \diamond},
\end{aligned}
$$

The first identity in (49) shows that the operator $\iota_{\theta}$ is an odd $\mathscr{C}^{\infty}(M, \mathbb{R})$-linear derivation (in fact, an odd differentialsince $\left.\iota_{\theta} \circ \iota_{\theta}=0\right)$ of the Kähler-Atiyah algebra:

$$
\begin{aligned}
\iota_{\theta}(\omega \diamond \eta)=\iota_{\theta}(\omega) \diamond \eta+\pi(\omega) \diamond \iota_{\theta}(\eta), & \\
& \forall \omega, \eta \in \Omega_{\mathbb{K}}(M), \forall \theta \in \Omega^{1}(M) .
\end{aligned}
$$

In the limit $g \rightarrow \infty$, this property recovers (26). Notice that $\wedge_{\theta}$ is not a derivation of the Kähler-Atiyah algebra; however, it satisfies $\wedge_{\theta} \circ \wedge_{\theta}=0$.

3.5. Orthogonality and Parallelism. Let $\theta \in \Omega_{\mathbb{K}}^{1}(M)$ be a fixed one-form which satisfies the normalization condition:

$$
\widehat{g}(\theta, \theta)=1 \text { that is } \iota_{\theta} \theta=1 \text {. }
$$

This condition is equivalent to

$$
\theta \diamond \theta=1,
$$

a fact which follows from (29) and from the identity $\theta \wedge \theta=0$ (which, together, imply $\theta \diamond \theta=\iota_{\theta} \theta$ ).

We say that an inhomogeneous form $\omega \in \Omega_{\mathbb{K}}(M)$ is parallel to $\theta$ (we write $\theta \| \omega$ ) if $\theta \wedge \omega=0$ and orthogonal to $\theta$ (we write $\theta \perp \omega$ ) if $\iota_{\theta} \omega=0$. Thus

$$
\begin{aligned}
\theta \| \omega \stackrel{\text { def }}{\Longleftrightarrow} \omega \in \mathscr{K}\left(\wedge_{\theta}\right), \\
\theta \perp \omega \stackrel{\text { def }}{\Longleftrightarrow} \omega \in \mathscr{K}\left(\iota_{\theta}\right),
\end{aligned}
$$

where we remind the reader that $\mathscr{K}(A)$ denotes the kernel of any $\mathbb{K}$-linear operator $A: \Omega_{\mathbb{K}}(M) \rightarrow \Omega_{\mathbb{K}}(M)$. Properties (25) imply $\mathscr{I}\left(\iota_{\theta}\right) \subset \mathscr{K}\left(\iota_{\theta}\right)$ and $\mathscr{I}\left(\wedge_{\theta}\right) \subset \mathscr{K}\left(\wedge_{\theta}\right)$, where $\mathscr{I}(A)$ denotes the image of any $\mathbb{K}$-linear operator $A: \Omega_{\mathbb{K}}(M) \rightarrow$ $\Omega_{\mathbb{K}}(M)$. These inclusions are in fact equalities, as we will see in a moment.

Proposition 5. Any inhomogeneous differential form $\omega \in$ $\Omega_{\mathbb{K}}(M)$ decomposes uniquely as

$$
\omega=\omega_{\|}+\omega_{\perp},
$$

where $\theta \quad \| \quad \omega_{\|}$and $\theta \perp \omega_{\perp}$. Moreover, the parallel and orthogonal parts of $\omega$ are given by

$$
\begin{gathered}
\omega_{\|}=\theta \wedge\left(\iota_{\theta} \omega\right), \\
\omega_{\perp}=\iota_{\theta}(\theta \wedge \omega) .
\end{gathered}
$$

In fact, the $\mathscr{C}^{\infty}(M, \mathbb{R})$-linear operators $P_{\|} \stackrel{\text { def }}{=} \wedge_{\theta} \circ \iota_{\theta}$ and $P_{\perp} \stackrel{\text { def }}{=}$ $\iota_{\theta} \circ \wedge_{\theta}$ are complementary idempotents:

$$
\begin{aligned}
P_{\|}+P_{\perp} & =\mathrm{id}_{\Omega_{\aleph}(M)}, \\
P_{\|} \circ P_{\|} & =P_{\|}, \\
P_{\perp} \circ P_{\perp} & =P_{\perp}, \\
P_{\|} \circ P_{\perp} & =P_{\perp} \circ P_{\|}=0 .
\end{aligned}
$$


Proof. The statements of the proposition follow immediately from the fact that $\wedge_{\theta}$ and $\iota_{\theta}$ are nilpotent and because $\iota_{\theta}$ is an odd derivation of the wedge product, which implies

$$
\iota_{\theta}(\theta \wedge \omega)=\omega-\theta \wedge\left(\iota_{\theta} \omega\right)
$$

where we used the normalization condition (51).
As an immediate corollary of the proposition, we find the well-known equalities

$$
\begin{aligned}
& \mathscr{K}\left(\iota_{\theta}\right)=\mathscr{I}\left(\iota_{\theta}\right), \\
& \mathscr{K}\left(\wedge_{\theta}\right)=\mathscr{I}\left(\wedge_{\theta}\right)
\end{aligned}
$$

as well as the characterizations:

$$
\begin{gathered}
\theta \| \omega \Longleftrightarrow \omega=\theta \wedge \alpha \quad \text { with } \alpha \in \Omega_{\mathbb{K}}(M) \Longleftrightarrow \theta \diamond \omega=-\pi(\omega) \diamond \theta \Longleftrightarrow \omega \in \mathscr{K}\left(L_{\theta}+R_{\theta} \circ \pi\right), \\
\theta \perp \omega \Longleftrightarrow \omega=\iota_{\theta} \beta \quad \text { with } \beta \in \Omega_{\mathbb{K}}(M) \Longleftrightarrow \theta \diamond \omega=\pi(\omega) \diamond \theta \Longleftrightarrow \omega \in \mathscr{K}\left(L_{\theta}-R_{\theta} \circ \pi\right),
\end{gathered}
$$

where we used relations (48). Thus $\theta \| \omega$ iff $\omega$ graded anticommutes with $\theta$ and $\theta \perp \omega$ iff $\omega$ graded commutes with $\theta$ in the Kähler-Atiyah algebra.

Behavior with respect to the Geometric Product. Consider the following $\mathscr{C}^{\infty}(M, \mathbb{R})$-submodules of $\Omega_{\mathbb{K}}(M)$ :

$$
\begin{aligned}
& \Omega_{\mathbb{K}}^{\|}(M) \stackrel{\text { def }}{=}\left\{\omega \in \Omega_{\mathbb{K}}(M) \mid \theta \| \omega\right\}, \\
& \Omega_{\mathbb{K}}^{\perp}(M) \stackrel{\text { def }}{=}\left\{\omega \in \Omega_{\mathbb{K}}(M) \mid \theta \perp \omega\right\} .
\end{aligned}
$$

Using the characterizations in (59), we find

$$
\begin{gathered}
\theta\|\omega, \theta \perp \eta \Longrightarrow \theta\|(\omega \diamond \eta), \theta \|(\eta \diamond \omega), \\
\theta \| \omega, \eta \Longrightarrow \theta \perp(\omega \diamond \eta), \\
\theta \perp \omega, \eta \Longrightarrow \theta \perp(\omega \diamond \eta),
\end{gathered}
$$

which translate into

$$
\begin{aligned}
& (\omega \diamond \eta)_{\|}=\omega_{\|} \diamond \eta_{\perp}+\omega_{\perp} \diamond \eta_{\|}, \\
& (\omega \diamond \eta)_{\perp}=\omega_{\|} \diamond \eta_{\|}+\omega_{\perp} \diamond \eta_{\perp} .
\end{aligned}
$$

We thus have the inclusions:

$$
\begin{aligned}
& \Omega_{\mathbb{K}}^{\|}(M) \diamond \Omega_{\mathbb{K}}^{\|}(M) \subset \Omega_{\mathbb{K}}^{\perp}(M), \\
& \Omega_{\mathbb{K}}^{\perp}(M) \diamond \Omega_{\mathbb{K}}^{\perp}(M) \subset \Omega_{\mathbb{K}}^{\perp}(M), \\
& \Omega_{\mathbb{K}}^{\|}(M) \diamond \Omega_{\mathbb{K}}^{\perp}(M) \subset \Omega_{\mathbb{K}}^{\|}(M), \\
& \Omega_{\mathbb{K}}^{\perp}(M) \diamond \Omega_{\mathbb{K}}^{\|}(M) \subset \Omega_{\mathbb{K}}^{\|}(M) .
\end{aligned}
$$

Together with the identity $\iota_{\theta}\left(1_{M}\right)=0$ (which shows that $\theta \perp$ $1_{M}$ ), the last property in (61) shows that $\Omega_{\mathbb{K}}^{\perp}(M)$ is a unital subalgebra of the Kähler-Atiyah algebra.

Notice that characterizations (59) imply that the involutions $\pi$ and $\tau$ preserve parallelism and orthogonality to $\theta$ :

$$
\begin{aligned}
\theta \| \omega & \Longrightarrow \theta \| \pi(\omega), \\
\theta & \perp \omega \Longrightarrow \theta \perp \pi(\omega), \\
\theta \| \omega & \Longrightarrow \theta \| \tau(\omega), \\
\theta & \perp \omega \Longrightarrow \theta \perp \tau(\omega) .
\end{aligned}
$$

The Top Component of an Inhomogeneous Form. The parallel part of $\omega \in \Omega_{\mathbb{K}}(M)$ can be written as

$$
\omega_{\|}=\theta \wedge \omega_{\top},
$$

where

$$
\omega_{\top} \stackrel{\text { def }}{=} \iota_{\theta} \omega \in \Omega_{\mathbb{K}}^{\perp}(M) .
$$

This shows that $\omega$ determines and is determined by the two inhomogeneous forms $\omega_{\perp}$ and $\omega_{\top}$, both of which belong to $\Omega_{\mathbb{K}}^{\perp}(M)$. In fact, any $\omega \in \Omega_{\mathbb{K}}(M)$ can be written uniquely in the form

$$
\omega=\theta \wedge \alpha+\beta \quad \text { with } \alpha, \beta \in \Omega_{\mathbb{K}}^{\perp}(M) ;
$$

namely, we have $\alpha=\omega_{\mathrm{T}}$ and $\beta=\omega_{\perp}$. This gives a $\mathscr{C}^{\infty}(M, \mathbb{R})$ linear isomorphism:

$$
\Omega_{\mathbb{K}}(M) \stackrel{\iota_{\theta}+P_{\perp}}{\longrightarrow} \Omega^{\perp}(M) \oplus \Omega^{\perp}(M) .
$$

which sends $\omega \in \Omega_{\mathbb{K}}(M)$ into the pair $\left(\omega_{\top}, \omega_{\perp}\right)$ and whose inverse sends a pair $(\alpha, \beta)$ with $\alpha, \beta \in \Omega_{\mathbb{K}}^{\perp}(M)$ into the form (67). Since $\omega_{T}$ is orthogonal to $\theta$, we have $\theta \wedge \omega_{T}=\theta \diamond$ $\omega_{\top}=\pi\left(\omega_{T}\right) \diamond \theta$ and thus $\omega_{\|}=\theta \diamond \omega_{T}$. It follows that the decomposition of $\omega$ can be written entirely in terms of the geometric product:

$$
\omega=\theta \diamond \omega_{\top}+\omega_{\perp} .
$$

An easy computation using this formula gives

$$
\begin{aligned}
& (\omega \diamond \eta)_{\perp}=\omega_{\perp} \diamond \eta_{\perp}+\pi\left(\omega_{\top}\right) \diamond \eta_{\top}, \\
& (\omega \diamond \eta)_{\top}=\omega_{\top} \diamond \eta_{\perp}+\pi\left(\omega_{\perp}\right) \diamond \eta_{\top} .
\end{aligned}
$$

\subsection{The Volume Form and the Twisted Hodge Duality Operator}

The Ordinary Volume Form. From now on, we will assume that $M$ is oriented (in particular, the $\mathbb{K}$-line bundles $\Lambda^{d} T_{\mathbb{K}}^{*} M$ are trivial for $\mathbb{K}=\mathbb{R}, \mathbb{C}$ ). Consider the volume form determined on $M$ by the metric and by this orientation, which has the following expression in a local frame defined on $U \subset$ M:

$$
\operatorname{vol}_{M}=\frac{1}{d !} \sqrt{|\operatorname{det} g|} \epsilon_{a_{1} \cdots a_{d}} e^{a_{1} \cdots a_{d}}
$$


Here, det $g$ is the determinant of the matrix $\left(g\left(e_{a}, e_{b}\right)\right)_{a, b=1 \cdots d}$ while $\epsilon_{a_{1} \cdots a_{d}}$ are the local coefficients of the Ricci densitydefined as the signature of the permutation $\left(\begin{array}{cccc}1 & 2 & \cdots & d \\ a_{1} & a_{2} & \cdots & a_{d}\end{array}\right)$. The volume form satisfies

$$
\begin{aligned}
\operatorname{vol}_{M} \diamond \operatorname{vol}_{M}=(-1)^{q+d(d-1) / 2}=(-1)^{q+[d / 2]} \\
\quad= \begin{cases}+1, & \text { if } p-q \equiv_{4} 0,1 \Longleftrightarrow p-q \equiv_{8} 0,1,4,5 \\
-1, & \text { if } p-q \equiv_{4} 2,3 \Longleftrightarrow p-q \equiv_{8} 2,3,6,7,\end{cases}
\end{aligned}
$$

where we used the congruences:

$$
\begin{gathered}
\frac{d(d-1)}{2} \equiv_{2}\left[\frac{d}{2}\right], \\
q+\frac{d(d-1)}{2} \equiv_{2} \begin{cases}\frac{p-q}{2}, & \text { if } d=\text { even } \\
\frac{p-q-1}{2}, & \text { if } d=\text { odd. }\end{cases}
\end{gathered}
$$

We remind the reader that $p$ and $q$ denote the number of positive and negative eigenvalues of the metric tensor, respectively.

The Ordinary Hodge Operator. Recall that the ordinary $\mathscr{C}^{\infty}(M, \mathbb{R})$-linear Hodge operator $*$ is defined through

$$
\begin{aligned}
\omega \wedge(* \eta)=\langle\omega, \eta\rangle \operatorname{vol}_{M} & \\
& \forall \omega, \eta \in \Omega_{\mathbb{K}}^{k}(M), \forall k=0 \cdots d
\end{aligned}
$$

and satisfies the following properties, which we list for convenience of the reader:

$$
\begin{aligned}
\omega \wedge \eta & =(-1)^{q}\langle\eta, * \omega\rangle \operatorname{vol}_{M}, \\
\forall \omega & \in \Omega^{k}(M), \forall \eta \in \Omega^{d-k}(M), \forall k=0 \cdots d, \\
\langle * \omega, * \eta\rangle & =(-1)^{q}\langle\omega, \eta\rangle, \quad \forall \omega, \eta \in \Omega(M), \\
\operatorname{vol}_{M} & =* 1_{M} \Longleftrightarrow \operatorname{vol}_{M}=(-1)^{q} 1_{M}, \\
* \omega & =\iota_{\omega} \operatorname{vol}_{M}, \quad \forall \omega \in \Omega(M), \\
* \circ * & =(-1)^{q} \pi^{d-1}, \\
* \circ \pi & =(-1)^{d} \pi \circ * .
\end{aligned}
$$

We also note the identity

$$
\tau \circ *=(-1)^{[d / 2]} * \circ \tau \circ \pi^{d-1}
$$

which follows by direct computation upon using the congruence:

$$
\begin{gathered}
\frac{k(k-1)}{2}+\frac{(d-k)(d-k-1)}{2} \\
\equiv_{2} \frac{d(d-1)}{2}+k(d-1) .
\end{gathered}
$$

The Modified Volume Form. Consider the following $\mathbb{K}$-valued top form on $M$ :

$$
\nu \stackrel{\text { def }}{=} c_{p, q}(\mathbb{K}) \operatorname{vol}_{M} \text {, }
$$

$$
\text { where } c_{p, q}(\mathbb{K}) \stackrel{\text { def }}{=} \begin{cases}1, & \text { if } \mathbb{K}=\mathbb{R} \\ i^{q+[d / 2]}, & \text { if } \mathbb{K}=\mathbb{C},\end{cases}
$$

which satisfies

$$
\nu \diamond \nu= \begin{cases}(-1)^{q+[d / 2]} 1_{M}, & \text { if } \mathbb{K}=\mathbb{R} \\ +1_{M}, & \text { if } \mathbb{K}=\mathbb{C} .\end{cases}
$$

We have the normalization property

$$
\langle\nu, v\rangle= \begin{cases}(-1)^{q} 1_{M}, & \text { if } \mathbb{K}=\mathbb{R} \\ (-1)^{[d / 2]} 1_{M}, & \text { if } \mathbb{K}=\mathbb{C}\end{cases}
$$

and the identity

$$
\begin{aligned}
L_{\nu}=R_{\nu} \circ \pi^{d-1} \Longleftrightarrow \nu \diamond \omega=\pi^{d-1}(\omega) & \diamond \nu, \\
& \forall \omega \in \Omega_{\mathbb{K}}(M),
\end{aligned}
$$

where $\pi^{d-1}$ represents the composition of $d-1$ copies of the main automorphism $\pi$ :

$$
\pi^{d-1}= \begin{cases}\operatorname{id}_{\Omega_{\mathbb{}}(M)}, & \text { if } d=\text { odd } \\ \pi, & \text { if } d=\text { even. }\end{cases}
$$

In particular, $v$ is a central element of the Kähler-Atiyah algebra iff $d$ is odd.

The Twisted Hodge Operator. Let us define the $\left(\mathscr{C}^{\infty}(M, \mathbb{R})\right.$ linear) twisted Hodge operator $\widetilde{*}: \Omega_{\mathbb{K}}(M) \rightarrow \Omega_{\mathbb{K}}(M)$ through the formula:

$$
\widetilde{*} \omega \stackrel{\text { def }}{=} \omega \diamond \nu, \quad \forall \omega \in \Omega_{\mathbb{K}}(M) .
$$

Identity (79) shows that (unlike what happens for the ordinary Hodge operator) the square of the twisted Hodge operator is always a scalar multiple of the identity:

$$
\widetilde{*} \circ \widetilde{*}= \begin{cases}(-1)^{q+[d / 2]} \mathrm{id}_{\Omega_{\mathbb{K}}(M)}, & \text { if } \mathbb{K}=\mathbb{R} \\ \operatorname{id}_{\Omega_{\mathbb{K}}(M)}, & \text { if } \mathbb{K}=\mathbb{C} .\end{cases}
$$

A simple computation shows that the twisted and ordinary Hodge operators are related through

$$
\widetilde{*}=c_{p, q}(\mathbb{K}) * \circ \tau .
$$

In particular, the ordinary Hodge operator admits the representation:

$$
* \omega=\tau(\omega) \diamond \operatorname{vol}_{M}=\frac{1}{c_{p, q}(\mathbb{K})} \tau(\omega) \diamond \nu,
$$


3.7. Twisted (Anti-)Self-Dual Forms. Let us assume that $\mathbb{K}=$ $\mathbb{C}$ or that $\mathbb{K}=\mathbb{R}$ and $p-q \equiv_{4} 0,1$, so that the twisted Hodge operator $\widetilde{*}$ squares to the identity. In this case, the twisted Hodge operator has real eigenvalues equal to \pm 1 and we can consider inhomogeneous real forms belonging to the corresponding eigenspaces. A form $\omega \in \Omega_{\mathbb{K}}(M)$ will be called twisted self-dual if $\widetilde{* \omega}=+\omega$ and twisted anti-self-dual if $\widetilde{*} \omega=-\omega$. We let $\Omega_{\mathbb{K}}^{ \pm}(M) \stackrel{\text { def }}{=}\left\{\omega \in \Omega_{\mathbb{K}}(M) \mid \omega \diamond \nu=\right.$ $\pm \omega\} \subset \Omega_{\mathbb{K}}(M)$ be the $\mathscr{C}^{\infty}(M, \mathbb{R})$-submodules of twisted selfdual and twisted anti-self-dual forms on $M$.

The Ideals $\Omega_{\mathbb{K}}^{ \pm}(M)$. The elements $p_{ \pm} \stackrel{\text { def }}{=}(1 / 2)(1 \pm v)$ are complementary idempotents of the Kähler-Atiyah algebra:

$$
\begin{aligned}
& p_{ \pm} \circ p_{ \pm}=p_{ \pm}, \\
& p_{+}+p_{-}=1_{M}, \\
& p_{ \pm} \circ p_{\mp}=0 .
\end{aligned}
$$

Notice that these idempotents are central only when $v$ is central, that is, only when $d$ is odd. The operators $P_{ \pm} \stackrel{\text { def }}{=} R_{p_{ \pm}}$ defined through right $\diamond$-multiplication with these elements

$$
\begin{aligned}
P_{ \pm}(\omega) \stackrel{\text { def }}{=} \omega \diamond p_{ \pm}= & \frac{1}{2}(\omega \pm \omega \diamond \nu) \\
& \left(\omega \in \Omega_{\mathbb{K}}(M)\right) \Longleftrightarrow P_{ \pm}=\frac{1}{2}(1 \pm \widetilde{*})
\end{aligned}
$$

are complementary idempotents in the algebra of endomorphisms of the $\mathscr{C}^{\infty}(M, \mathbb{R})$-module $\Omega_{\mathbb{K}}(M)$ :

$$
\begin{gathered}
P_{ \pm}^{2}=P_{ \pm}, \\
P_{+} \circ P_{-}=P_{-} \circ P_{+}=0, \\
P_{+}+P_{-}=\mathrm{id}_{\Omega_{\nwarrow}(M)} .
\end{gathered}
$$

Therefore, the images $\Omega_{\mathbb{K}}^{ \pm}(M)=P_{ \pm}\left(\Omega_{\mathbb{K}}(M)\right)=\Omega_{\mathbb{K}}(M) p_{ \pm}$ are complementary left ideals of the Kähler-Atiyah algebra, giving the direct sum decomposition:

$$
\Omega_{\mathbb{K}}(M)=\Omega_{\mathbb{K}}^{+}(M) \oplus \Omega_{\mathbb{K}}^{-}(M) .
$$

In particular, $\left(\Omega_{\mathbb{K}}^{ \pm}(M), \diamond\right)$ are associative subalgebras of the Kähler-Atiyah algebra. These subalgebras have units (given by $\left.p_{ \pm}\right)$iff $d$ is odd, in which case they are two-sided ideals of $\left(\Omega_{\mathbb{K}}(M), \diamond\right)$.

Local Characterization. With respect to a local coframe $e^{a}$ above an open subset $U \subset M$, we have the expansions:

$$
\begin{aligned}
& *\left(e^{a_{1} \cdots a_{k}}\right)=\frac{1}{(d-k) !} \sqrt{|\operatorname{det} g|} \epsilon^{a_{1} \cdots a_{k}} a_{k+1} \cdots a_{d} e^{a_{k+1} \cdots a_{d}}, \\
& \widetilde{*}\left(e^{a_{1} \cdots a_{k}}\right) \\
& =\frac{1}{(d-k) !} c_{p, q}(\mathbb{K}) \sqrt{|\operatorname{det} g|} \epsilon^{a_{k} \cdots a_{1}} a_{k+1} \cdots a_{d} e^{a_{k+1} \cdots a_{d}},
\end{aligned}
$$

where indices are raised with $g^{a b}$. Using (91), one easily checks that an inhomogeneous form $\omega \in \Omega_{\mathbb{K}}(M)$ with expansion (2) satisfies $\widetilde{*} \omega= \pm \omega$ iff its nonstrict coefficients satisfy the conditions:

$$
\begin{aligned}
& \omega_{a_{1} \cdots a_{k}}^{(k)} \\
& = \pm \frac{(-1)^{k(d-k)}}{(d-k) !} c_{p, q}(\mathbb{K}) \sqrt{|\operatorname{det} g|} \epsilon_{a_{1} \cdots a_{k}} a_{d} \cdots a_{k+1} \omega_{a_{k+1} \cdots a_{d}}^{(d-k)}, \\
& \forall k=0, \ldots, d .
\end{aligned}
$$

We note here for future reference the expansions for the Hodge dual and the twisted Hodge dual of any $k$-form $\omega$ :

$$
\begin{aligned}
& (* \omega)_{a_{k+1} \cdots a_{d}}=\frac{(-1)^{k(d-k)}}{(d-k) !} \sqrt{|\operatorname{det} g|} \epsilon_{a_{k+1} \cdots a_{d}}{ }^{a_{1} \cdots a_{k}} \omega_{a_{1} \cdots a_{k}}, \\
& (\widetilde{*} \omega)_{a_{k+1} \cdots a_{d}} \\
& =\frac{(-1)^{k(d-k)}}{(d-k) !} c_{p, q}(\mathbb{K}) \sqrt{|\operatorname{det} g|} \epsilon_{a_{d} \cdots a_{k+1}}{ }^{a_{1} \cdots a_{k}} \omega_{a_{1} \cdots a_{k}} .
\end{aligned}
$$

3.8. Algebraic Classification of Fiber Types. The fibers of the Kähler-Atiyah bundle are isomorphic with the Clifford algebra $\mathrm{Cl}_{\mathbb{K}}(p, q)=\mathrm{Cl}(p, q) \otimes_{\mathbb{R}} \mathbb{K}$, whose classification is well known. For $\mathbb{K}=\mathbb{C}$, we have an isomorphism of algebras $\mathrm{Cl}_{\mathbb{C}}(p, q) \approx \mathrm{Cl}_{\mathbb{C}}(d, 0) \stackrel{\text { def }}{=} \mathrm{Cl}_{\mathbb{C}}(d)$ and the classification depends only on the $\bmod 2$ reduction of $d$; for $\mathbb{K}=\mathbb{R}$, it depends on the mod 8 reduction of $p-q$. The Schur algebra $\mathbb{S}_{\mathbb{K}}(p, q)$ is the largest division algebra contained in the center of $\mathrm{Cl}_{\mathbb{K}}(p, q)$; it is determined up to isomorphism of algebras, being isomorphic with $\mathbb{R}, \mathbb{C}$, or $\mathbb{H}$. The Clifford algebra is either simple (in which case it is isomorphic with a matrix algebra $\left.\operatorname{Mat}\left(\Delta_{\mathbb{K}}(d), \mathbb{S}_{\mathbb{K}}(p, q)\right)\right)$ or a direct sum of two central simple algebras (namely, the direct sum $\left.\operatorname{Mat}\left(\Delta_{\mathbb{K}}(d), \mathbb{S}_{\mathbb{K}}(p, q)\right) \oplus \operatorname{Mat}\left(\Delta_{\mathbb{K}}(d), \mathbb{S}_{\mathbb{K}}(p, q)\right)\right)$, where the positive integers $\Delta_{\mathbb{K}}(d)$ are given by well-known formulas recalled below. We say that the Clifford algebra is normal if its Schur algebra is isomorphic to the base field. It is convenient for our purpose to organize the various cases according to the isomorphism type of the Schur algebra and to whether the Clifford algebra is simple or not.

When $\mathbb{K}=\mathbb{C}$. In this case, the Schur algebra is always isomorphic with $\mathbb{C}\left(\right.$ so $\mathrm{Cl}_{\mathbb{C}}(p, q) \approx \mathrm{Cl}_{\mathbb{C}}(d)$ is always normal) and we always have $\nu \diamond v=+1$ and $\Delta_{\mathbb{C}}(d)=2^{[d / 2]}$. Moreover we have the following:

(i) The algebra is simple iff $d=$ even, in which case $\mathrm{Cl}_{\mathbb{C}}(d) \approx \operatorname{Mat}\left(\Delta_{\mathbb{C}}(d), \mathbb{C}\right)$ and $\nu$ is noncentral.

(ii) The algebra is nonsimple iff $d=$ odd, in which case $\mathrm{Cl}_{\mathbb{C}}(d) \approx \operatorname{Mat}\left(\Delta_{\mathbb{C}}(d), \mathbb{C}\right) \oplus \operatorname{Mat}\left(\Delta_{\mathbb{C}}(d), \mathbb{C}\right)$ and $v$ is central.

When $\mathbb{K}=\mathbb{R}$, we have the following:

(1) The Schur algebras and the numbers $\Delta_{\mathbb{R}}(d)$ are as follows (see, e.g., [28]):

(i) $\mathbb{S} \approx \mathbb{R}$ (normal case), which occurs iff $p-$ $q \equiv_{8} 0,1,2$ and we have $\Delta_{\mathbb{R}}(d)=2^{[d / 2]}$. 
TABLE 1: Properties of $v$ according to the mod 8 reduction of $p-q$ for the case $\mathbb{K}=\mathbb{R}$. At the intersection of each row and column, we indicate the values of $p-q(\bmod 8)$ for which the modified volume form $v$ has the corresponding properties. In parentheses, we also indicate the isomorphism type of the Schur algebra for that value of $p-q(\bmod 8)$. The real Clifford algebra $\operatorname{cl}(p, q)$ is nonsimple iff $p-q \equiv_{8} 1,5$, which corresponds to the upper left corner of the table. Notice that $v$ is central iff $d$ is odd.

\begin{tabular}{lll}
\hline $\mathbb{K}=\mathbb{R}$ & $\nu \diamond v=+1$ & $v \diamond v=-1$ \\
\hline$\nu$ is central & $1(\mathbb{R}), 5(\mathbb{M})$ & $3(\mathbb{C}), 7(\mathbb{C})$ \\
$\nu$ is not central & $0(\mathbb{R}), 4(\mathbb{M})$ & $2(\mathbb{R}), 6(\mathbb{M})$ \\
\hline
\end{tabular}

(ii) $\mathbb{S} \approx \mathbb{C}$ (almost complex case), which occurs iff $p-q \equiv_{8} 3,7$ and we have $\Delta_{\mathbb{R}}(d)=2^{[d / 2]}$.

(iii) $\mathbb{S} \approx \mathbb{4}$ (quaternionic case), which occurs iff $p-$ $q \equiv_{8} 4,5,6$ and we have $\Delta_{\mathbb{R}}(d)=2^{[d / 2]-1}$.

(2) The simple and nonsimple cases occur as follows:

(i) $\mathrm{Cl}(p, q)$ is simple iff $p-q \equiv_{8} 0,2,3,4,6,7$.

(ii) $\mathrm{Cl}(p, q)$ is nonsimple iff $p-q \equiv_{8} 1,5$. In this case, we always have $v \diamond \nu=+1$ and $\nu$ is central.

The situation when $\mathbb{K}=\mathbb{R}$ is summarized in Table 1. For both $\mathbb{K}=\mathbb{R}$ and $\mathbb{K}=\mathbb{C}$, the Clifford algebra is nonsimple iff $\nu$ is central and satisfies $v \diamond v=1$. In this case-for both $\mathbb{K}=$ $\mathbb{R}$ and $\mathbb{K}=\mathbb{C}$-the Clifford algebra admits two inequivalent irreducible representations by $\mathbb{K}$-linear operators, which are related by the main automorphism of the Clifford algebra and both of which are nonfaithful; their Schur algebra equals $\mathbb{C}$ when $\mathbb{K}=\mathbb{C}$ but may equal either $\mathbb{R}$ or $\mathbb{M}$ when $\mathbb{K}=\mathbb{R}$.

3.9. Twisted (Anti-)Self-Dual Forms in the Nonsimple Case. In this subsection, let us assume that we are in the nonsimple case. Then $v \diamond v=+1$ and (since $d$ is odd in the nonsimple case) $v$ is a central element of the Kähler-Atiyah algebra:

$$
\nu \diamond \omega=\omega \diamond \nu, \quad \forall \omega \in \Omega_{\mathbb{K}}(M) .
$$

Using the fact that $v$ is central, an easy computation shows that $P_{+}$and $P_{-}$are (nonunital) algebra endomorphisms of the Kähler-Atiyah algebra; in fact

$$
\begin{aligned}
P_{ \pm}(\omega \diamond \eta) & =P_{ \pm}(\omega) \diamond P_{ \pm}(\eta)=P_{ \pm}(\omega) \diamond \eta \\
& =\omega \diamond P_{ \pm}(\eta), \quad \forall \omega, \eta \in \Omega_{\mathbb{K}}(M), \\
P_{ \pm}\left(1_{M}\right) & =P_{ \pm} .
\end{aligned}
$$

In this case, $\Omega_{\mathbb{K}}^{ \pm}(M)$ are complementary two-sided ideals of the Kähler-Atiyah algebra (indeed, $p_{ \pm}$are central); in particular, $\left(\Omega_{\mathbb{K}}^{ \pm}(M), \diamond\right)$ are unital algebras, their units being given by $p_{ \pm}$.

Truncation and Prolongation. Since $d$ is odd in the nonsimple case, we have the decomposition:

$$
\Omega_{\mathbb{K}}(M)=\Omega_{\mathbb{K}}^{<}(M) \oplus \Omega_{\mathbb{K}}^{>}(M),
$$

where

$$
\begin{gathered}
\Omega_{\mathbb{K}}^{<}(M) \stackrel{\text { def }}{=} \oplus_{k=0}^{[d / 2]} \Omega_{\mathbb{K}}^{k}(M), \\
\Omega_{\mathbb{K}}^{>}(M) \stackrel{\text { def }}{=} \oplus_{k=[d / 2]+1}^{d} \Omega_{\mathbb{K}}^{k}(M) .
\end{gathered}
$$

The corresponding complementary $\mathscr{C}^{\infty}(M, \mathbb{R})$-linear idempotent operators $P_{<}, P_{>}: \Omega_{\mathbb{K}}(M) \rightarrow \Omega_{\mathbb{K}}(M)$ are given by

$$
\begin{aligned}
& P_{<}(\omega) \stackrel{\text { def }}{=} \omega^{<}, \\
& P_{>}(\omega) \stackrel{\text { def }}{=} \omega^{>},
\end{aligned}
$$

where, for any $\omega=\sum_{k=0}^{d} \omega^{(k)} \in \Omega_{\mathbb{K}}(M)\left(\right.$ with $\left.\omega^{(k)} \in \Omega_{\mathbb{K}}^{k}(M)\right)$, we define $\omega^{<}$(the lower truncation of $\omega$ ) and $\omega^{>}$(the upper truncation of $\omega$ ) through

$$
\begin{gathered}
\omega^{<} \stackrel{\text { def }}{=} \sum_{k=0}^{[d / 2]} \omega^{(k)}, \\
\omega^{>} \stackrel{\text { def }}{=} \sum_{k=[d / 2]+1}^{d} \omega^{(k)} .
\end{gathered}
$$

We have

$$
\begin{aligned}
& P_{>}+P_{<}=\operatorname{id}_{\Omega_{K}(M)}, \\
& P_{>} \circ P_{<}=P_{<} \circ P_{>}=0, \\
& P_{>} \circ P_{>}=P_{>}, \\
& P_{<} \circ P_{<}=P_{<} .
\end{aligned}
$$

When $\omega$ is twisted (anti-)self-dual (i.e., $\omega \in \Omega_{\nwarrow}^{\epsilon}(M)$ with $\epsilon=$ \pm 1 ), we have $\widetilde{*} \omega=\epsilon \omega$, which implies

$$
\omega^{>}=\epsilon \widetilde{*}\left(\omega^{<}\right), \quad \forall \omega \in \Omega_{\mathbb{K}}^{\epsilon}(M) .
$$

Hence in this case $\omega$ can be reconstructed from its lower truncation as

$$
\begin{aligned}
& \omega=\omega^{<}+\epsilon \widetilde{*}\left(\omega^{<}\right)=2 P_{\epsilon}\left(\omega^{<}\right)=P_{\epsilon}\left(2 P_{<}(\omega)\right), \\
& \forall \omega \in \Omega_{\mathbb{K}}^{\epsilon}(M) .
\end{aligned}
$$

It follows that the restriction of $2 P_{<}$to the subspace $\Omega_{\nwarrow}^{\epsilon}(M)$ gives a $\mathscr{C}^{\infty}(M, \mathbb{R})$-linear bijection between this subspace and the subspace $\Omega_{\mathbb{K}}^{<}(M)$, with inverse given by the restriction of $P_{\epsilon}$ to $\Omega_{\mathbb{K}}^{<}(M)$. We define the twisted (anti-)self-dual prolongation of a form $\omega \in \Omega_{\mathbb{K}}^{<}(M)$ through

$$
\omega_{ \pm} \stackrel{\text { def }}{=} P_{ \pm}(\omega), \quad \forall \omega \in \Omega_{\mathbb{K}}^{<}(M) .
$$

Of course, the form $\omega \in \Omega_{\mathbb{K}}^{<}(M)$ can be recovered from its two prolongations as $\omega=\omega_{+}+\omega_{-}$.

The Truncated Algebra $\left(\Omega_{\mathbb{1}}^{<}(M), \bullet_{ \pm}\right)$. We stress that $P_{<}$does not preserve the geometric product on its entire domain of definition $\Omega_{\mathbb{K}}(M)$; in fact, its image $\Omega_{\mathbb{K}}^{<}(M)$ is not 
a subalgebra of the Kähler-Atiyah algebra since it is not stable with respect to $\diamond$-multiplication. To cure this problem, we use the linear isomorphisms mentioned above to transfer the multiplication $\diamond$ of the unital subalgebra $\Omega_{\mathbb{K}}^{\epsilon}(M)$ to an associative and unital multiplication $\diamond_{\epsilon}$ defined on $\Omega_{\mathbb{K}}^{<}(M)$ through

$$
\begin{aligned}
\omega \diamond_{\epsilon} \eta & =2 P_{<}\left(P_{\epsilon}(\omega) \diamond P_{\epsilon}(\eta)\right) \in \Omega_{\mathbb{K}}^{<}(M) \Longleftrightarrow \\
P_{\epsilon}\left(\omega \diamond_{\epsilon} \eta\right) & =P_{\epsilon}(\omega) \diamond P_{\epsilon}(\eta),
\end{aligned}
$$

$$
\forall \omega, \eta \in \Omega_{\mathbb{K}}^{<}(M) .
$$

Since $P_{\epsilon}$ is a morphism of algebras on its entire domain of definition $\Omega_{\mathbb{K}}(M)$, we have $P_{\epsilon}(\omega) \diamond P_{\epsilon}(\eta)=P_{\epsilon}(\omega \diamond \eta)$, so (104) gives

$$
\omega \diamond_{\epsilon} \eta=2 P_{<}\left(P_{\epsilon}(\omega \diamond \eta)\right), \quad \forall \omega, \eta \in \Omega_{\mathbb{K}}^{<}(M) .
$$

Since $2 P_{\epsilon}(\omega \diamond \eta)=(1+\epsilon \widetilde{*})\left(P_{<}(\omega \diamond \eta)+P_{>}(\omega \diamond \eta)\right)$ and $\widetilde{*} \circ P_{<}=P_{>} \circ \widetilde{*}$, this implies

$$
\begin{aligned}
\omega \diamond \epsilon \eta & =P_{<}(\omega \diamond \eta)+\epsilon \widetilde{*} P_{>}(\omega \diamond \eta) \\
& =(\omega \diamond \eta)^{<}+\epsilon \widetilde{*}\left[(\omega \diamond \eta)^{>}\right],
\end{aligned}
$$

a formula which can be used to implement the product $\boldsymbol{}_{\epsilon}$ in a symbolic computation system. Combining everything shows that we have mutually inverse isomorphisms of algebras:

$$
\left(\Omega_{\mathbb{K}}^{<}(M), \diamond_{\epsilon}\right) \underset{2 P_{<} \Lambda_{\Omega_{\mathbb{K}}^{\epsilon}(M)}}{\stackrel{\left.P_{\epsilon}\right|_{\Omega_{\mathbb{K}}^{<}(M)}}{\rightleftarrows}}\left(\Omega_{\mathbb{K}}^{\epsilon}(M), \diamond\right) .
$$

Thus $\left(\Omega_{\mathbb{K}}^{<}(M), \diamond_{\epsilon}\right)$ provides a model for the unital associative algebra $\left(\Omega_{\mathbb{K}}^{\epsilon}(M), \diamond\right)$.

Local Expansions. Let us further assume that $d \geq 3$. Then the covector fields $e^{a} \in \Omega_{\mathbb{K}}^{1}(M)$ defined by a local pseudo-orthonormal frame above $U \subset M$ belong to the subspace $\Omega_{\mathbb{K}}^{<}(M)$ and we consider their twisted (anti-)selfdual prolongations:

$$
e_{ \pm}^{a} \stackrel{\text { def }}{=} P_{ \pm}\left(e^{a}\right) \in \Omega_{\mathbb{K}}^{ \pm}(M) .
$$

Since $e^{a_{1} \cdots a_{k}}=e^{a_{1}} \wedge \cdots \wedge e^{a_{k}}=e^{a_{1}} \diamond \cdots \diamond e^{a_{k}}$ and since $P_{ \pm}$ are endomorphisms of the Kähler-Atiyah algebra, we find that the prolongations of $e^{a_{1} \cdots a_{k}}$ are given by $\diamond$-monomials in the prolongations of $e^{a}$ :

$$
e_{ \pm}^{a_{1} \cdots a_{k}} \stackrel{\text { def }}{=} P_{ \pm}\left(e^{a_{1} \cdots a_{k}}\right)=e_{ \pm}^{a_{1}} \diamond \cdots \diamond e_{ \pm}^{a_{k}} .
$$

In particular, the twisted (anti-)self-dual forms $\left\{e_{ \pm}^{a_{1} \cdots a_{k}} \mid 1 \leq\right.$ $\left.a_{1}<\cdots<a_{k} \leq d, k=0, \ldots,[d / 2]\right\}$ constitute a basis of the free $\mathscr{C}^{\infty}(U, \mathbb{K})$-module $\Omega_{\mathbb{K}}^{ \pm}(U)$ (since $\left\{e^{a_{1} \cdots a_{k}} \mid 1 \leq a_{1}<\right.$ $\left.\ldots<a_{k} \leq d, k=0, \ldots,[d / 2]\right\}$ form a basis of the module $\Omega_{\mathbb{K}}^{<}(U)$ and since the operation of taking the prolongation is an isomorphism of $\mathscr{C}^{\infty}(U, \mathbb{K})$-modules). In fact, any twisted (anti-)self-dual form $\omega \in \Omega_{\mathbb{K}}^{ \pm}(M)$ (cf. (2), (3)) expands as

$$
\omega={ }_{U} 2 \sum_{k=0}^{[d / 2]} \frac{1}{k !} \omega_{a_{1} \cdots a_{k}}^{(k)} e_{ \pm}^{a_{1} \cdots a_{k}}, \quad \forall \omega \in \Omega_{\mathbb{K}}^{ \pm}(M),
$$

where $\omega_{a_{1} \cdots a_{k}}^{(k)} \in \mathscr{C}^{\infty}(U, \mathbb{K})$ are as in (3). The coefficients with $k \geq[d / 2]+1$ are determined by those with $k \leq[d / 2]$ through relations (92). The lower truncation of such $\omega$ has the local expansion:

$$
\omega^{<}=\sum_{k=0}^{[d / 2]} \frac{1}{k !} \omega_{a_{1} \cdots a_{k}}^{(k)} e^{a_{1} \cdots a_{k}}
$$

We also note the explicit expressions:

$$
e_{ \pm}^{a_{1} \cdots a_{k}}=\frac{1}{2}\left(e^{a_{1} \cdots a_{k}} \pm c_{p, q}(\mathbb{K}) \epsilon^{a_{k} \cdots a_{1}} a_{k+1} \cdots a_{d} e^{a_{k+1} \cdots a_{d}}\right)
$$

where $c_{p, \mathrm{q}}(\mathbb{K})$ was defined in (78).

3.10. Orthogonality in the Nonsimple Case. Assuming that we are in the nonsimple case, let us consider the situation when we have a distinguished one-form $\theta \in \Omega_{\mathbb{K}}^{1}(M)$ which satisfies the normalization condition $\iota_{\theta} \theta=1$.

The Isomorphism of Algebras between $\Omega_{\mathbb{K}}^{\perp}(M)$ and $\Omega_{\mathbb{K}}^{\epsilon}(M)$. For any $\omega \in \Omega_{\mathbb{K}}(M)$, consider the decomposition $\omega=\omega_{\|}+\omega_{\perp}$ into parts $\omega_{\|}=\theta \wedge\left(\iota_{\theta} \omega\right)$ and $\omega_{\perp}=\iota_{\theta}(\theta \wedge \omega)=\omega-\omega_{\|}$parallel and perpendicular to $\theta$. Since $\theta \| \nu$ (indeed, we have $\theta \wedge \nu=0$ ) and $\widetilde{*} \omega=\omega \diamond \nu$, properties (61) imply

$$
\begin{aligned}
& \theta \| \widetilde{*}\left(\omega_{\perp}\right), \\
& \theta \perp \widetilde{*}\left(\omega_{\|}\right),
\end{aligned}
$$

which gives

$$
\begin{aligned}
& (\widetilde{*} \omega)_{\|}=\widetilde{*}\left(\omega_{\perp}\right), \\
& (\widetilde{*} \omega)_{\perp}=\widetilde{*}\left(\omega_{\|}\right) .
\end{aligned}
$$

The subalgebras $\Omega_{\mathbb{K}}^{\epsilon}(M)$ and $\Omega_{\mathbb{K}}^{\perp}(M)$ of the Kähler-Atiyah algebra can be identified with each other using the operator $2 P_{\perp}$, which takes a twisted (anti-)self-dual form $\omega$ into $2 \omega_{\perp}$. Indeed, if $\widetilde{*} \omega=\epsilon \omega$ (with $\epsilon= \pm 1$ ), then $\widetilde{*}\left(\omega_{\|}\right)=\epsilon \omega_{\perp}$ and $\widetilde{*}\left(\omega_{\perp}\right)=\epsilon \omega_{\|}$. Hence the last of (62) implies

$$
(\omega \diamond \eta)_{\perp}=\widetilde{*}\left(\omega_{\perp}\right) \diamond \widetilde{*}\left(\eta_{\perp}\right)+\omega_{\perp} \diamond \eta_{\perp}=2 \omega_{\perp} \diamond \eta_{\perp}
$$

since $\widetilde{*}\left(\omega_{\perp}\right) \diamond \widetilde{*}\left(\eta_{\perp}\right)=\omega_{\perp} \diamond \nu \diamond \eta_{\perp} \diamond v=\omega_{\perp} \diamond \eta_{\perp} \diamond v \diamond v=$ $\omega_{\perp} \diamond \eta_{\perp}$, where we used the fact that $\nu$ is central in the KählerAtiyah algebra and that it squares to $1_{M}$. Thus

$$
\begin{aligned}
(\omega \diamond \eta)_{\perp} & =2 \omega_{\perp} \diamond \eta_{\perp} \Longleftrightarrow \\
2 P_{\perp}(\omega \diamond \eta) & =2\left(2 P_{\perp} \omega\right) \diamond\left(2 P_{\perp} \eta\right),
\end{aligned}
$$

$$
\forall \omega, \eta \in \Omega_{\mathbb{K}}^{\epsilon}(M) .
$$

We also have

$$
2 P_{\perp}\left(p_{ \pm}\right)=P_{\perp}(1 \pm v)=P_{\perp}\left(1_{M}\right)=1_{M},
$$

where we used the fact that $\theta \| v$. These properties show that the restriction $\left.2 P_{\perp}\right|_{\Omega_{\mathrm{K}}^{e}(M)}$ is a unital morphism of algebras from $\left(\Omega_{\mathbb{K}}^{\epsilon}(M), \diamond\right)$ to $\left(\Omega_{\mathbb{K}}^{\perp}(M), \diamond\right)$. An easy computation shows 
that it is an isomorphism whose inverse equals the restriction of $P_{\epsilon}$ to $\Omega_{\mathbb{K}}^{\perp}(M)$. It follows that we have mutually inverse unital isomorphisms of algebras:

$$
\left(\Omega_{\mathbb{K}}^{\epsilon}(M), \diamond\right) \underset{\left.P_{\epsilon}\right|_{\Omega_{\mathbb{K}}^{\perp}(M)}}{\stackrel{\left.2 P_{\perp}\right|_{\Omega_{\mathbb{K}}^{\epsilon}(M)}}{\rightleftarrows}}\left(\Omega_{\mathbb{K}}^{\perp}(M), \diamond\right) .
$$

Combining with the results of Section 3.9, we have thus found two isomorphic models for the unital subalgebra $\left(\Omega_{\mathbb{K}}^{\epsilon}(M), \diamond\right)$ :

$$
\left(\Omega_{\mathbb{K}}^{<}(M), \diamond_{\epsilon}\right) \underset{2 P_{<} \operatorname{l}_{\mathbb{K}}^{\epsilon}(M)}{\stackrel{\left.P_{\epsilon}\right|_{\Omega_{K}^{<}(M)}}{\rightleftarrows}}\left(\Omega_{\mathbb{K}}^{\epsilon}(M), \diamond\right) \underset{\left.P_{\epsilon}\right|_{\Omega_{\mathbb{K}}^{\perp}(M)}}{\stackrel{\left.2 P_{\perp}\right|_{\Omega_{K}^{\epsilon}} ^{\epsilon}(M)}{\rightleftarrows}}\left(\Omega_{\mathbb{K}}^{\perp}(M), \diamond\right) .
$$

The Reduced Twisted Hodge Operator. Since $\theta \| v$, we can write

$$
v=\theta \wedge v_{\mathrm{T}}
$$

where the reduced volume form $\nu_{\mathrm{T}}$ is defined through

$$
\nu_{\top} \stackrel{\text { def }}{=} \iota_{\theta} v=\theta \diamond \nu=v \diamond \theta \text {. }
$$

The last two equalities in (121) follow from (29) and from the fact that (in the nonsimple case) $v$ is central in the KählerAtiyah algebra (since $d$ is odd in this case). Multiplying the last equation with $\theta$ in the Kähler-Atiyah algebra and using the fact that $\theta \diamond \theta=\widehat{g}(\theta, \theta)=1$ give

$$
\nu=\nu_{\mathrm{T}} \diamond \theta=\theta \diamond \nu_{\mathrm{T}} .
$$

Notice the identity:

$$
v_{\top}^{2}=+1_{M},
$$

which follows from (121) using the fact that $v$ is central, the normalization condition for $\theta$ and the property $\nu \diamond \nu=$ $+1_{M}$, which always holds in the nonsimple case. Defining the reduced twisted Hodge operator $\widetilde{*}_{0}$ through

$$
\begin{aligned}
& \widetilde{*}_{0} \omega \stackrel{\text { def }}{=} \pi(\omega) \diamond \nu_{\top}, \\
& \forall \omega \in \Omega_{\mathbb{K}}(M) \Longleftrightarrow \widetilde{*}_{0}=R_{\nu_{\top}} \circ \pi,
\end{aligned}
$$

we have $\pi\left(\nu_{\mathrm{T}}\right)=\nu_{\mathrm{T}}$, so (123) implies

$$
\widetilde{*}_{0} \circ \widetilde{*}_{0}=+\mathrm{id}_{\Omega_{\nwarrow}(M)} .
$$

For later reference, we note the identities (where we use (122) and the fact that $\left.\pi\left(\nu_{\mathrm{T}}\right)=\nu_{\mathrm{T}}\right)$

$$
\left[\pi, R_{\nu_{\top}}\right]_{-, \circ}=\left[\pi, \widetilde{*}_{0}\right]_{-, \circ}=0
$$

as well as

$$
\left[L_{\theta}, \widetilde{*}_{0}\right]_{+, \circ}=\left[R_{\theta}, \widetilde{*}_{0}\right]_{+, \circ}=0,
$$

which follow by easy computation. Using (48), the last identities imply the following anticommutation relations, which will be important below:

$$
\left[\wedge_{\theta}, \widetilde{*}_{0}\right]_{+, \circ}=\left[\iota_{\theta}, \widetilde{*}_{0}\right]_{+, \circ}=0 .
$$

To find explicit expressions for the parallel and perpendicular parts of $\widetilde{*} \omega$, notice that $\widetilde{*} \omega=\omega \diamond \nu=\omega \diamond \nu_{\top} \diamond \theta=\theta \wedge \pi(\omega \diamond$ $\left.\nu_{T}\right)-\iota_{\theta} \pi\left(\omega \diamond \nu_{T}\right)=\theta \wedge\left(\pi(\omega) \diamond \nu_{T}\right)-\iota_{\theta}\left(\pi(\omega) \diamond \nu_{T}\right)$, where we used (29) and the fact that $\pi\left(\nu_{T}\right)=+\nu_{T}$. Thus

$$
\begin{aligned}
& (\widetilde{*} \omega)_{\|}=\theta \wedge\left[\pi(\omega) \diamond \nu_{\mathrm{T}}\right]=\theta \wedge\left[\left(\pi(\omega) \diamond \nu_{\mathrm{T}}\right)_{\perp}\right], \\
& (\widetilde{*} \omega)_{\perp}=-\iota_{\theta}\left[\pi(\omega) \diamond \nu_{\top}\right]=-\iota_{\theta}\left[\left(\pi(\omega) \diamond \nu_{T}\right)_{\|}\right] .
\end{aligned}
$$

The decomposition $\omega=\omega_{\|}+\omega_{\perp}$ and the fact that $\iota_{\theta} \nu_{\top}=0$ (thus $\theta \perp \nu_{\top}$ ) imply (using (61))

$$
\begin{aligned}
& \theta \|\left(\omega_{\|} \diamond \nu_{\mathrm{T}}\right), \\
& \theta \perp\left(\omega_{\perp} \diamond \nu_{\mathrm{T}}\right),
\end{aligned}
$$

and (using (64) and the fact that $\pi\left(\nu_{\mathrm{T}}\right)=+1$ )

$$
\begin{aligned}
& \theta \|\left(\pi\left(\omega_{\|}\right) \diamond \nu_{\mathrm{T}}\right), \\
& \theta \perp\left(\pi\left(\omega_{\perp}\right) \diamond \nu_{\mathrm{T}}\right) .
\end{aligned}
$$

These relations show that

$$
\begin{aligned}
& \left(\omega \diamond v_{\top}\right)_{\|}=\omega_{\|} \diamond v_{\top}, \\
& \left(\omega \diamond v_{T}\right)_{\perp}=\omega_{\perp} \diamond v_{T}
\end{aligned}
$$

as well as

$$
\begin{aligned}
& \left(\pi(\omega) \diamond \nu_{T}\right)_{\|}=\pi\left(\omega_{\|}\right) \diamond \nu_{T}, \\
& \left(\pi(\omega) \diamond \nu_{T}\right)_{\perp}=\pi\left(\omega_{\perp}\right) \diamond \nu_{T} .
\end{aligned}
$$

Combining the last relation with (129) gives

$$
\begin{aligned}
& (\widetilde{* \omega})_{\|}=\theta \wedge\left[\pi\left(\omega_{\perp}\right) \diamond \nu_{\top}\right], \\
& (\widetilde{*} \omega)_{\perp}=-\iota_{\theta}\left[\pi\left(\omega_{\|}\right) \diamond \nu_{\top}\right] .
\end{aligned}
$$

Equations (134) and (114) read

$$
\begin{aligned}
& (\widetilde{*} \omega)_{\|}=\widetilde{*}\left(\omega_{\perp}\right)=\theta \wedge \widetilde{*}_{0}\left(\omega_{\perp}\right), \\
& (\widetilde{*} \omega)_{\perp}=\widetilde{*}\left(\omega_{\|}\right)=-\iota_{\theta} \widetilde{*}_{0}\left(\omega_{\|}\right),
\end{aligned}
$$

while (133) gives

$$
\begin{aligned}
& \left(\widetilde{*}_{0} \omega\right)_{\|}=\widetilde{*}_{0}\left(\omega_{\|}\right), \\
& \left(\widetilde{*}_{0} \omega\right)_{\perp}=\widetilde{*}_{0}\left(\omega_{\perp}\right) .
\end{aligned}
$$

In particular, we have

$$
\left[\widetilde{*}_{0}, P_{\|}\right]_{-, \circ}=\left[\widetilde{*}_{0}, P_{\perp}\right]_{-, \circ}=0
$$

and $\tilde{*}=\wedge_{\theta} \circ \widetilde{*}_{0} \circ P_{\perp}-\iota_{\theta} \circ \widetilde{*}_{0} \circ P_{\|}=\wedge_{\theta} \circ P_{\perp} \circ \widetilde{*}_{0}-\iota_{\theta} \circ P_{\|} \circ \widetilde{*}_{0}$, which gives

$$
\widetilde{*}=\Lambda_{\theta} \circ \widetilde{*}_{0}-\iota_{\theta} \circ \widetilde{*}_{0}
$$

upon using $\wedge_{\theta} \circ P_{\|}=\iota_{\theta} \circ P_{\perp}=0 \Leftrightarrow \wedge_{\theta} \circ P_{\perp}=\wedge_{\theta}$ and $\iota_{\theta} \circ P_{\|}=\iota_{\theta}$. The relations above imply the following. 
Lemma 6. Consider the operators $\alpha_{\theta} \stackrel{\text { def }}{=} \wedge_{\theta} \circ \widetilde{*}_{0}$ and $\beta_{\theta}=$ $-\iota_{\theta} \circ \widetilde{*}_{0}$. Then

$$
\begin{aligned}
& \alpha_{\theta} \circ \alpha_{\theta}=\beta_{\theta} \circ \beta_{\theta}=0, \\
& \alpha_{\theta} \circ \beta_{\theta}=P_{\|}, \\
& \beta_{\theta} \circ \alpha_{\theta}=P_{\perp} .
\end{aligned}
$$

Proof. The statement follows by direct computation using properties (128) and (125).

Notice that (135) takes the form

$$
\begin{aligned}
& (\tilde{*} \omega)_{\|}=\widetilde{*}\left(\omega_{\perp}\right)=\alpha_{\theta}\left(\omega_{\perp}\right), \\
& (\widetilde{*} \omega)_{\perp}=\widetilde{*}\left(\omega_{\|}\right)=\beta_{\theta}\left(\omega_{\|}\right) .
\end{aligned}
$$

For reader's convenience, we also list a few other properties which follow immediately from the above:

$$
\begin{aligned}
& \alpha_{\theta} \circ P_{\|}=0 \Longrightarrow \alpha_{\theta} \circ P_{\perp}=\alpha_{\theta}, \\
& P_{\perp} \circ \alpha_{\theta}=0 \Longrightarrow P_{\|} \circ \alpha_{\theta}=\alpha_{\theta}, \\
& \beta_{\theta} \circ P_{\perp}=0 \Longrightarrow \beta_{\theta} \circ P_{\|}=\beta_{\theta}, \\
& P_{\|} \circ \beta_{\theta}=0 \Longrightarrow P_{\perp} \circ \beta_{\theta}=\beta_{\theta}, \\
& P_{\|} \circ \widetilde{*}=\alpha_{\theta}, \\
& P_{\perp} \circ \tilde{*}=\beta_{\theta} .
\end{aligned}
$$

Proposition 7. Let $\omega \in \Omega_{\mathbb{K}}(M)$. Then the following statements are equivalent.

(a) $\omega$ is twisted (anti-)self-dual; that is, $\widetilde{*} \omega=\epsilon \omega$ for $\epsilon= \pm 1$.

(b) The components $\omega_{\|}$and $\omega_{\perp}$ satisfy the following equivalent relations:

$$
\begin{aligned}
& \omega_{\|}=\epsilon \theta \wedge \widetilde{*}_{0}\left(\omega_{\perp}\right), \\
& \omega_{\perp}=-\epsilon \iota_{\theta} \widetilde{*}_{0}\left(\omega_{\|}\right) .
\end{aligned}
$$

In this case, $\omega_{\|}$and $\omega_{\perp}$ determine each other and thus any of them determines $\omega$.

Proof. The fact that the two relations listed in (142) are equivalent to each other is an immediate consequence of the lemma. The rest of the proposition follows from (138).

Recalling definition (66), we have $\theta \perp \omega_{\top}$ and $\omega_{\|}=\wedge_{\theta} \omega_{\top}$, so the decomposition of $\omega$ reads $\omega=\theta \wedge \omega_{\top}+\omega_{\perp}$. Using (128), we find

$$
\beta_{\theta} \circ \wedge_{\theta}=P_{\perp} \circ \widetilde{*}_{0}=\widetilde{*}_{0} \circ P_{\perp}
$$

which implies

$$
\beta_{\theta}\left(\omega_{\|}\right)=\widetilde{*}_{0}\left(\omega_{\mathrm{T}}\right) \Longrightarrow(\widetilde{*} \omega)_{\perp}=\widetilde{*}\left(\omega_{\|}\right)=\widetilde{*}_{0}\left(\omega_{\mathrm{T}}\right) \text {, }
$$

where in the last equality we used (140). Hence the previous proposition has the following.
Corollary 8. The following statements are equivalent for any $\omega \in \Omega_{\mathbb{K}}(M)$.

(a) $\omega$ is twisted (anti-)self-dual; that is, $\widetilde{*} \omega=\epsilon \omega$ for $\epsilon= \pm 1$.

(b) The inhomogeneous forms $\omega_{\top}=\iota_{\theta} \omega$ and $\omega_{\perp}=\iota_{\theta}(\theta \wedge \omega)$ satisfy the equation

$$
\omega_{\perp}=\epsilon \widetilde{*}_{0} \omega_{\mathrm{T}} \Longleftrightarrow \omega_{\mathrm{T}}=\epsilon \widetilde{*}_{0} \omega_{\perp} .
$$

In this case, $\omega_{\perp}$ and $\omega_{\top}$ determine each other and thus any of them determines $\omega$. Explicitly, $\omega_{\top}$ determines $\omega$ through the formula

$$
\omega=\left(\wedge_{\theta}+\epsilon \widetilde{*}_{0}\right)\left(\omega_{\top}\right),
$$

while $\omega_{\perp}$ determines $\omega$ through

$$
\omega=\left(\operatorname{id}_{\Omega_{\mathbb{K}}(M)}+\epsilon \wedge_{\theta} \circ \widetilde{*}_{0}\right)\left(\omega_{\perp}\right) .
$$

The corollary shows that the maps $\wedge_{\theta}+\epsilon \widetilde{*}_{0}: \Omega_{\mathbb{K}}^{\perp}(M) \stackrel{\sim}{\rightarrow}$ $\Omega_{\mathbb{K}}^{\epsilon}(M)$ are isomorphisms of $\mathscr{C}^{\infty}(M, \mathbb{K})$-modules, whose inverses are given by $\omega \rightarrow \omega_{\mathrm{T}}$. We stress that these maps are not isomorphisms of algebras.

The Morphism $\varphi_{\epsilon}$. For later reference, consider the $\mathscr{C}^{\infty}(M, \mathbb{R})$-linear operator:

$$
\varphi_{\epsilon} \stackrel{\text { def }}{=} 2 P_{\perp} \circ P_{\epsilon}: \Omega_{\mathbb{K}}(M) \longrightarrow \Omega_{\mathbb{K}}^{\perp}(M)
$$

which acts as follows on $\omega=\theta \diamond \omega_{T}+\omega_{\perp}=\theta \wedge \omega_{T}+\omega_{\perp} \epsilon$ $\Omega_{\mathbb{K}}(M)$ :

$$
\varphi_{\epsilon}(\omega)=\epsilon \widetilde{*}_{0}\left(\omega_{\mathrm{T}}\right)+\omega_{\perp}=\epsilon \nu_{\mathrm{T}} \diamond \omega_{\mathrm{T}}+\omega_{\perp},
$$

where we used (144) and we noticed that $\nu_{T} \diamond \omega_{T}=\pi\left(\omega_{\top}\right) \diamond$ $\nu_{T}=\widetilde{*}_{0}\left(\omega_{\mathrm{T}}\right)$ (since $\omega_{\mathrm{T}}$ and $\nu_{\mathrm{T}}$ are orthogonal to $\theta$ and since $\operatorname{rk} \nu_{T}=d-1$ is even). We have $\varphi_{\epsilon}(\theta)=\epsilon \nu_{\top}$ ( since $\theta_{T}=1$ and $\left.\theta_{\perp}=0\right)$ and $\varphi_{\epsilon}(\omega)=\omega$ for all $\omega \in \Omega_{\mathbb{K}}^{\perp}(M)$; in fact, these properties determine $\varphi_{\epsilon}$. One has the following.

Proposition 9. The map $\varphi_{\epsilon}$ is idempotent; that is, $\varphi_{\epsilon} \circ \varphi_{\epsilon}=$ $\varphi_{\epsilon}$. Moreover, it is a (unital) morphism of algebras from $\left(\Omega_{\mathbb{K}}(M), \diamond\right)$ to $\left(\Omega^{\perp}(M), \diamond\right)$.

Proof. Idempotency follows by noticing that $\left.\varphi_{\epsilon}\right|_{\Omega_{\nwarrow}^{\perp}(M)}=$ $\operatorname{id}_{\Omega_{\mathbb{K}}^{\perp}(M)}$. The fact that $\varphi_{\epsilon}$ is a morphism of algebras follows since both $P_{\perp}$ and $P_{\epsilon}$ are such. Finally, unitality of $\varphi_{\epsilon}$ follows by computing:

$$
\varphi_{\epsilon}\left(1_{M}\right)=P_{\perp}\left(1_{M}+\epsilon \nu\right)=1_{M},
$$

where we used $P_{\perp}\left(1_{M}\right)=1_{M}$ and $P_{\perp}(\nu)=0$.

It is clear that $\varphi_{\epsilon}$ is surjective. Moreover, the last proposition of the previous paragraph implies

$$
\mathscr{K}\left(\varphi_{\epsilon}\right)=\Omega_{\mathbb{K}}^{-\epsilon}(M)
$$


It follows that $\varphi_{\epsilon}$ restricts to an isomorphism from $\Omega_{\mathbb{K}}^{\epsilon}(M)$ to $\Omega_{\mathbb{K}}^{\perp}(M)$-which, of course, equals the isomorphism $\left.2 P_{\perp}\right|_{\Omega_{\aleph}^{e}(M)}$ of diagram (118). Notice the relations:

$$
\begin{gathered}
P_{\epsilon} \circ \varphi_{\epsilon}=P_{\epsilon}, \\
\varphi_{\epsilon} \circ P_{\epsilon}=\varphi_{\epsilon}, \\
P_{\perp} \circ \varphi_{\epsilon}=\varphi_{\epsilon}, \\
\varphi_{\epsilon} \circ P_{\perp}=P_{\perp},
\end{gathered}
$$

where the first equality follows from the fact that $2 P_{\epsilon} \circ P_{\perp}$ restricts to the identity on $\Omega_{\mathbb{K}}^{\epsilon}(M)$ (see diagram (118)). Also notice the property:

$$
\varphi_{\epsilon}(\nu)=\epsilon 1_{M},
$$

which follows by direct computation upon using $P_{\perp}\left(1_{M}\right)=$ $1_{M}, P_{\perp}(\nu)=0$ and the fact that $\nu \diamond \nu=1_{M}$.

\section{Describing Bundles of Pinors}

In this section, we discuss the realization of pin bundles within the geometric algebra formalism-focusing especially on the nonsimple case, when the irreducible pin representations are nonfaithful. Section 4.1 discusses an approach to pinor bundles which is particularly well adapted to the geometric algebra formalism. In this approach (which, in some ways, goes back to Dirac; see $[18,29]$ for a beautiful treatment), one defines pinors as sections of a bundle $S$ of modules over the Kähler-Atiyah algebra, the fiberwise module structure being described by a morphism $\gamma$ : $\left(\Omega_{\mathbb{K}}(M), \diamond\right) \rightarrow(\operatorname{End}(S), \circ)$ of bundles of algebras. For the case when $\gamma$ is irreducible on the fibers, the well-known representation theory relevant for this construction and its relation with the fiber type classification of the KählerAtiyah bundle is recalled in Section 4.2, paying attention to characterizing the kernel and image of $\gamma$. In Section 4.3, we introduce a certain "partial inverse" $\gamma^{-1}$ of $\gamma$, which provides a sort of "vertical dequantization" map. Section 4.4 discusses a trace on the subalgebra $\Omega_{\mathbb{K}}^{\gamma}(M)$, which is related by $\gamma$ to the natural fiberwise trace on the pin bundle.

4.1. Basic Considerations. We define a bundle of $\mathbb{K}$-pinors to be a bundle $S$ of modules over the Clifford bundle $\mathrm{Cl}\left(T_{\mathbb{K}}^{*} M\right)$, that is, a $\mathbb{K}$-vector bundle all of whose fibers $S_{x}(x \in M)$ are modules over the corresponding Clifford algebras $\mathrm{Cl}\left(T_{\mathbb{K}, x}^{*} M\right)$. In our language, such a bundle is simply a bundle of modules over the Kähler-Atiyah bundle $\left(\wedge T_{\mathbb{K}}^{*} M, \diamond\right)$. In the particular case when the morphism $\gamma:\left(\wedge T_{\mathbb{K}}^{*} M, \diamond\right) \rightarrow \operatorname{End}(S)$ induces an irreducible representation of the Clifford algebra $\mathrm{Cl}\left(T_{\mathbb{K}, x}^{*} M\right)$ on each fiber $\operatorname{End}\left(S_{x}\right)$, a bundle of pinors will be called a pin bundle. Bundles of $\mathbb{K}$-spinors and $\mathbb{K}$-spin bundles are defined similarly, but replacing $\mathrm{Cl}\left(T_{\mathbb{K}}^{*} M\right)$ with $\mathrm{Cl}^{\mathrm{ev}}\left(T_{\mathbb{K}}^{*} M\right)$, that is, replacing the Kähler-Atiyah bundle with its even subbundle. Physically, smooth sections of a (s)pin bundle describe $\mathbb{K}$-valued (s)pinors of spin $1 / 2$ defined over the manifold $M$. As explained in $[18,29]$, a pin bundle $S$ in our sense exists on $M$ iff $M$ admits a so-called Clifford ${ }^{c}$ structure, that is, a "reduction" of the structure group of the bundle of pseudo-orthonormal frames of $T_{\mathbb{K}} M$ to a certain extension of the Clifford (a.k.a. Lipschitz) group over $\mathbb{K}$. In this case, the choices globally available for $S$ depend-up to isomorphism-on the choices of a Clifford ${ }^{c}$ structure and can be obtained from such a structure by applying the associated bundle construction. The map induced on sections satisfies

$$
\gamma(\omega \diamond \eta)=\gamma(\omega) \circ \gamma(\eta), \quad \forall \omega, \eta \in \Omega_{\mathbb{K}}(M)
$$

as well as

$$
\gamma\left(1_{M}\right)=\mathrm{id}_{S}
$$

where $\operatorname{id}_{S} \in \Gamma(M, \operatorname{End}(S))$ denotes the identity section of the bundle $\operatorname{End}(S)$.

In the language of "vertical quantization" of spin systems, the $\left(L^{2}\right.$-completion of the) space $\Gamma(M, S)$ of smooth sections of $S$ plays the role of the Hilbert space (when $\mathbb{K}=\mathbb{R}$, one of course has to consider the complexification of $S$ instead). In this interpretation, $\gamma$ plays the role of quantization map, giving a morphism from the algebra of quantum observables of the system (which is the Kähler-Atiyah algebra) to the $\left(L^{2}\right.$ completion of the) algebra $(\Gamma(M, \operatorname{End}(S)), \circ)$, which plays the role of algebra of "vertical" operators acting in the Hilbert space. These statements can be made quite precise provided that certain global conditions are imposed on $(M, g)$, but the rigorous treatment of this issue falls outside of the scope of this paper.

Notation 1. If $\left(e_{a}\right)_{a=1 \cdots d}$ is a local frame of TM above an open subset $U \subset M$ (with dual coframe $\left(e^{a}\right)_{a=1 \ldots d}$ ), then any inhomogeneous differential form $\omega$ on $M$ expands locally as in (2). We define $\gamma^{a} \stackrel{\text { def }}{=} \gamma\left(e^{a}\right) \in \Gamma(U$, End $(S))$, so $\gamma\left(e^{a_{1} \cdots a_{k}}\right)=$ $\gamma^{a_{1} \cdots a_{k}} \stackrel{\text { def }}{=}(1 / k !) \epsilon_{a_{1} \cdots a_{k}} \gamma^{a_{1}} \circ \cdots \circ \gamma^{a_{k}} \in \Gamma(U, \operatorname{End}(S))$ (the complete antisymmetrization of the composition $\left.\gamma^{a_{1}} \circ \ldots \circ \gamma^{a_{k}}\right)$. We have

$$
\gamma(\omega) \stackrel{\text { def }}{=}_{U} \sum_{k=0}^{d} \frac{1}{k !} \omega_{a_{1} \cdots a_{k}}^{(k)} \gamma^{a_{1} \cdots a_{k}}
$$

The locally defined sections $\gamma^{a} \in \Gamma(U$, End $(S))$ correspond to physicists' "gamma matrices."

4.2. Representation Theory. Let $S$ be a pin bundle with underlying morphism $\gamma:\left(\Omega_{\mathbb{K}}(M), \diamond\right) \rightarrow(\operatorname{End}(S), \circ)$.

Injectivity and Surjectivity of $\gamma$. It is important to note that $\gamma$ need not be fiberwise injective or surjective; that is, the morphisms of algebras $\gamma_{x}:\left(\Lambda T_{\mathbb{K}, x}^{*} M, \diamond_{x}\right) \approx \mathrm{Cl}\left(T_{\mathbb{K}, x}^{*} M\right) \rightarrow$ $\operatorname{End}\left(S_{x}\right)$ need not be injective or surjective. The following characterization is convenient in this regard:

(i) $\gamma$ is fiberwise injective iff the fiber of the KählerAtiyah bundle is simple as an associative algebra.

(ii) $\gamma$ is fiberwise surjective iff the Schur algebra of the fiber of the Kähler-Atiyah bundle is isomorphic with the base field $\mathbb{K}$. 
TABLE 2: Fiberwise character of $\gamma$ for the case $\mathbb{K}=\mathbb{R}$. At the intersection of each row and column, we indicate the values of $p-q$ (mod 8) for which the map induced by $\gamma$ on each fiber of the KählerAtiyah algebra has the corresponding properties. In parentheses, we also indicate the isomorphism type of the Schur algebra for that value of $p-q(\bmod 8)$. Note that $\gamma$ is fiberwise surjective exactly for the normal case, that is, when the Schur algebra is isomorphic with $\mathbb{R}$.

\begin{tabular}{lcc}
\hline $\mathbb{K}=\mathbb{R}$ & Injective & Noninjective \\
\hline Surjective & $0(\mathbb{R}), 2(\mathbb{R})$ & $1(\mathbb{R})$ \\
Nonsurjective & $3(\mathbb{C}), 7(\mathbb{C}), 4(\mathbb{M}), 6(\mathbb{M})$ & $5(\mathbb{M})$ \\
\hline
\end{tabular}

This gives the following classification.

The Case $\mathbb{K}=\mathbb{C}$. Then $\gamma$ is always fiberwise surjective, being fiberwise injective iff $d$ is even.

The Case $\mathbb{K}=\mathbb{R}$. Then $\gamma$ is fiberwise surjective iff $p-$ $q \equiv_{8} 0,1,2$. It is fiberwise injective iff $p-q \equiv_{8} 0,2,3,4,6,7$. This is summarized in Table 2.

The Schur Bundle and the Image of $\gamma$. The Schur algebra $\mathbb{S}_{\mathbb{K}}(p, q)$ of Section 3.8 is realized naturally in the representation space. Picking a point $x$ on $M$, let $\Sigma_{x}$ be the subalgebra of $\left(\operatorname{End}\left(S_{x}\right), \circ\right)$ consisting of those endomorphisms $T_{x} \in$ End $\left(S_{x}\right)$ which commute with any operator lying in the image of $\gamma_{x}$ :

$$
\begin{aligned}
\Sigma_{x} & \stackrel{\text { def }}{=}\left\{T_{x} \in \operatorname{End}\left(S_{x}\right) \mid\left[\gamma_{x}\left(\omega_{x}\right), T_{x}\right]_{-, o}=0, \forall \omega_{x}\right. \\
& \left.\in \wedge T_{x}^{*} M\right\} .
\end{aligned}
$$

Then $\Sigma_{x}$ is isomorphic with $\mathbb{S}_{\mathbb{K}}(p, q)$ for all $x \in M$. The bundle determined by $\Sigma_{x}$ when $x$ varies on $M$ will be called the Schur bundle of $\gamma$; it is a bundle of subalgebras of $(\operatorname{End}(S), \circ)$. Of course, the space $S_{x}$ can be viewed as a left $\Sigma_{x}$-module via the obvious action of the elements of $\Sigma_{x}-$ whereby $S$ can be viewed as a bundle of modules over the Schur bundle. The image $\gamma\left(\Lambda T_{\mathbb{K}}^{*} M\right)$ of $\gamma$ coincides with the subbundle $\operatorname{End}_{\Sigma}(S) \subset \operatorname{End}(S)$ whose fiber at $x \in M$ is given by

$$
\begin{aligned}
& \operatorname{End}_{\Sigma}(S)_{x} \\
& \quad=\left\{T_{x} \in \operatorname{End}(S) \mid\left[T_{x}, U_{x}\right]_{-, o}=0, \forall U \in \Sigma_{x}\right\},
\end{aligned}
$$

while its space of globally defined smooth sections is

$$
\begin{aligned}
& \Gamma\left(M, \operatorname{End}_{\Sigma}(S)\right)=\left\{T \in \Gamma(M, \operatorname{End}(S)) \mid[T, V]_{-, \mathrm{o}}\right. \\
& \quad=0, \forall V \in \Gamma(M, \Sigma)\} .
\end{aligned}
$$

We also note that the image of the map induced by $\gamma$ on sections is given by

$$
\gamma\left(\Omega_{\mathbb{K}}(M)\right)=\Gamma\left(M, \operatorname{End}_{\Sigma}(S)\right) .
$$

Irreducible Algebra Representations of the Fiber of the KählerAtiyah Bundle. We end by recalling some well-known facts from the representation theory of Clifford algebras: (i) A simple Clifford algebra admits (up to $\mathbb{K}$-linear equivalence) a single nontrivial irreducible representation by $\mathbb{K}$-linear operators, whose dimension equals $\Delta_{\mathbb{K}}(d) \operatorname{dim}_{\mathbb{K}} \mathbb{S}_{\mathbb{K}}(p, q)$.

(ii) A nonsimple Clifford algebra admits (up to $\mathbb{K}$-linear equivalence) two nontrivial irreducible representations by $\mathbb{K}$-linear operators, whose real dimensions are both equal to $\Delta_{\mathbb{K}}(d) \operatorname{dim}_{\mathbb{R}} \mathbb{S}_{\mathbb{K}}(p, q)$. The two representations map the Clifford volume element determined by some given orientation into a sign factor times the identity operator of the representation space and are distinguished from one another by the value of that signed factor.

4.3. A Partial Inverse of $\gamma$ in the Nonsimple Case. In the nonsimple case, the bundle morphism $\gamma$ has the property:

$$
\gamma(\nu)=\epsilon_{\gamma} \operatorname{id}_{S}
$$

where $\epsilon_{\gamma} \in\{-1,1\}$ is a sign factor which we will call the signature of $\gamma$. Direct computation using (161) gives

$$
\begin{gathered}
\gamma \circ P_{\epsilon_{\gamma}}=\gamma, \\
\gamma \circ P_{-\epsilon_{\gamma}}=0,
\end{gathered}
$$

which implies that $\gamma$ vanishes when restricted to the subbundle $\wedge^{-\epsilon_{\nu}} T_{\mathbb{K}}^{*} M$ and that its restriction to $\wedge^{\epsilon_{\nu}} T_{\mathbb{K}}^{*} M$ gives an isomorphism between this latter subbundle of algebras and the subbundle of algebras $\left(\operatorname{End}_{\Sigma}(S), \circ\right)$ of $\operatorname{End}(S)$. We have $\operatorname{ker}(\gamma)=\wedge^{-\epsilon_{\gamma}} T_{\mathbb{K}}^{*} M$. Hence the corresponding map on sections (which we denote again by $\gamma$ ) has kernel $\mathscr{K}(\gamma)=\Omega_{\mathbb{K}}^{-\epsilon_{\gamma}}(M)$ while its restriction to $\Omega_{\mathbb{K}}^{\epsilon_{\gamma}}(M)$ gives an isomorphism between this latter subalgebra of the Kähler-Atiyah algebra and the subalgebra $\Gamma\left(M, \operatorname{End}_{\Sigma}(S)\right)$ of $(\Gamma(M, \operatorname{End}(S)), \circ)$.

The Subbundle $\wedge^{\gamma} T_{\mathbb{K}}^{*} M$ and the Subalgebra $\Omega_{\mathbb{K}}^{\gamma}(M)$. Let us introduce notation which will allow us to treat all cases uniformly:

(i) In the nonsimple case (when the signature $\epsilon_{\gamma}$ is defined), we let $\wedge^{\gamma} T_{\mathbb{K}}^{*} M \stackrel{\text { def }}{=} \wedge^{\epsilon_{\nu}} T_{\mathbb{K}}^{*} M$ and $\Omega_{\mathbb{K}}^{\gamma}(M) \stackrel{\text { def }}{=}$ $\Omega_{\mathbb{K}}^{\epsilon_{\gamma}}(M)$.

(ii) In the simple case, we let $\wedge^{\gamma} T_{\mathbb{K}}^{*} M \stackrel{\text { def }}{=} \wedge T_{\mathbb{K}}^{*} M$ and $\Omega_{\mathbb{K}}^{\gamma}(M) \stackrel{\text { def }}{=} \Omega_{\mathbb{K}}(M)$.

In both cases, we have $\Omega_{\mathbb{K}}^{\gamma}(M)=\Gamma\left(M, \wedge^{\gamma} T_{\mathbb{K}}^{*} M\right)$. Notice that $\wedge^{\gamma} T_{\mathbb{K}}^{*} M$ is always a subbundle of unital algebras of the KählerAtiyah bundle while $\Omega_{\mathbb{K}}^{\gamma}(M)$ is always a unital subalgebra of the Kähler-Atiyah algebra.

The Partial Inverse of $\gamma$. Consider the bundle isomorphism:

$$
\left.\gamma\right|_{\wedge T_{\mathbb{K}}^{*} M} ^{\operatorname{End}_{\Sigma}(S)}: \wedge^{\gamma} T_{\mathbb{K}}^{*} M \stackrel{\sim}{\longrightarrow} \operatorname{End}_{\Sigma}(S)
$$


obtained by restricting the domain of definition of $\gamma$ to the subbundle $\wedge^{\gamma} T_{\mathbb{K}}^{*} M$ of the exterior bundle and the codomain of definition to the subbundle $\operatorname{End}_{\Sigma}(S)$ of $\operatorname{End}(S)$. We let

$$
\gamma^{-1} \stackrel{\text { def }}{=}\left(\left.\gamma\right|_{\wedge \gamma T_{\mathbb{K}}^{*} M} ^{\operatorname{End}_{\Sigma}(S)}\right)^{-1}: \operatorname{End}_{\Sigma}(S) \stackrel{\sim}{\longrightarrow} \wedge^{\gamma} T_{\mathbb{K}}^{*} M
$$

be the inverse of (163). The corresponding maps on sections give the mutually inverse isomorphisms of algebras displayed in the following diagram:

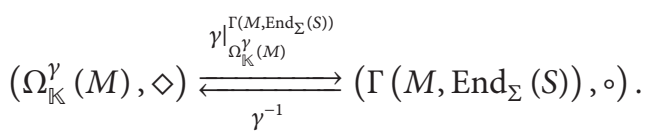

Notation 2. We define

$$
\check{T} \stackrel{\text { def }}{=} \gamma^{-1}(T) \in \Omega_{\mathbb{K}}^{\gamma}(M), \quad \forall T \in \Gamma\left(M, \operatorname{End}_{\Sigma}(S)\right) .
$$

In the context of "vertical quantization" of spin systems, $\gamma^{-1}$ plays the role of a (partial) "vertical dequantization map," so $\check{T}$ in (166) is the dequantization of a "vertical" operator $T$ acting in the Hilbert space. The partial inverse of $\gamma$ allows us to transfer statements about operators acting on pinors to statements about differential forms-an observation which will be used intensively in what follows. Notice the relations:

$$
\begin{aligned}
& \gamma^{-1} \circ \gamma=P_{\epsilon_{\gamma}}, \\
& \gamma \circ \gamma^{-1}=\operatorname{id}_{\operatorname{End}_{\Sigma}(S)}, \\
& \gamma \circ P_{\epsilon_{\gamma}}=\gamma, \\
& \gamma \circ P_{-\epsilon_{\gamma}}=0 .
\end{aligned}
$$

Local Expression for $\gamma^{-1}$. Considering a local pseudoorthonormal coframe $e^{a}$ and recalling that $\gamma\left(e^{a}\right)=\gamma^{a}$, we find

$$
\gamma^{-1}\left(\gamma^{a}\right)=e_{\gamma}^{a}
$$

where we have set

$$
e_{\gamma}^{a}= \begin{cases}e_{\epsilon_{\gamma}}^{a}, & \text { if we are in the non-simple case } \\ e^{a}, & \text { if we are in the simple case }\end{cases}
$$

with $e_{\epsilon_{\gamma}}^{a}$ defined as in (108). Relation (168) implies

$$
\begin{aligned}
& \gamma^{-1}\left(\gamma^{a_{1} \cdots a_{k}}\right) \\
& =e_{\gamma}^{a_{1} \cdots a_{k}} \begin{cases}e_{\epsilon_{\gamma}}^{a_{1} \cdots a_{k}}, & \text { if we are in the non-simple case } \\
e^{a_{1} \cdots a_{k}}, & \text { if we are in the simple case, }\end{cases}
\end{aligned}
$$

where $e_{ \pm}^{a_{1} \cdots a_{k}}$ are defined in (109) and we used the fact that $\gamma^{a_{1} \cdots a_{k}}=\gamma^{a_{1}} \circ \cdots \circ \gamma^{a_{k}}$ for all mutually distinct $a_{1} \cdots a_{k}$, the fact that $\gamma^{-1}$ is an isomorphism of algebras when corestricted to its image and (for the nonsimple case) identity (109).
4.4. Trace on $\Omega_{\mathbb{K}}^{\gamma}(M)$. The subalgebra $\Omega_{\mathbb{K}}^{\gamma}(M)$ admits a $\mathscr{C}^{\infty}(M, \mathbb{R})$-linear map $\mathcal{S}: \Omega_{\mathbb{K}}^{\epsilon}(M) \rightarrow \mathscr{C}^{\infty}(M, \mathbb{K})$ given by

$$
\mathcal{S}(\omega)=\omega^{(0)} N_{p, q} \mathrm{rk}_{\mathbb{K}}(S),
$$

where $\omega^{(0)}$ is the rank 0 component of $\omega$ (see expansion (2)), $S$ is any of the $\mathbb{K}$-pinor bundles, and $N_{p, q}$ equals 1 or 2 according to whether the corresponding fiberwise representation is faithful or not (notice that $N_{p, q} \mathrm{rk}_{\mathbb{K}} S$ is the dimension of the smallest faithful representation of the fiberwise Clifford algebra). One has

$$
\mathcal{S}(\omega)=\operatorname{tr}(\gamma(\omega)), \quad \forall \omega \in \Omega_{\mathbb{K}}^{\gamma}(M)
$$

as well as

$$
\begin{aligned}
\mathcal{S}\left(1_{M}\right) & =N_{p, q} \operatorname{dim}_{\mathbb{K}}(S), \\
\mathcal{S}(\omega \diamond \eta) & =\mathcal{S}(\eta \diamond \omega), \quad \forall \omega, \eta \in \Omega_{\mathbb{K}}^{\gamma}(M) .
\end{aligned}
$$

\section{The Fierz Isomorphism and Generalized Killing Forms}

In this section, we take up the issue of translating constrained generalized Killing pinor equations into conditions on differential forms. To simplify presentation, we will assume from the outset that the Schur algebra is isomorphic with the base field $\mathbb{K}$, so that either $\mathbb{K}=\mathbb{C}$ or we are in the normal case with $\mathbb{K}=\mathbb{R}$. We start in Section 5.1 with a discussion of the bundle of bipinors. Section 5.2 considers a certain isomorphism of bundles of algebras (which we will call the Fierz isomorphism) that provides an identification of the bundle of bipinors with the bundle $\left(\wedge^{\gamma} T_{\mathbb{K}}^{*} M, \diamond\right)$ and allows for a concise description of those Fierz identities which involve four pinors. This construction makes essential use of a choice of bilinear and nondegenerate "admissible" form $\mathscr{B}$ on the pin bundle (such inner products were classified in [30], see also [31]). Section 5.3 extracts some basic properties of this isomorphism which will be useful later on. In Section 5.4, we give a brief discussion of completeness relations for the endomorphism algebra of the pin bundle. Section 5.5 gives an explicit local expansion of the Fierz isomorphism which depends on the choice of a local pseudoorthonormal coframe. In Section 5.6, we show how algebraic constraints on pinors translate very directly into constraints on differential forms if one uses the basic properties of the Fierz isomorphism. Section 5.7 takes up the problem of translating generalized Killing pinor equations into conditions on differential forms. Using the Fierz isomorphism, we show that any connection on the pin bundle which is compatible with $\mathscr{B}$ defines a certain algebra connection on the KählerAtiyah bundle (i.e., a linear connection which is a fiberwise derivation of the geometric product) such that the Fierz isomorphism is flat with respect to the connections induced on its domain and codomain. Using this property, we show how one can easily translate generalized Killing conditions on pinors into differential constraints on forms defined on $M$. Section 5.8 gives another form of such differential constraints, which is used in Appendix B for comparison with 
the component approach outlined in [3]. In Section 5.9, we discuss some basic aspects of the algebrodifferential system of constraints on inhomogeneous forms which results from our analysis. As expected, our formulation allows one to extract basic structural properties of this system, thereby providing a starting point for a natural generalization of the classical theory of Killing forms. Finally, Section 5.10 considers the particular cases of one and two independent constrained generalized Killing pinors with a definite and symmetric $\operatorname{Spin}(d)$-invariant metric, the first of which is relevant to the application discussed in Section 6.

5.1. Bipinor Algebras. Let $S$ be a pin bundle over $(M, g)$ with underlying morphism $\gamma:\left(\wedge T_{\mathbb{K}}^{*} M, \diamond\right) \rightarrow(\operatorname{End}(S), \circ)$.

Admissible Bilinear Pairings on the Pin Bundle. It is well known that $S$ carries so-called admissible nondegenerate bilinear pairings $\mathscr{B}$ whose action on sections of $S$ satisfies

$$
\begin{aligned}
\mathscr{B}\left(\gamma(\omega) \xi, \xi^{\prime}\right) & =\mathscr{B}\left(\xi, \gamma\left(\tau_{\mathscr{B}}(\omega)\right) \xi^{\prime}\right), \\
\mathscr{B}\left(\xi^{\prime}, \xi\right)=\sigma_{\mathscr{B}} \mathscr{B}\left(\xi, \xi^{\prime}\right), & \\
& \forall \omega \in \Omega_{\mathbb{K}}(M), \forall \xi, \xi^{\prime} \in \Gamma(M, S)
\end{aligned}
$$

as well as another property which can be found in [31] but will not be relevant for what follows. In the formulas above, we used the following antiautomorphism of the Kähler-Atiyah algebra:

$$
\tau_{\mathscr{B}}=\pi^{\left(1-\epsilon_{\mathscr{B}}\right) / 2} \circ \tau= \begin{cases}\tau, & \text { if } \epsilon_{\mathscr{B}}=+1 \\ \pi \circ \tau, & \text { if } \epsilon_{\mathscr{B}}=-1,\end{cases}
$$

where $\tau$ is the reversion antiautomorphism defined in (41). The numbers $\epsilon_{\mathscr{B}}$ (the type of $\mathscr{B}$ ) and $\sigma_{\mathscr{B}}$ (the symmetry of $\mathscr{B})$ equal +1 or -1 , depending on $p, q$ and the precise choice of $\mathscr{B}$; such bilinear pairings were classified in [30, 31]. Notice that the first equation in (174) implies

$$
\gamma(\omega)^{t}=\gamma\left(\tau_{\mathscr{B}}(\omega)\right), \quad \forall \omega \in \Omega_{\mathbb{K}}(M) ;
$$

that is,

$$
()^{t} \circ \gamma=\gamma \circ \tau_{\mathscr{B}}
$$

where $T^{t}$ denotes the transpose of $T \in \Gamma(M, \operatorname{End}(S))$ with respect to $\mathscr{B}$, which is defined through

$$
\mathscr{B}\left(T \xi, \xi^{\prime}\right)=\mathscr{B}\left(\xi, T^{t} \xi^{\prime}\right), \quad \forall \xi, \xi^{\prime} \in \Gamma(M, S) .
$$

This operation satisfies $\left(T^{t}\right)^{t}=T$ and $\left(\mathrm{id}_{S}\right)^{t}=\mathrm{id}_{S}$, the first identity being a consequence of the signed symmetry property of $\mathscr{B}$ (the second identity listed in (174)). The operation $T \rightarrow T^{t}$ of taking the $\mathscr{B}$-transpose defines a $\mathscr{C}^{\infty}(M, \mathbb{R})$ linear antiautomorphism of the algebra $(\Gamma(M, \operatorname{End}(S)), \circ)$.

Local Expressions. Given a local pseudo-orthonormal coframe $e^{a}$ above $U \subset M$, the first of properties (174) amounts to

$$
\mathscr{B}\left(\gamma^{a} \xi, \xi^{\prime}\right)=\epsilon_{\mathscr{B}} \mathscr{B}\left(\xi, \gamma^{a} \xi^{\prime}\right), \quad \forall \xi, \xi^{\prime} \in \Gamma(U, S),
$$

which means that $\gamma^{a}=\gamma\left(e^{a}\right)$ satisfy $\left(\gamma^{a}\right)^{t}=\epsilon_{\mathscr{B}} \gamma^{a}$, a relation which implies

$$
\left(\gamma^{a_{1} \cdots a_{k}}\right)^{t}=\epsilon_{\mathscr{B}}^{k} \gamma^{a_{k} \cdots a_{1}},
$$

where $\epsilon_{\mathscr{B}}^{k}=\left(\epsilon_{\mathscr{B}}\right)^{k}$. Since $\left[\gamma^{a}, \gamma^{b}\right]_{+, \circ}=2 \eta^{a b}$, we also have

$$
\left(\gamma^{a}\right)^{-1}=\gamma_{a}, \quad \text { where } \gamma_{a} \stackrel{\text { def }}{=} \eta_{a b} \gamma^{b} \text {, }
$$

which in turn gives

$$
\left(\gamma^{a_{1} \cdots a_{k}}\right)^{-1}=\gamma_{a_{k} \cdots a_{1}}
$$

Combining the above, we find

$$
\left(\left(\gamma^{a_{1} \cdots a_{k}}\right)^{-1}\right)^{t}=\left(\gamma_{a_{k} \cdots a_{1}}\right)^{t}=\epsilon_{\mathscr{B}}^{k} \gamma_{a_{1} \cdots a_{k}},
$$

which implies

$$
\mathscr{B}\left(\left(\gamma^{a_{1} \cdots a_{k}}\right)^{-1} \xi, \xi^{\prime}\right)=\epsilon_{\mathscr{B}}^{k} \mathscr{B}\left(\xi, \gamma_{a_{1} \cdots a_{k}} \xi^{\prime}\right) .
$$

These relations will be useful later.

The Isomorphism E. The nondegenerate pairing $\mathscr{B}$ induces a bundle isomorphism $\rho: S \stackrel{\sim}{\rightarrow} S^{*}$, whose action on sections is given by

$$
\rho(\xi)\left(\xi^{\prime}\right) \stackrel{\text { def }}{=} \mathscr{B}\left(\xi^{\prime}, \xi\right), \quad \forall \xi, \xi^{\prime} \in \Gamma(M, S) .
$$

On the other hand, we have a natural bundle isomorphism $q: S \otimes S^{*} \stackrel{\sim}{\rightarrow} \operatorname{End}(S)$, given on sections as follows:

$$
\begin{aligned}
q(\xi \otimes \eta)\left(\xi^{\prime}\right) \stackrel{\text { def }}{=} & \eta\left(\xi^{\prime}\right) \xi \\
& \forall \xi, \xi^{\prime} \in \Gamma(M, S), \forall \eta \in \Gamma\left(M, S^{*}\right) .
\end{aligned}
$$

The two maps above combine to give a bundle isomorphism $E \stackrel{\text { def }}{=} q \circ\left(\operatorname{id}_{S} \otimes \rho\right): S \otimes S \stackrel{\sim}{\rightarrow} \operatorname{End}(S)$. Setting $E_{\xi, \xi^{\prime}} \stackrel{\text { def }}{=} E\left(\xi \otimes \xi^{\prime}\right) \epsilon$ $\Gamma(M, \operatorname{End}(S))$ for all $\xi, \xi^{\prime} \in \Gamma(M, S)$, we have

$$
\begin{aligned}
E_{\xi_{1}, \xi_{2}} \circ E_{\xi_{3}, \xi_{4}}=\mathscr{B}\left(\xi_{3}, \xi_{2}\right) & E_{\xi_{1}, \xi_{4}}, \\
& \forall \xi_{1}, \xi_{2}, \xi_{3}, \xi_{4} \in \Gamma(M, S),
\end{aligned}
$$

an identity which follows from the explicit form:

$$
E_{\xi, \xi^{\prime}}\left(\xi^{\prime \prime}\right)=\mathscr{B}\left(\xi^{\prime \prime}, \xi^{\prime}\right) \xi
$$

without making use of the signed symmetry property of $\mathscr{B}$. Note that $E$ depends on the choice of $\mathscr{B}$ (since $\rho$ does).

The Bundle of Bipinors and the Bipinor Algebra of $S$. The bundle isomorphism $E$ allows us to transfer the fiberwise composition of operators from $\operatorname{End}(S)$ to an associative and bilinear fiberwise composition - defined on the bundle of bipinors $S \otimes S$, whose action on sections takes the form

$$
u \cdot v \stackrel{\text { def }}{=} E^{-1}(E(u) \circ E(v)), \quad \forall u, v \in \Gamma(M, S \otimes S) .
$$


This operation satisfies

$$
\begin{aligned}
&\left(\xi_{1} \otimes \xi_{2}\right) \cdot\left(\xi_{3} \otimes \xi_{4}\right)=\mathscr{B}\left(\xi_{3}, \xi_{2}\right) \xi_{1} \otimes \xi_{4}, \\
& \forall \xi_{1}, \xi_{2}, \xi_{3}, \xi_{4} \in \Gamma(M, S) .
\end{aligned}
$$

The composition $\bullet$ makes the bundle of bipinors into a bundle of unital associative algebras which is isomorphic with the bundle of algebras $(\operatorname{End}(S), \circ)$; of course, the unit section $\mathrm{id}_{S}$ of $\operatorname{End}(S)$ maps to the unit section of $S \otimes S$, which we denote by $\mathscr{I} \stackrel{\text { def }}{=} E^{-1}\left(\mathrm{id}_{S}\right)$. The unital associative algebra $\Gamma(M, S \otimes$ $S)=\Gamma(M, S) \otimes_{\mathscr{C}^{\infty}(M, \mathbb{R})} \Gamma(M, S)$ consisting of smooth sections of the bipinor bundle will be called the bipinor algebra of $S$; it is an algebra over the ring $\mathscr{C}^{\infty}(M, \mathbb{R})$.

The Bipinor $\mathscr{C}^{\infty}(M, \mathbb{R})$-Algebra of a Submodule of Sections. If $\mathscr{K}$ is any $\mathbb{K}$-linear subspace of $\Gamma(M, S)$, then the set

$$
\begin{aligned}
& \mathscr{K} \otimes_{\mathscr{C}^{\infty}(M, \mathbb{R})} \mathscr{K} \stackrel{\text { def }}{=}\left\{\xi \otimes \xi^{\prime} \mid \xi, \xi^{\prime} \in \mathscr{K}\right\} \\
& \quad \subset \Gamma(M, S \otimes S) \approx \Gamma(M, S) \otimes_{\mathscr{C}^{\infty}(M, \mathbb{R})} \Gamma(M, S)
\end{aligned}
$$

is a $\mathbb{K}$-linear subspace of $\Gamma(M, S \otimes S)$.

When $\mathscr{K} \subset \Gamma(M, S)$ is a submodule of the $\mathscr{C}^{\infty}(M, \mathbb{R})$ module $\Gamma(M, S)$, then the subspace $\mathscr{K} \otimes_{\mathscr{C}^{\infty}(M, \mathbb{R})} \mathscr{K} \subset$ $\Gamma(M, S \otimes S)$ is a (generally nonunital) subalgebra of the bipinor algebra of $S$, which we will call the bipinor algebra of $\mathscr{K}$. This associative algebra defined over $\mathscr{C}^{\infty}(M, \mathbb{R})$ depends on the choice of $\mathscr{B}$. In particular, the space of smooth global sections $\Gamma(M, K)$ of any vector subbundle $K$ of $S$ is a $\mathscr{C}^{\infty}(M, \mathbb{R})$ submodule of $\Gamma(M, S)$ and the corresponding bipinor algebra $\Gamma(M, K) \otimes_{\mathscr{C}^{\infty}(M, \mathbb{R})} \Gamma(M, K)=\Gamma(M, K \otimes K)$ will be called the bipinor algebra of $K$.

The Bipinor $\mathbb{K}$-Algebra of a $\mathscr{B}$-Flat Subspace of Sections. Another interesting case arises when the $\mathbb{K}$-linear subspace $\mathscr{K} \subset \Gamma(M, S)$ is $\mathscr{B}$-flat, by which we mean that $\mathscr{K}$ satisfies the condition:

$$
\mathscr{B}\left(\xi, \xi^{\prime}\right) \text { is a constant function on } M \text {, }
$$

$$
\forall \xi, \xi^{\prime} \in \mathscr{K} \text {. }
$$

In this case, $\mathscr{K} \otimes_{\mathscr{C}^{\infty}(M, \mathbb{R})} \mathscr{K}$ is a $\mathbb{K}$-subalgebra of the bipinor algebra $(\Gamma(M, S \otimes S), \bullet)$, which we will call the (flat) bipinor $\mathbb{K}$-algebra determined by $\mathscr{K}$.

\subsection{The Fierz Isomorphism and Fierz Identities: Fierz Algebras}

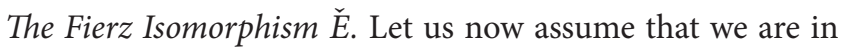
one of the cases when the Schur algebra is isomorphic with the base field. Then one can also transport to the bundle of bipinors the isomorphism $\gamma^{-1}: \operatorname{End}(S) \stackrel{\sim}{\rightarrow}\left(\wedge T_{\mathbb{K}}^{*} M\right)^{\gamma}$ to get an isomorphism of bundles of algebras:

$$
\check{E} \stackrel{\text { def }}{=} \gamma^{-1} \circ E:(S \otimes S, \bullet) \stackrel{\sim}{\longrightarrow}\left(\wedge^{\gamma} T_{\mathbb{K}}^{*} M, \diamond\right),
$$

which we will call the Fierz isomorphism. On sections, this induces a $\mathscr{C}^{\infty}(M, \mathbb{R})$-linear isomorphism of algebras (denoted, as usual, by the same symbol):

$$
\check{E} \stackrel{\text { def }}{=} \gamma^{-1} \circ E:(\Gamma(M, S \otimes S), \bullet) \stackrel{\sim}{\longrightarrow}\left(\Omega_{\mathbb{K}}^{\gamma}(M), \diamond\right),
$$

which identifies the bipinor algebra with the subalgebra $\Omega_{\mathbb{K}}^{\gamma}(M)$ of the Kähler-Atiyah algebra. Note that $\check{E}$ depends on the choice of admissible form $\mathscr{B}$.

Fierz Identities Involving Four Pinors. Setting $\check{E}_{\xi, \xi^{\prime}} \stackrel{\text { def }}{=} \check{E}(\xi \otimes$ $\left.\xi^{\prime}\right)=\gamma^{-1}\left(E_{\xi, \xi^{\prime}}\right) \in \Omega_{\mathbb{K}}^{\gamma}(M)$ (for $\xi, \xi^{\prime} \in \Gamma(M, S)$ ), (187) implies the following identity in the subalgebra $\Omega_{\mathbb{K}}^{\gamma}(M)$ of the KählerAtiyah algebra:

$$
\begin{aligned}
\check{E}_{\xi_{1}, \xi_{2}} \diamond \check{E}_{\xi_{3}, \xi_{4}}=\mathscr{B}\left(\xi_{3}, \xi_{2}\right) & \check{E}_{\xi_{1}, \xi_{4}}, \\
& \forall \xi_{1}, \xi_{2}, \xi_{3}, \xi_{4} \in \Gamma(M, S) .
\end{aligned}
$$

Equation (195) is the condensed expression of Fierz identities involving four pinors. These identities simply express the fact that $\gamma$ (and thus $\check{E}$ ) is an isomorphism of bundles of algebras, rather than simply an isomorphism of vector bundles-and are, in fact, equivalent to this property once fiberwise linearity of $\gamma$ is assumed. The construction of $\check{E}$ is summarized in the commutative diagram (196), which applies provided that $\mathbb{S}_{\mathbb{K}}(p, q) \approx \mathbb{K}$. In the diagram, we show the action of the various morphisms on sections. Consider

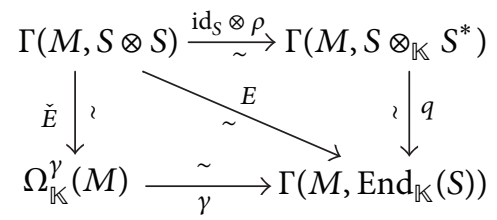

Notation 3. Let $\mathscr{K} \subset \Gamma(M, S)$ be any $\mathbb{K}$-linear subspace of $\Gamma(M, S)$. The image of the $\mathbb{K}$-linear subspace $\mathscr{K} \otimes_{\mathscr{C}^{\infty}(M, \mathbb{R})} \mathscr{K} \subset$ $\Gamma(M, S \otimes S)$ through the Fierz isomorphism will be denoted by

$$
\check{\mathscr{K}} \stackrel{\text { def }}{=} \check{E}\left(\mathscr{K} \otimes_{\mathscr{C}^{\infty}(M, \mathbb{R})} \mathscr{K}\right) \subset \Omega_{\mathbb{K}}^{\gamma}(M)
$$

and is a $\mathbb{K}$-linear subspace of $\Omega_{\mathbb{K}}^{\gamma}(M)$.

The Fierz $\mathscr{C}^{\infty}(M, \mathbb{R})$-Algebra of a Submodule of Sections. When the subspace $\mathscr{K} \subset \Gamma(M, S)$ is a submodule of the $\mathscr{C}^{\infty}(M, \mathbb{R})$-module $\Gamma(M, S)$, then the subspace $\mathscr{K} \otimes_{\mathscr{C}^{\infty}(M, \mathbb{R})} \mathscr{K} \subset \Gamma(M, S \otimes S)$ is a (generally nonunital) subalgebra (over $\mathscr{C}^{\infty}(M, \mathbb{R})$ ) of the bipinor algebra of $S$. Its image (197) through the Fierz isomorphism is a (generally nonunital) subalgebra of the $\mathscr{C}^{\infty}(M, \mathbb{R})$-algebra $\left(\Omega_{\mathbb{K}}^{\gamma}(M), \diamond\right)$, which we will call the Fierz subalgebra determined by $\mathscr{K}$. This algebra over $\mathscr{C}^{\infty}(M, \mathbb{R})$ encodes the Fierz identities between bilinears constructed from pinors which belong to $\mathscr{K}$. A particular case arises when $\mathscr{K}=\Gamma(M, K)$ where $K \subset S$ is some vector subbundle of $S$-in which situation $\mathscr{K} \otimes_{\mathscr{C}^{\infty}(M, \mathbb{R})} \mathscr{K}$ is the bipinor algebra of $K$. The corresponding Fierz subalgebra $\check{\mathscr{K}}$ will then be called the Fierz subalgebra determined by the subbundle $K$. With the further assumption that $\mathscr{B}$ is a scalar product (as happens in the application of Section 6), the morphism $E$ can then be used to identify the Fierz algebra of $K$ with the $\mathscr{C}^{\infty}(M, \mathbb{K})$-algebra $\Gamma(M, \operatorname{End}(K))$ 
of globally defined endomorphisms of the bundle $K$; in particular, the Fierz subalgebra is unital in such cases.

The Fierz $\mathbb{K}$-Algebra of a $\mathscr{B}$-Flat Subspace of Sections. When $\mathscr{K}$ is a $\mathscr{B}$-flat $\mathbb{K}$-linear subspace of $\Gamma(M, \mathbb{K})$, the vector space $\mathscr{K} \otimes_{\mathscr{C}^{\infty}(M, \mathbb{R})} \mathscr{K}$ is a $\mathbb{K}$-subalgebra of the bipinor algebra $(\Gamma(M, S \otimes S), \bullet)$. It follows that its image (197) through the Fierz isomorphism is a $\mathbb{K}$-subalgebra of the algebra $\left(\Omega_{\mathbb{K}}^{\gamma}(M), \diamond\right)$, which will be called the (flat) Fierz $\mathbb{K}$-algebra determined by $\mathscr{K}$.

5.3. Some Properties of the Fierz Isomorphism. A simple computation using (188) shows that the following identities hold for any $T \in \Gamma(M, \operatorname{End}(S))$ :

$$
\begin{aligned}
& T \circ E_{\xi, \xi^{\prime}}=E_{T \xi, \xi^{\prime}}, \\
& E_{\xi, \xi^{\prime}} \circ T=E_{\xi, T^{t} \xi^{\prime}},
\end{aligned}
$$

$$
\forall \xi, \xi^{\prime} \in \Gamma(M, S)
$$

that is,

$$
\begin{aligned}
& L_{T} \circ E=E \circ\left(T \otimes \mathrm{id}_{S}\right), \\
& R_{T} \circ E=E \circ\left(\mathrm{id}_{S} \otimes T^{t}\right),
\end{aligned}
$$

where $L_{T}$ and $R_{T}$ are the operators of left and right multiplication with $T$ in the algebra $(\Gamma(M, \operatorname{End}(S)), \circ)$. Applying $\gamma^{-1}$ to identities (198) and setting $\check{T} \stackrel{\text { def }}{=} \gamma^{-1}(T) \in \Omega_{\mathbb{K}}^{\gamma}(M)$ give

$$
\begin{aligned}
& \check{T} \diamond \check{E}_{\xi, \xi^{\prime}}=\check{E}_{T \xi, \xi^{\prime}}, \\
& \check{E}_{\xi, \xi^{\prime}} \diamond \check{T}=\check{E}_{\xi, T^{t} \xi^{\prime}},
\end{aligned}
$$

that is, (substituting $T \rightarrow T^{t}$ into the second equation)

$$
\begin{array}{r}
\check{T} \diamond \check{E}_{\xi, \xi^{\prime}}=\check{E}_{T \xi, \xi^{\prime}}, \\
\check{E}_{\xi, \xi^{\prime}} \diamond\left(T^{t}\right)^{\check{ }}=\check{E}_{\xi, T \xi^{\prime}} \cdot
\end{array}
$$

This also reads

$$
\begin{aligned}
& L_{\check{T}} \circ \check{E}=\check{E} \circ\left(T \otimes \mathrm{id}_{S}\right), \\
& R_{\check{T}} \circ \check{E}=\check{E} \circ\left(\mathrm{id}_{S} \otimes T^{t}\right),
\end{aligned}
$$

where $L_{\check{T}}$ and $R_{\check{T}}$ are the operators of left and right multiplication with $\check{T}$ in the Kähler-Atiyah algebra. Equation (177) implies $\gamma^{-1} \circ()^{t}=\tau_{\mathscr{B}} \circ \gamma^{-1}$; that is,

$$
\begin{aligned}
\gamma^{-1}\left(T^{t}\right)=\tau_{\mathscr{B}}\left(\gamma^{-1}(T)\right) \Longleftrightarrow & \left(T^{t}\right)^{\check{ }}=\tau_{\mathscr{B}}(\check{T}), \\
& \forall T \in \Gamma(M, \text { End }(S)) .
\end{aligned}
$$

We can thus write the second relation of (200) in the form

$$
\begin{aligned}
\check{E}_{\xi, \xi^{\prime}} \diamond \tau_{\mathscr{B}}(\check{T}) & =\check{E}_{\xi, T \xi^{\prime}}, \\
& \forall T \in \Gamma(M, \text { End }(S)), \forall \xi, \xi^{\prime} \in \Gamma(M, S) .
\end{aligned}
$$

We also notice the relation:

$$
\begin{aligned}
&\left(E_{\xi, \xi^{\prime}}\right)^{t}=\sigma_{\mathscr{B}} E_{\xi^{\prime}, \xi} \Longleftrightarrow \tau_{\mathscr{B}}\left(\check{E}_{\xi, \xi^{\prime}}\right)=\sigma_{\mathscr{B}} \check{E}_{\xi^{\prime}, \xi}, \\
& \forall \xi, \xi^{\prime} \in \Gamma(M, S),
\end{aligned}
$$

which follows from the signed symmetry of $\mathscr{B}$ together with definition (188). The last identity can also be written as follows:

$$
()^{t} \circ E=E \circ \operatorname{transp} p_{\mathscr{B}} \Longleftrightarrow \tau_{\mathscr{B}} \circ \check{E}=\check{E} \circ \operatorname{transp} p_{\mathscr{B}},
$$

where $\operatorname{transp}_{\mathscr{B}} \stackrel{\text { def }}{=} \sigma_{\mathscr{B}}$ transp and transp : $\Gamma(M, S \otimes S) \rightarrow$ $\Gamma(M, S \otimes S)$ is the $\mathscr{C}^{\infty}(M, \mathbb{R})$-linear operator which is defined as follows on decomposable elements:

$$
\operatorname{transp}\left(\xi \otimes \xi^{\prime}\right)=\xi^{\prime} \otimes \xi, \quad \forall \xi, \xi^{\prime} \in \Gamma(M, S) .
$$

One easily checks that transp $\mathrm{P}_{\mathscr{B}}$ is an antiautomorphism of the bipinor algebra. For later reference, note the identity:

$$
\begin{aligned}
\operatorname{tr} \circ L_{T} \circ E & =\mathscr{B} \circ\left(T \otimes \mathrm{id}_{S}\right) \Longleftrightarrow \\
\operatorname{tr}\left(T \circ E_{\xi, \xi^{\prime}}\right) & =\mathscr{B}\left(T \xi, \xi^{\prime}\right), \quad \forall \xi, \xi^{\prime} \in \Gamma(M, S),
\end{aligned}
$$

which will be useful below.

5.4. Local Completeness Relations for the Endomorphism Algebra of the Pin Bundle. Let tr : $\operatorname{End}(S) \rightarrow \mathcal{O}_{\mathbb{K}}$ be the natural ${ }^{6}$ trace on $\operatorname{End}(S)$ (which is a morphism of $\mathbb{K}$ vector bundles). Recall that we assume the Schur algebra to be isomorphic with the base field $\mathbb{K}$. Then a convenient generating set of local sections for the vector bundle $\operatorname{End}(S)$ above a sufficiently small open subset $U \subset M$ is given (see, e.g., [28]) by the operators $\left\{\gamma^{a_{1} \cdots a_{k}} \mid k=0, \ldots, d, 1 \leq a_{1}<\right.$ $\left.\cdots<a_{k} \leq d\right\}$, where $d=p+q$ and $\gamma^{a}=\gamma\left(e^{a}\right)$ with $\left(e^{a}\right)$ a pseudo-orthonormal local coframe of $M$ above $U$. The following identity (the "completeness relation") holds:

$$
T={ }_{U} \frac{\Delta_{\mathbb{R}}(d)}{2^{d}} \sum_{k=0}^{d} \frac{1}{k !} \operatorname{tr}\left(\gamma^{a_{k} \cdots a_{1}} \circ T\right) \gamma_{a_{1} \cdots a_{k}},
$$

$$
\forall T \in \Gamma(U, \text { End }(S)),
$$

where $\Delta_{\mathbb{R}}(d)=2^{[d / 2]}$ for $\mathbb{S} \approx \mathbb{R}$ or $\mathbb{S} \approx \mathbb{C}($ see Section 3.8).

Remark 10. The $\mathbb{K}$-vector bundles $\operatorname{End}(S) \otimes \operatorname{End}(S)$ and $\operatorname{End}(\operatorname{End}(S))$ can be identified through the bundle isomorphism $\mathscr{W}: \operatorname{End}(S) \otimes \operatorname{End}(S) \rightarrow \operatorname{End}(\operatorname{End}(S))$, which acts as follows on sections:

$\mathscr{W}(A \otimes B)(T)=A \operatorname{tr}(B \circ T)$,

$$
\forall A, B, T \in \Gamma(M, \text { End }(S)) .
$$

Using this isomorphism, we transport the composition 。 of $\operatorname{End}(\operatorname{End}(S))$ to an associative composition - defined on $\operatorname{End}(S) \otimes \operatorname{End}(S)$, whose action on sections is given by

$$
\begin{aligned}
&(A \otimes B) \bullet\left(A^{\prime} \otimes B^{\prime}\right) \\
&=\mathscr{W}^{-1}\left(\mathscr{W}(A \otimes B) \circ \mathscr{W}\left(A^{\prime} \otimes B^{\prime}\right)\right), \\
& \forall A, A^{\prime}, B, B^{\prime} \in \Gamma(M, \text { End }(S)) .
\end{aligned}
$$


An easy computation shows that • has the explicit form:

$$
\begin{aligned}
(A \otimes B) \bullet\left(A^{\prime} \otimes B^{\prime}\right)= & \operatorname{tr}\left(A^{\prime} \circ B\right) A \otimes B^{\prime}, \\
& \forall A, A^{\prime}, B, B^{\prime} \in \Gamma(M, \text { End }(S)) .
\end{aligned}
$$

The unit section $\mathrm{id}_{\operatorname{End}(S)}$ of the bundle $\operatorname{End}(\operatorname{End}(S))$ corresponds via $\mathscr{W}$ to the unit section $\mathscr{I} \stackrel{\text { def }}{=} \mathscr{W}^{-1}\left(\mathrm{id}_{\operatorname{End}(S)}\right)$ of $(\operatorname{End}(S) \otimes \operatorname{End}(S), \bullet)$. Expression (212) shows that the completeness relation is equivalent to the following decomposition of the unit $\left.\mathscr{I}\right|_{U}$ of the algebra $(\Gamma(U, \operatorname{End}(S) \otimes$ $\operatorname{End}(S)), \bullet)$ :

$$
\mathscr{I}=\sum_{U} \sum_{k=0}^{d} \frac{1}{k !} \gamma_{a_{1} \cdots a_{k}} \otimes \gamma^{a_{k} \cdots a_{1}}
$$

5.5. Explicit Expansion of the Fierz Isomorphism When the Schur Algebra Equals the Base Field. Given a local pseudoorthonormal coframe of $M$, explicit expansions for the isomorphism $\check{E}$ can be derived using the results of [28] (see also [32]) for any choices of the base field $\mathbb{K}$ and of the signature type $(p, q)$. A complete discussion is quite involved given the different behavior in various cases and will be taken up in detail in a different publication. Below, we will consider only the case when the Schur algebra is isomorphic with the base field $\mathbb{K}$, that is, the case when $\mathbb{K}=\mathbb{C}$ and the case when $\mathbb{K}=\mathbb{R}$ with $p-q \equiv_{8} 0,1,2$.

In this case, the local completeness relation (209) holds. Applying it to the endomorphism $T=E_{\xi, \xi^{\prime}}$ and using relation (208), we find the local expansion:

$$
\begin{array}{r}
E_{\xi, \xi^{\prime}}=\frac{1}{2^{[(d+1) / 2]}} \sum_{k=0}^{d} \frac{1}{k !} \mathscr{B}\left(\gamma_{a_{k} \cdots a_{1}} \xi, \xi^{\prime}\right) \gamma^{a_{1} \cdots a_{k}}, \\
\forall \xi, \xi^{\prime} \in \Gamma(U, S),
\end{array}
$$

which implies the following local expansion of the Fierz isomorphism upon applying $\gamma^{-1}$ to both sides:

$$
\check{E}_{\xi, \xi^{\prime}}=\frac{1}{2^{[(d+1) / 2]}} \check{\mathbf{E}}_{\xi, \xi^{\prime}}
$$

an identity holding in $\Omega_{\mathbb{K}}^{\gamma}(M)$, where

$$
\check{\mathbf{E}}_{\xi, \xi^{\prime}}=\sum_{k=0}^{d} \check{\mathbf{E}}_{\xi, \xi^{\prime}}^{(k)}
$$

as in (2), with

$$
\check{\mathbf{E}}_{\xi, \xi^{\prime}}^{(k)}={ }_{U} \frac{1}{k !} \check{\mathbf{E}}_{a_{1} \cdots a_{k}}^{(k)}\left(\xi, \xi^{\prime}\right) e_{\gamma}^{a_{1} \cdots a_{k}}
$$

where, using (184), we have

$$
\check{\mathbf{E}}_{a_{1} \cdots a_{k}}^{(k)}\left(\xi, \xi^{\prime}\right)=\mathscr{B}\left(\gamma_{a_{k} \cdots a_{1}} \xi, \xi^{\prime}\right)=\epsilon_{\mathscr{B}}^{k} \mathscr{B}\left(\xi, \gamma_{a_{1} \cdots a_{k}} \xi^{\prime}\right)
$$

5.6. Expressing the Algebraic Constraints through Differential Forms. Consider the case when we have a single algebraic constraint $Q \xi=0$, where $Q \in \Gamma(M, \operatorname{End}(S))$. As in Section 2, we let $\mathscr{K}(Q)$ denote the space of solutions of the algebraic constraint, which is a submodule of the $\mathscr{C}^{\infty}(M, \mathbb{K})$-module $\Gamma(M, S)$. Recall that there generally exists no subbundle of $S$ whose space of smooth sections equals $\mathscr{K}(Q)$.

The Dequantized Constraint and the $\mathscr{C}^{\infty}(M, \mathbb{R})$-Subalgebra of Constrained Inhomogeneous Forms. The inhomogeneous form

$$
\check{Q}=\gamma^{-1}(Q) \in \Omega_{\mathbb{K}}^{\gamma}(M)
$$

will be called the dequantization of Q. Relation (199) gives

$$
\begin{gathered}
L_{Q} \circ E=E \circ\left(\mathrm{id}_{S} \otimes Q\right), \\
R_{Q^{\ddagger}} \circ E=E \circ\left(Q \otimes \mathrm{id}_{S}\right),
\end{gathered}
$$

where $L_{Q}, R_{Q^{t}}$ are-as above-the operators on $\Gamma(M$, End $(S))$ given by left and right composition with $Q$ and $Q^{t}$. Using the fact that $E$ is an isomorphism as well as the identities $\mathscr{K}\left(Q \otimes \mathrm{id}_{S}\right)=\mathscr{K}(Q) \otimes_{\mathscr{C}^{\infty}(M, \mathbb{R})} \Gamma(M, S)$ and $\mathscr{K}\left(\mathrm{id}_{S} \otimes Q\right)=$ $\Gamma(M, S) \otimes_{\mathscr{C}^{\infty}(M, \mathbb{R})} \mathscr{K}(Q)$, we find $\mathscr{K}(Q) \otimes_{\mathscr{C}^{\infty}(M, \mathbb{R})} \mathscr{K}(Q)=$ $\mathscr{K}\left(Q \otimes \mathrm{id}_{S}\right) \cap \mathscr{K}\left(\mathrm{id}_{S} \otimes Q\right)$ and

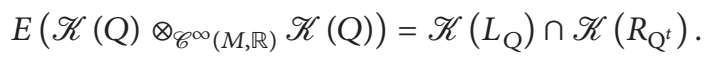

Applying $\gamma^{-1}$ to both sides of this relation gives the following description of the Fierz algebra of the submodule of sections $\mathscr{K}(Q)$, which we will call the $\mathscr{C}^{\infty}(M, \mathbb{R})$-algebra of $Q$ constrained inhomogeneous forms:

$$
\begin{aligned}
\check{\mathscr{K}}_{\mathrm{Q}} \stackrel{\text { def }}{=} \check{E}\left(\mathscr{K}(Q) \otimes_{\mathscr{C}^{\infty}(M, \mathbb{R})} \mathscr{K}(Q)\right) \\
=\mathscr{K}\left(L_{\check{Q}}\right) \cap \mathscr{K}\left(R_{\tau_{\mathscr{B}}(\check{Q})}\right) \cap \Omega_{\mathbb{K}}^{\gamma}(M) \\
=\mathscr{K}\left(L_{\check{Q}}+R_{\tau_{\mathscr{B}}(\check{Q})}\right) \cap \mathscr{K}\left(L_{\check{Q}}-R_{\tau_{\mathscr{B}}(\check{Q})}\right) \\
\cap \Omega_{\mathbb{K}}^{\gamma}(M) .
\end{aligned}
$$

Here, $L_{\check{Q}}, R_{\check{Q}}$ are the left and right $\diamond$-multiplication operators of Section 3.4 and the second equality above is obvious. With these definitions, we have the equivalence:

$$
\check{E}_{\xi, \xi^{\prime}} \in \check{\mathscr{K}}_{\mathrm{Q}} \Longleftrightarrow \xi \otimes \xi^{\prime} \in \mathscr{K}(Q) \otimes_{\mathscr{C}^{\infty}(M, \mathbb{R})} \mathscr{K}(Q) ;
$$

that is,

$$
\begin{aligned}
\check{Q} \diamond \check{E}_{\xi, \xi^{\prime}} & =\check{E}_{\xi, \xi^{\prime}} \diamond \tau_{\mathscr{B}}(\check{Q})=0 \Longleftrightarrow \\
\xi & =0 \\
& \text { or } \xi^{\prime}=0 \\
& \text { or } Q \xi=Q \xi^{\prime}=0 .
\end{aligned}
$$


Behavior under $\tau_{\mathscr{B}}$. The equivalence (224) can be written in the following form, which follows by applying $\tau_{\mathscr{B}}$ to the second equation in the left hand side

$$
\begin{gathered}
\check{Q} \diamond \check{E}_{\xi, \xi^{\prime}}=\check{Q} \diamond \tau_{\mathscr{B}}\left(\check{E}_{\xi, \xi^{\prime}}\right)=0 \Longleftrightarrow \\
\xi \otimes \xi^{\prime} \in \mathscr{K}(Q) \otimes_{\mathscr{C}^{\infty}(M, \mathbb{R})} \mathscr{K}(Q)
\end{gathered}
$$

or (using (205)) in the form

$$
\begin{aligned}
\check{Q} \diamond \check{E}_{\xi, \xi^{\prime}}=\check{Q} \diamond \check{E}_{\xi^{\prime}, \xi}=0 \Longleftrightarrow \\
\xi \otimes \xi^{\prime} \in \mathscr{K}(Q) \otimes_{\mathscr{C}^{\infty}(M, \mathbb{R})} \mathscr{K}(Q) .
\end{aligned}
$$

In fact, relation (206) and the obvious fact that $\mathscr{K}(Q) \otimes_{\mathscr{C}^{\infty}(M, \mathbb{R})} \mathscr{K}(Q)$ is invariant under the antiauto-

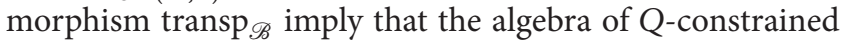
differential forms is invariant under $\tau_{\mathscr{B}}$ :

$$
\tau_{\mathscr{B}}\left(\check{\mathscr{K}}_{\mathrm{Q}}\right)=\check{\mathscr{K}}_{\mathrm{Q}} .
$$

$$
\left[\nabla_{m}^{S}, \gamma(\omega)\right]_{-, \circ}=\gamma\left(\nabla_{m} \omega\right), \quad \forall \omega \in \Omega_{\mathbb{K}}(M) \Longleftrightarrow\left(\nabla_{m}^{S}\right)^{\mathrm{ad}} \circ \gamma=\gamma \circ \nabla_{m} \Longleftrightarrow P_{\epsilon_{\gamma}} \circ \nabla_{m}=\gamma^{-1} \circ\left(\nabla_{m}^{S}\right)^{\mathrm{ad}} \circ \gamma,
$$

Remark 11. Using (204) and (215) and separating ranks shows that (224) can be written as follows:

$$
\begin{aligned}
& \xi \otimes \xi^{\prime} \in \mathscr{K}(Q) \otimes_{\mathscr{C}^{\infty}(M, \mathbb{R})} \mathscr{K}(Q) \Longleftrightarrow \\
& \check{\mathbf{E}}_{Q \xi, \xi^{\prime}}^{(k)}=\check{\mathbf{E}}_{\xi, Q \xi^{\prime}}^{(k)}=0, \quad \forall k=0 \cdots d,
\end{aligned}
$$

which amounts to the following description upon using the explicit form (218) of $\check{\mathbf{E}}$ :

$$
\begin{array}{r}
\xi \otimes \xi^{\prime} \in \mathscr{K}(Q) \otimes_{\mathscr{C}^{\infty}(M, \mathbb{R})} \mathscr{K}(Q) \Longleftrightarrow \\
\mathscr{B}\left(\xi, Q^{t} \circ \gamma_{a_{1} \cdots a_{k}} \xi^{\prime}\right)=\mathscr{B}\left(\xi, \gamma_{a_{1} \cdots a_{k}} \circ Q \xi^{\prime}\right)=0, \\
\forall \xi, \xi^{\prime} \in \mathscr{K}(Q) .
\end{array}
$$

In Appendix B.1, we show that (229) are equivalent to certain relations which were first discussed in [3] for the special case $d=p=8, q=0$.

5.7. Conditions on Differential Forms Implied by the Generalized Killing Equation. In our formulation, a Clifford connection $\nabla^{S}=\mathrm{d} x^{m} \otimes \nabla_{m}^{S}$ on $S$ is a connection which satisfies where $\nabla_{m}$ is the connection induced on $\wedge T_{\mathbb{K}}^{*} M$ by the LeviCivita connection of $(M, g)$ and $\left(\nabla_{m}^{S}\right)^{\text {ad }}: \Gamma(M, \operatorname{End}(S)) \rightarrow$ $\Gamma(M, \operatorname{End}(S))$ is the connection induced by $\nabla_{m}^{S}$ on $\operatorname{End}(S)$ :

$$
\begin{aligned}
&\left(\nabla_{m}^{S}\right)^{\mathrm{ad}}(T) \stackrel{\text { def }}{=}\left[\nabla_{m}^{S}, T\right]_{-, \circ}=\nabla_{m}^{S} \circ T-T \circ \nabla_{m}^{S}, \\
& \forall T \in \Gamma(M, \text { End }(S)) .
\end{aligned}
$$

Notice that $P_{\epsilon_{\gamma}} \circ \nabla$ is the connection induced by $\nabla$ on the subbundle $\left(\wedge T_{\mathbb{K}}^{*} M\right)^{\epsilon_{\gamma}}$ and that this induced connection is determined by $\left(\nabla^{S}\right)^{\text {ad }}$ through property (230). In the following, we take $\nabla^{S}$ to be the connection on $S$ induced by the Levi-Civita connection of $(M, g)$; it is well known that $\nabla^{S}$ is a Clifford connection in the sense discussed above ${ }^{7}$. A discussion of this and other properties of $\nabla^{S}$ in index language (which also serves to fix our conventions and leads to another derivation of certain identities extracted in this paper) can be found in Appendix A. Equation (230) is compatible with the fact that $\nabla_{m}$ is a derivation of the Kähler-Atiyah algebra $\left(\Omega_{\mathbb{K}}(M), \diamond\right)$-a property which can be checked by direct computation using the fact that $\nabla_{m}$ is an even derivation of the exterior algebra which is compatible with the metric. Similarly, we consider the connection $D^{\text {ad }}$ induced by $D$ on $\operatorname{End}(S)$ :

$$
D_{m}^{\mathrm{ad}}(T) \stackrel{\text { def }}{=} D_{m} \circ T-T \circ D_{m}
$$

Notation 4. We let $\mathscr{K}(D)=\bigcap_{m=1}^{d} \mathscr{K}\left(D_{m}\right) \subset \Gamma(M, S)$ be the finite-dimensional $\mathbb{K}$-linear subspace of all generalized Killing pinors with respect to $D$.

The Dequantized Connection. In the following, we consider only the case when the Schur algebra is isomorphic with the base field. Then any connection $D$ on $S$ can be written as follows:

$$
D_{m}=\nabla_{m}^{S}+A_{m}=\nabla_{m}^{S}+\gamma\left(\check{A}_{m}\right)
$$

where

$$
\check{A}_{m}=\gamma^{-1}\left(A_{m}\right) \in \Omega_{\mathbb{K}}^{\gamma}(M)
$$

are inhomogeneous differential forms on $M$. The Clifford connection property (230) of $\nabla^{S}$ implies

$$
\begin{aligned}
D_{m}^{\mathrm{ad}} \circ \gamma & =\gamma \circ \mathbf{D}_{m} \Longleftrightarrow \\
P_{\epsilon_{\gamma}} \circ \mathbf{D}_{m} & =\gamma^{-1} \circ D_{m}^{\mathrm{ad}} \circ \gamma \Longleftrightarrow \\
{\left[D_{m}, \gamma(\omega)\right]_{-, \circ} } & =\gamma\left(\mathbf{D}_{m} \omega\right), \quad \forall \omega \in \Omega_{\mathbb{K}}(M),
\end{aligned}
$$

where the derivation $\mathbf{D}_{m}$ (which we will call the adjoint dequantized connection) of the Kähler-Atiyah algebra $\left(\Omega_{\mathbb{K}}(M), \diamond\right)$ is defined through

$$
\mathbf{D}_{m} \omega \stackrel{\text { def }}{=} \nabla_{m} \omega+\left[\check{A}_{m}, \omega\right]_{-, \diamond}, \quad \forall \omega \in \Omega_{\mathbb{K}}(M) .
$$


Since $\nabla_{m} \nu=0$ and $\check{A}_{m} \in \Omega_{\mathbb{K}}^{\gamma}(M)$, we have $\left[\check{A}_{m}, \Omega_{\mathbb{K}}^{\gamma}(M)\right]_{-, \diamond} \subset$ $\Omega_{\mathbb{}}^{\gamma}(M)$ and $\mathbf{D}_{m}\left(\Omega_{\mathbb{K}}^{\gamma}(M)\right) \subset \Omega_{\mathbb{K}}^{\gamma}(M)$. Composing (235) with $\gamma^{-1}$ from both sides gives the following relation which will be used below:

$$
\mathbf{D}_{m} \circ \gamma^{-1}=\gamma^{-1} \circ D_{m}^{\mathrm{ad}} .
$$

To arrive at (237), we noticed that $\mathbf{D}_{m} \circ \gamma^{-1}\left(\operatorname{End}_{\mathbb{K}}(S)\right)=$ $D_{m}^{\mathrm{ad}}\left(\Omega_{\mathbb{K}}^{\gamma}(M)\right) \subset \Omega_{\mathbb{K}}^{\gamma}(M)$ implies $P_{\epsilon_{\gamma}} \circ \mathbf{D}_{m} \circ \gamma^{-1}=\mathbf{D}_{m} \circ \gamma^{-1}$, which in turn implies (237) upon using the equation $P_{\epsilon_{\gamma}} \circ \mathbf{D}_{m} \circ \gamma^{-1}=\gamma^{-1} \circ D_{m}^{\text {ad }}$, which follows upon composing the last equality in (235) with $\gamma^{-1}$ from the right and using the property $\gamma \circ \gamma^{-1}=\operatorname{id}_{\operatorname{End}_{K}(S)}$. Note that $A_{m}$ can be combined into the object:

$$
A=e^{m} \otimes A_{m} \in \Omega_{\mathbb{K}}^{1}(M) \otimes_{\mathscr{C}^{\infty}(M, \mathbb{R})} \Omega_{\mathbb{K}}^{\gamma}(M),
$$

while $\mathbf{D}_{m}$ can be combined into the map:

$$
\mathbf{D}=e^{m} \otimes \mathbf{D}_{m}=\nabla+e^{m} \otimes\left(\check{A}_{m}\right)^{\mathrm{ad}}: \Omega_{\mathbb{K}}(M) \longrightarrow \Omega_{\mathbb{K}}^{1}(M) \otimes_{\mathscr{C} \infty}(M, \mathbb{R}){ }_{\mathbb{K}}(M),
$$

where

$$
\left(\check{A}_{m}\right)^{\mathrm{ad}}(\omega):=\left[\check{A}_{m}, \omega\right]_{-, \diamond}
$$

and we used $e^{m} \otimes \nabla_{m}=\nabla$.

Flatness of the Fierz Isomorphism. For the remainder of this paper, we will assume that $D$ is compatible with $\mathscr{B}$ in the usual sense that $\mathscr{B}$ is $D$-flat:

$$
\begin{aligned}
\mathrm{d} \mathscr{B}\left(\xi, \xi^{\prime}\right) & =\mathscr{B}\left(D \xi, \xi^{\prime}\right)+\mathscr{B}\left(\xi, D \xi^{\prime}\right) \\
\partial_{m} \mathscr{B}\left(\xi, \xi^{\prime}\right)=\mathscr{B}\left(D_{m} \xi, \xi^{\prime}\right)+\mathscr{B}\left(\xi, D_{m} \xi^{\prime}\right), & \\
\forall \xi, \xi^{\prime} & \in \Gamma(M, S) .
\end{aligned}
$$

In this case, the $\mathbb{K}$-linear subspace $\mathscr{K}(D) \subset \Gamma(M, S)$ is $\mathscr{B}$-flat, so it defines a flat bipinor $\mathbb{K}$-algebra $\mathscr{K}(D) \otimes_{\mathscr{C} \infty(M, \mathbb{R})} \mathscr{K}(D) \subset$ $\Gamma(M, S \otimes S)$. Furthermore, the isomorphism $\rho: S \stackrel{\sim}{\rightarrow} S^{*}$ satisfies $D_{m}^{*} \circ \rho=\rho \circ D_{m}$ (where $D_{m}^{*}$ is the dual connection) while the natural isomorphism $q: S \otimes S^{*} \stackrel{\sim}{\rightarrow} \operatorname{End}(S)$ satisfies $D_{m}^{\mathrm{ad}} \circ q=q \circ\left(D_{m} \otimes \mathrm{id}_{S}+\mathrm{id}_{S} \otimes D_{m}^{*}\right)$. It follows that the isomorphism $E=q \circ\left(\operatorname{id}_{S} \otimes \rho\right)$ satisfies ${ }^{8}$

$$
D_{m}^{\mathrm{ad}} \circ E=E \circ\left(D_{m} \otimes \mathrm{id}_{S}+\mathrm{id}_{S} \otimes D_{m}\right) .
$$

In particular, we have

$$
E\left(\mathscr{K}(D) \otimes_{\mathscr{C} \infty}(M, \mathbb{R}) \mathscr{K}(D)\right) \subset \mathscr{K}\left(D^{\mathrm{ad}}\right),
$$

where we have introduced the following $\mathbb{K}$-subalgebra of $(\Gamma(M, \operatorname{End}(S)), \circ)$ :

$$
\mathscr{K}\left(D^{\text {ad }}\right) \stackrel{\text { def }}{=} \bigcap_{m=1}^{d} \mathscr{K}\left(D_{m}^{\text {ad }}\right) .
$$

On the other hand, $\gamma^{-1}$ satisfies (237). Together with (242), this implies that the Fierz isomorphism $\breve{E}$ satisfies

$$
\mathbf{D}_{m} \circ \check{E}=\check{E} \circ\left(D_{m} \otimes \mathrm{id}_{S}+\mathrm{id}_{S} \otimes D_{m}\right) .
$$

Therefore, we find

$$
\mathbf{D}_{m} \check{E}_{\xi, \xi^{\prime}}=\check{E}_{D_{m} \xi, \xi^{\prime}}+\check{E}_{\xi, D_{m} \xi^{\prime}}, \quad \forall \xi, \xi^{\prime} \in \Gamma(M, S) .
$$

The $\mathbb{K}$-Algebra of Generalized Killing Forms. We define the $\mathbb{K}$-algebra of generalized Killing forms to be the following $\mathbb{K}$ subalgebra of $\left(\Omega_{\nVdash}^{\gamma}(M), \diamond\right)$ :

$$
\check{\mathscr{K}}_{D} \stackrel{\text { def }}{=} \mathscr{K}(\mathbf{D}) \cap \Omega_{\mathbb{K}}^{\gamma}(M)=\gamma^{-1}\left(\mathscr{K}\left(D^{\text {ad }}\right)\right) .
$$

The elements of $\check{\mathscr{K}}_{D^{\text {ad }}}$ will be called generalized Killing forms. Relation (243) implies that the flat Fierz $\mathbb{K}$-algebra

$$
\check{\mathscr{K}}(D) \stackrel{\text { def }}{=} \check{E}\left(\mathscr{K}(D) \otimes_{\mathscr{C}^{\infty}(M, \mathbb{R})} \mathscr{K}(D)\right)
$$

defined by the $\mathscr{B}$-flat subspace $\mathscr{K}(D) \subset \Gamma(M, S)$ is a subalgebra of the $\mathbb{K}$-algebra of generalized Killing forms:

$$
\check{\mathscr{K}}(D) \subset \check{\mathscr{K}}_{D} .
$$

In particular, we have

$$
\mathbf{D}_{m} \check{E}_{\xi, \xi^{\prime}}=0, \quad \forall \xi, \xi^{\prime} \in \mathscr{K}(D) .
$$

Behavior under $\tau_{\mathscr{B}}$. Spin $(d)$-invariance of $\mathscr{B}$ implies that $\mathscr{B}$ is flat with respect to the connection $\nabla^{S}$ :

$$
\begin{aligned}
& \mathrm{d} \mathscr{B}\left(\xi, \xi^{\prime}\right)=\mathscr{B}\left(\nabla^{S} \xi, \xi^{\prime}\right)+\mathscr{B}\left(\xi, \nabla^{S} \xi^{\prime}\right) \\
& \partial_{m} \mathscr{B}\left(\xi, \xi^{\prime}\right)=\mathscr{B}\left(\nabla_{m}^{S} \xi, \xi^{\prime}\right)+\mathscr{B}\left(\xi, \nabla_{m}^{S} \xi^{\prime}\right), \\
& \forall \xi, \xi^{\prime} \in \Gamma(M, S),
\end{aligned}
$$

which is easily seen to imply the property:

$$
\left[\left(\nabla_{m}^{S}\right)^{\mathrm{ad}}(T)\right]^{t}=\left(\nabla_{m}^{S}\right)^{\mathrm{ad}}\left(T^{t}\right),
$$

$\forall T \in \Gamma(M$, End $(S))$.

Together with assumption (241), identity (251) implies that $A_{m}$ are $\mathscr{B}$-antisymmetric endomorphisms of $S$ :

$$
\begin{aligned}
A_{m}^{t}=-A_{m} \Longleftrightarrow \mathscr{B}\left(A_{m} \xi, \xi^{\prime}\right)=-\mathscr{B}\left(\xi, A_{m} \xi^{\prime}\right), \\
\forall \xi, \xi^{\prime} \in \Gamma(M, S) .
\end{aligned}
$$


In turn, these properties imply the relation $\left[A_{m}, T\right]_{-, \circ}^{t}=$ $\left[A_{m}, T^{t}\right]_{-, o}$, so $D_{m}^{\text {ad }}$ satisfies

$$
\begin{aligned}
D_{m}^{\mathrm{ad}}\left(T^{t}\right) & =\left(D_{m}^{\mathrm{ad}}(T)\right)^{t}, \\
\forall T & \in \Gamma(M, \text { End }(S)) \Longleftrightarrow D_{m}^{\mathrm{ad}} \circ()^{t}=()^{t} \circ D_{m}^{\mathrm{ad}} .
\end{aligned}
$$

Setting $T=\gamma(\omega)$ and applying $\gamma^{-1}$ to both sides give

$$
\begin{aligned}
& \mathbf{D}_{m}\left(\tau_{\mathscr{B}}(\omega)\right)=\tau_{\mathscr{B}}\left(\mathbf{D}_{m}(\omega)\right), \\
& \forall \omega \in \Omega_{\mathbb{K}}^{\gamma}(M) \Longleftrightarrow \mathbf{D}_{m} \circ \tau_{\mathscr{B}}=\tau_{\mathscr{B}} \circ \mathbf{D}_{m} .
\end{aligned}
$$

In particular, the $\mathbb{K}$-algebra of generalized Killing forms is invariant under $\tau_{\mathscr{B}}$ :

$$
\tau_{\mathscr{B}}\left(\check{\mathscr{K}}_{D}\right)=\check{\mathscr{K}}_{D},
$$

a property (by virtue of (206)) it shares with the flat Fierz $\mathbb{K}$ algebra $\check{\mathscr{K}}(D)$

$$
\tau_{\mathscr{B}}(\check{\mathscr{K}}(D))=\check{\mathscr{K}}(D) .
$$

Together with (205), identity (255) implies that D-flatness of $\check{E}_{\xi, \xi^{\prime}}$ and D-flatness of $\check{E}_{\xi^{\prime}, \xi}$ are equivalent statements, so that it suffices to require only one of the two.

5.8. Alternate Form of the Differential Constraints. Consider the following local expansion, which results by applying (209) to $\left[T, E_{\xi, \xi^{\prime}}\right]_{-, o}$, where $T \in \Gamma(M, \operatorname{End}(S))$ :

$$
\begin{aligned}
& {\left[T, E_{\xi, \xi^{\prime}}\right]_{-, o}} \\
& =\frac{1}{2^{[d / 2]}} \sum_{k=0}^{d} \frac{1}{k !} \operatorname{tr}\left(\gamma^{a_{k} \cdots a_{1}} \circ\left[T, E_{\xi, \xi^{\prime}}\right]_{-, o}\right) \gamma_{a_{1} \cdots a_{k}}, \\
& \forall T \in \Gamma(M, \operatorname{End}(S)), \forall \xi, \xi^{\prime} \in \Gamma(M, S) .
\end{aligned}
$$

An easy computation using cyclicity of tr and identity (208) gives

$$
\operatorname{tr}\left(\gamma^{a_{k} \cdots a_{1}} \circ\left[T, E_{\xi, \xi^{\prime}}\right]_{-, o}\right)=-\mathscr{B}\left(\left[T, \gamma^{a_{k} \cdots a_{1}}\right]_{-, o} \xi, \xi^{\prime}\right),
$$

so that (258) becomes

$$
\begin{aligned}
& {\left[T, E_{\xi, \xi^{\prime}}\right]_{-, \circ}} \\
& =-\frac{1}{2^{[d / 2]}} \sum_{k=0}^{d} \frac{1}{k !} \mathscr{B}\left(\left[T, \gamma^{a_{k} \cdots a_{1}}\right]_{-, \circ} \xi, \xi^{\prime}\right) \gamma_{a_{1} \cdots a_{k}}, \\
& \forall T \in \Gamma(M, \operatorname{End}(S)), \quad \forall \xi, \xi^{\prime} \in \Gamma(M, S) .
\end{aligned}
$$

Setting $T=A_{m}=\gamma\left(\check{A}_{m}\right)$ in (260) and applying the morphism $\gamma^{-1}$ to both sides give

$$
\begin{aligned}
& {\left[\check{A}_{m}, \check{\mathbf{E}}_{\xi, \xi^{\prime}}\right]_{-, \diamond}} \\
& \quad=-\sum_{k=0}^{d} \frac{1}{k !} \mathscr{B}\left(\left[A_{m}, \gamma^{a_{k} \cdots a_{1}}\right]_{-, o} \xi, \xi^{\prime}\right) e_{\gamma}^{a_{1} \cdots a_{k}} .
\end{aligned}
$$

Using this identity in the definition (236) of $\mathbf{D}_{m}$ gives

$$
\begin{aligned}
\mathbf{D}_{m} \check{\mathbf{E}}_{\xi, \xi^{\prime}}= & \nabla_{m} \check{\mathbf{E}}_{\xi, \xi^{\prime}} \\
& -\sum_{k=0}^{d} \frac{1}{k !} \mathscr{R}\left(\xi,\left[A_{m}, \gamma_{a_{1} \cdots a_{k}}\right]_{-, o} \xi^{\prime}\right) e_{\gamma}^{a_{1} \cdots a_{k}} .
\end{aligned}
$$

Consider now relation (246), written in terms of $\check{\mathbf{E}}_{\xi, \xi^{\prime}}$ :

$$
\mathbf{D}_{m} \check{\mathbf{E}}_{\bar{\xi}, \xi^{\prime}}=\check{\mathbf{E}}_{D_{m} \xi, \xi^{\prime}}+\check{\mathbf{E}}_{\xi, D_{m} \xi^{\prime}}, \quad \forall \xi, \xi^{\prime} \in \Gamma(M, S) .
$$

Substituting (262) in the left hand side, this becomes

$$
\begin{aligned}
\nabla_{m} \check{\mathbf{E}}_{\xi, \xi^{\prime}}= & \check{\mathbf{E}}_{D_{m} \xi, \xi^{\prime}}+\check{\mathbf{E}}_{\xi, D_{m} \xi^{\prime}} \\
& +\sum_{k=0}^{d} \frac{1}{k !} \mathscr{B}\left(\xi,\left[A_{m}, \gamma_{a_{1} \cdots a_{k}}\right]_{-, o} \xi^{\prime}\right) e_{\gamma}^{a_{1} \cdots a_{k}} .
\end{aligned}
$$

Separating ranks, we conclude that (246) is equivalent to the following system of identities:

$$
\begin{aligned}
& \nabla_{m} \check{\mathbf{E}}_{a_{1} \cdots a_{k}}\left(\xi, \xi^{\prime}\right)= \check{\mathbf{E}}_{a_{1} \cdots a_{k}}\left(D_{m} \xi, \xi^{\prime}\right) \\
&+\check{\mathbf{E}}_{a_{1} \cdots a_{k}}\left(\xi, D_{m} \xi^{\prime}\right) \\
&+\mathscr{B}\left(\xi,\left[A_{m}, \gamma_{a_{1} \cdots a_{k}}\right]_{-, o} \xi^{\prime}\right), \\
& \forall k=0 \cdots d,
\end{aligned}
$$

where $\xi, \xi^{\prime} \in \Gamma(M, S)$ are arbitrary. In particular, we have

$$
\begin{aligned}
\nabla_{m} \check{\mathbf{E}}_{a_{1} \cdots a_{k}}\left(\xi, \xi^{\prime}\right)=\mathscr{B}\left(\xi,\left[A_{m}, \gamma_{a_{1} \cdots a_{k}}\right]_{-, o} \xi^{\prime}\right), \\
\forall k=0 \cdots d, \forall \xi, \xi^{\prime} \in \mathscr{K}(D),
\end{aligned}
$$

which agrees with (B.12) (see Appendix B.2).

5.9. The $\mathbb{K}$-Algebra of Constrained Generalized Killing Forms. As before, we consider the case $\chi=1$ of the CGK equations:

$$
D \xi=Q \xi=0, \quad \text { with } D=\nabla^{S}+A, A=\mathrm{d} x^{m} \otimes A_{m} .
$$

Let $\mathscr{K}(D, Q)=\mathscr{K}(D) \cap \mathscr{K}(Q)$ denote the (finitedimensional) $\mathbb{K}$-linear subspace of $\Gamma(M, S)$ consisting of all solutions to (267). We define the $\mathbb{K}$-algebra of constrained generalized Killing (CGK) forms determined by $D$ and $Q$ to be the following $\mathbb{K}$-subalgebra of $\left(\Omega_{\mathbb{K}}^{\gamma}(M), \diamond\right)$ :

$$
\check{\mathscr{K}}_{D, \mathrm{Q}} \stackrel{\text { def }}{=} \check{\mathscr{K}}_{D} \cap \check{\mathscr{K}}_{\mathrm{Q}} \text {. }
$$

In general, $\check{\mathscr{K}}_{D, Q}$ is a nonunital $\mathbb{K}$-algebra. The discussion of the previous subsections shows that the flat Fierz $\mathbb{K}$-algebra determined by the $\mathscr{B}$-flat subspace $\mathscr{K}(D, Q) \subset \Gamma(M, S)$

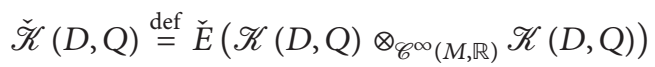

is a subalgebra of the $\mathbb{K}$-algebra of CGK forms:

$$
\check{\mathscr{K}}(D, Q) \subset \check{\mathscr{K}}_{D, Q} .
$$


This property of the Fierz isomorphism depends essentially on the assumption that $\mathscr{B}$ is $D$-flat (an assumption which is satisfied in the application discussed in Section 6).

Expression for a Basis of Solutions of the CGK Pinor Equations. Let $s=\operatorname{dim}_{\mathbb{K}} \mathscr{K}(D, Q)$ denote the $\mathbb{K}$-dimension of the space of solutions to the CGK equations. Choosing a basis $\left(\xi_{i}\right)_{i=1 \cdots s}$ of such solutions, we set

$$
\check{E}_{i j} \stackrel{\text { def }}{=} \check{E}_{\xi_{i}, \xi_{j}}=\frac{1}{2^{[(d+1) / 2]}} \check{\mathbf{E}}_{i j} \in \Omega_{\mathbb{K}}^{\gamma}(M),
$$

where (cf. equation (218))

$$
\check{\mathbf{E}}_{i j}=\sum_{k=0}^{d} \check{\mathbf{E}}_{i j}^{(k)},
$$

with

$$
\begin{aligned}
\check{\mathbf{E}}_{i j}^{(k)} \stackrel{\text { def }}{=} \check{\mathbf{E}}_{\xi_{i}, \xi_{j}}^{(k)} & =\frac{1}{\mathrm{k} !} \check{\mathbf{E}}_{a_{1} \cdots a_{k}}^{(k)}\left(\xi_{i}, \xi_{j}\right) e_{\gamma}^{a_{1} \cdots a_{k}}, \\
\check{\mathbf{E}}_{a_{1} \cdots a_{k}}^{(k)}\left(\xi_{i}, \xi_{j}\right) & =\mathscr{B}\left(\gamma_{a_{k} \cdots a_{1}} \xi_{i}, \xi_{j}\right) \\
& =\epsilon_{\mathscr{B}}^{k} \mathscr{B}\left(\xi_{i}, \gamma_{a_{1} \cdots a_{k}} \xi_{j}\right)
\end{aligned}
$$

giving the following expression for the homogeneous formvalued bilinears:

$$
\check{\mathbf{E}}_{i j}^{(k)}={ }_{U} \frac{1}{k !} \epsilon_{\mathscr{B}}^{k} \mathscr{B}\left(\xi_{i}, \gamma_{a_{1} \cdots a_{k}} \xi_{j}\right) e_{\gamma}^{a_{1} \cdots a_{k}}
$$

Since $\xi_{i} \otimes \xi_{j}$ form a basis of the $\mathbb{K}$-vector space $\mathscr{K}(D, Q) \otimes_{\mathscr{C}{ }^{\infty}(M, \mathbb{R})} \mathscr{K}(D, Q)$, the inhomogeneous differential forms $\check{E}_{i j}$ form a basis of the $\mathbb{K}$-vector space $\check{\mathscr{K}}(D, Q)$. Inclusion (270) amounts to the following system of equations for the inhomogeneous differential forms $\check{E}_{i j}$ :

$$
\mathbf{D}_{m} \check{E}_{i j}=\check{Q} \diamond \check{E}_{i j}=\check{E}_{i j} \diamond \tau_{\mathscr{B}}(\check{Q})=0 \text {, }
$$

$$
\forall i, j=1 \cdots s
$$

These can also be written as follows:

$$
\mathbf{D}_{m} \check{E}_{i j}=\check{Q} \diamond \check{E}_{i j}=0, \quad \forall i, j=1 \cdots s
$$

upon applying $\tau_{\mathscr{B}}$ to the last equation in (275) and using the relation $\tau_{\mathscr{B}}\left(\check{E}_{i j}\right)=\epsilon_{\mathscr{B}} \check{E}_{j i}$ (cf. (205)). The inhomogeneous differential forms $\check{E}_{i j}$ also satisfy the Fierz identities:

$$
\check{E}_{i j} \diamond \check{E}_{k l}=\mathscr{B}_{k j} \check{E}_{i l}, \quad \forall i, j, k, l=1 \cdots s,
$$

where we defined the following constants:

$$
\mathscr{B}_{i j} \stackrel{\text { def }}{=} \mathscr{B}\left(\xi_{i}, \xi_{j}\right) \text {. }
$$

The algebrodifferential system consisting of (276) and (277) can be taken as the basis for extending the classical theory of Killing forms, a subject which is of mathematical interest in its own right. It provides a synthetic geometric description of the essential conditions imposed by having a fixed number of unbroken supersymmetries in a flux compactification, formulated in the language of geometric algebra. When expanding the geometric product into generalized products using (35), the innocently looking equations (276) and (277) take on a form which may seem rather formidable when $s$ is sufficiently large (see Section 6 for an example). Of course, the language of geometric algebra allows one to study such systems starting directly from the synthetic expressions (276) and (277), which shows that the problem of characterizing the solutions of such equations belongs most properly to the intersection between Kähler-Cartan theory [17] and the theory of noncommutative associative algebras-a point of view on flux compactifications, which, in our opinion, could lead to a deeper understanding of various problems pertaining to that subject.

Truncated Model of the $\mathbb{K}$-Algebra of CGK Forms in the Nonsimple Case. Recall that, in the nonsimple case, we can realize $\left(\Omega^{\epsilon}(M), \diamond\right)$ as the truncated algebra $\left(\Omega^{<}(M), \diamond_{\epsilon}\right)$, where $\epsilon \in\{-1,+1\}$ is the signature of $\gamma$. An inhomogeneous form $\omega \in \Omega^{\epsilon}(M)$ satisfies $\widetilde{*} \omega=\epsilon \omega$ and is called twisted self-dual if $\epsilon=+1$ and twisted anti-self-dual if $\epsilon=-1$. Such forms can be uniquely decomposed as $\omega=2 P_{\epsilon}\left(\omega_{<}\right)=$ $\omega_{<}+\epsilon \tilde{*} \omega_{<}$, where $\omega_{<} \in \Omega^{<}(M)$ has rank smaller than $[d / 2]$. In particular, the forms $\check{E}_{i j}$ discussed above can be decomposed uniquely as $\check{E}_{i j}=\check{E}_{i j}^{<}+\epsilon \widetilde{*} \check{E}_{i j}^{<}$. Similarly, we have the unique decompositions $\check{Q}=\check{Q}^{<}+\epsilon \widetilde{*} \check{Q}^{<}$and $\check{A}_{m}=\check{A}_{m}^{<}+\epsilon \widetilde{*} \check{A}_{m}^{<}$, where $\epsilon=\epsilon_{\gamma}$. Let us define a derivation $\check{D}_{m}^{\text {ad, }<}$ of $\left(\Omega(M), \diamond_{\epsilon}\right)$ through

$$
\begin{gathered}
\check{D}_{m}^{\mathrm{ad},<}(\omega) \stackrel{\text { def }}{=} \nabla_{m} \omega+2\left[\check{A}_{m}^{<}, \omega\right]_{-, \bullet_{\epsilon}} \\
\quad=\nabla_{m} \omega+\left(\left[\check{A}_{m}, \omega\right]_{-, \diamond}\right)^{<}=\left(\mathbf{D}_{m} \omega\right)^{<},
\end{gathered}
$$

$\forall \omega \in \Omega^{<}(M)$,

where, as usual, the (anti-)commutator with respect to $\diamond_{\epsilon}$ is defined as follows:

$$
[\omega, \eta]_{ \pm, \bullet_{\epsilon}}=\omega \diamond_{\epsilon} \eta \pm \eta \diamond_{\epsilon} \omega, \quad \forall \omega, \eta \in \Omega^{<}(M)
$$

We have

$$
\begin{aligned}
\left([\omega, \eta]_{ \pm, \diamond}\right)^{<}=P_{<}\left([\omega, \eta]_{ \pm, \diamond}\right)=2\left[\omega_{<}, \eta_{<}\right]_{-, \bullet_{e}} & \\
& \forall \omega, \eta \in \Omega(M)
\end{aligned}
$$

since $2 P_{<}$is a morphism of algebras from $(\Omega(M), \diamond)$ to $\left(\Omega(M), \diamond_{\epsilon}\right)$. Noticing that $\left[\nabla_{m}, P_{<}\right]_{-, \circ}=0$, we find that $\check{D}_{m}^{\text {ad, }<}$ satisfies

$$
P_{<} \circ \mathbf{D}_{m}=\check{D}_{m}^{\mathrm{ad},<} \circ P_{<}
$$

which generalizes (279).

Using the commutation relation $\left[\nabla_{m}, P_{\epsilon}\right]_{-, o}=0$ and the mutually inverse isomorphisms of diagram (107), it is easy to 
see that the CGK pinor equations (276) are equivalent to the truncated CGK pinor equations:

$$
\check{D}_{m}^{\mathrm{ad},<} \check{E}_{i j}^{<}=\check{Q}^{<} \check{\iota}_{\epsilon} \check{E}_{i j}^{<}=0, \quad \forall i, j=1 \cdots s .
$$

Indeed, applying $2 P_{<}$to (276) gives (283) while applying $P_{\epsilon}$ to (283) gives (276). The first of the truncated CGK pinor equations can also be written in the form $\nabla_{m} \check{E}_{i j}^{<}=$ $-2\left[\check{A}_{m}, \check{E}_{i j}^{<}\right]_{-, \bullet_{e}}$. On the other hand, applying $2 P_{<}$to $(277)$ gives the truncated geometric Fierz identities:

$$
\check{E}_{i j}^{<} \breve{E}_{\epsilon l}^{<}=\frac{1}{2} \mathscr{B}_{k j} \check{E}_{i l}^{<}, \quad \forall i, j, k, l=1 \cdots s .
$$

5.10. A Particular Case: When $\mathbb{K}=\mathbb{R}$ and $\mathscr{B}$ Is a Scalar Product. Consider the particular case when $\mathbb{K}=\mathbb{R}$ and $\mathscr{B}$ is symmetric (thus $\sigma_{\mathscr{B}}=+1$ ) and positive-definite with $\epsilon_{\mathscr{B}}=+1$ (this happens, e.g., in the application considered in Section 6). In this case, we can choose $\xi_{1}, \ldots, \xi_{s}$ such that $\mathscr{B}_{i j}=\delta_{i j}$. Then relations (277) show that the $\mathbb{K}$-algebra $\check{\mathscr{K}}(D, Q)$ is isomorphic with the algebra $\operatorname{Mat}(s, \mathbb{R})$ of square real matrices of dimension $s$, the unit being given by $\check{C} \stackrel{\text { def }}{=}$ $\sum_{i=1}^{s} \check{E}_{i i}$. An isomorphism to $\operatorname{Mat}(s, \mathbb{R})$ is given by $\check{E}_{i j} \rightarrow e_{i j}$, where $e_{i j} \in \operatorname{Mat}(s, \mathbb{R})$ is the matrix whose only nonvanishing entry equals 1 and is found on the $i$ th row and $j$ th column:

$$
\left(e_{i j}\right)_{k l}=\delta_{i k} \delta_{j l}
$$

We have $\check{C}^{\diamond 2}=\check{C}, \check{C} \diamond \check{E}_{i j}=\check{E}_{i j} \diamond \check{C}=\check{E}_{i j}, \check{E}_{i i}^{\diamond 2}=\check{E}_{i i}, \check{E}_{i j}^{\diamond 2}=0$ for $i \neq j$. Since $\check{E}_{i j} \diamond \check{E}_{j i}=\check{E}_{i i}$, the $\mathbb{K}$-algebra $\check{\mathscr{K}}(D, Q)$ is generated by the elements $\left(\check{E}_{i j}\right)_{i \neq j}$, with the relations:

$$
\begin{aligned}
\check{E}_{i j} \diamond \check{E}_{k l} & =0, \quad \forall i \neq j, \quad j \neq k, k \neq l, \\
\check{E}_{i j} \diamond \check{E}_{j k} & =\check{E}_{i k}, \quad \forall i, j, k \text { distinct, } \\
\check{E}_{i j} \diamond \check{E}_{j i} \diamond \check{E}_{i k} & =\check{E}_{i k}, \quad \forall i \neq j, \quad i \neq k, \\
\check{E}_{i j} \diamond \check{E}_{j k} \diamond \check{E}_{k j} & =\check{E}_{i j}, \quad \forall i \neq j, \quad j \neq k .
\end{aligned}
$$

This system of generators and relations can be reduced further (see the example below) upon using the identity $\check{E}_{j i}=$ $\tau\left(\check{E}_{i j}\right)$, where we used the fact that $\sigma_{\mathscr{B}}=+1$ and we noticed that $\tau_{\mathscr{B}}=\tau\left(\right.$ since $\left.\epsilon_{\mathscr{B}}=+1\right)$.

The Case of One CGK Pinor. When $s=1$ (a single unit norm solution $\xi$ of the CGK pinor equations, which is unique up to sign), the flat Fierz $\mathbb{K}$-algebra $\check{\mathscr{K}}(D, Q)$ has dimension one, being isomorphic with $\mathbb{R}$ as a unital $\mathbb{R}$-algebra. It is generated by the single basis element $\check{E}=\check{E}_{\xi, \xi}$ (which is the unit of $\check{\mathscr{K}}(D, Q))$, with the relation

$$
\check{E} \diamond \check{E}=\check{E} .
$$

The condition $\tau(\check{E})=\check{E}$ implies that certain rank components of $\check{E}$ vanish identically. This situation occurs in the example considered in Section 6, though in that case we prefer to take the squared norm $\mathscr{B}(\xi, \xi)$ of $\xi$ to equal 2 , for ease of comparison with the results of [3]. This produces an extra factor of two in the right hand side of (287).

The Case of Two CGK Pinors. When $s=2$, the flat Fierz $\mathbb{K}$-algebra $\check{\mathscr{K}}(D, Q)$ is generated by $\check{E}_{12}$ and $\check{E}_{21}$ with the relations

$$
\check{E}_{12}^{\diamond 2}=\check{E}_{21}^{\diamond 2}=0
$$

$$
\check{E}_{12} \diamond \check{E}_{21} \diamond \check{E}_{12}-\check{E}_{12}=\check{E}_{21} \diamond \check{E}_{12} \diamond \check{E}_{21}-\check{E}_{21}=0 \text {. }
$$

We have $\check{E}_{11}=\check{E}_{12} \diamond \check{E}_{21}, \check{E}_{22}=\check{E}_{21} \diamond \check{E}_{12}$ and $\check{C}=\check{E}_{11}+$ $\check{E}_{22}=\check{E}_{12} \diamond \check{E}_{21}+\check{E}_{21} \diamond \check{E}_{12}$. Since $\check{E}_{21}=\tau\left(\check{E}_{12}\right)$, we can in fact generate the entire algebra from $\check{E}_{12}$ up to applying $\tau$. Using the fact that the first and second equation in (288) as well as the first and second equation in (289) are related through reversion, we find that the entire system is equivalent to the following Fierz relations:

$$
\begin{gathered}
\check{E}_{12}^{\diamond 2}=0, \\
\check{E}_{12} \diamond \tau\left(\check{E}_{12}\right) \diamond \check{E}_{12}=\check{E}_{12},
\end{gathered}
$$

together with the following $Q$ - and $D$-constraints:

$$
\begin{aligned}
\check{Q} \diamond \check{E}_{12} & =\check{E}_{12} \diamond \tau(\check{Q})=0, \\
\mathbf{D}_{m} \check{E}_{12} & =0 .
\end{aligned}
$$

Remark 12. The algebra $\operatorname{Mat}(2, \mathbb{R}) \approx \mathrm{Cl}(2,0)$ is also generated by two real Pauli matrices, for example, by

$$
\begin{aligned}
& \sigma_{1}=\left[\begin{array}{ll}
0 & 1 \\
1 & 0
\end{array}\right]=e_{12}+e_{21}, \\
& \sigma_{3}=\left[\begin{array}{cc}
1 & 0 \\
0 & -1
\end{array}\right]=e_{11}-e_{22}
\end{aligned}
$$

subject to the relations $\sigma_{1}^{2}=\sigma_{3}^{2}=1$ and $\sigma_{1} \sigma_{3}+\sigma_{3} \sigma_{1}=0$. Therefore, the flat Fierz $\mathbb{K}$-algebra $\check{\mathscr{K}}(D, Q)$ is also generated by the inhomogeneous differential forms:

$$
\begin{aligned}
& \check{\Sigma}_{1}=\check{E}_{12}+\check{E}_{21}, \\
& \check{\Sigma}_{3}=\check{E}_{11}-\check{E}_{22},
\end{aligned}
$$

subject to the relations

$$
\begin{aligned}
\check{\Sigma}_{1}^{\diamond 2}-\check{\Sigma}_{3}^{\diamond 2}=0, \\
\check{\Sigma}_{1} \diamond \check{\Sigma}_{3}+\check{\Sigma}_{3} \diamond \check{\Sigma}_{1}=0, \\
\check{\Sigma}_{1}^{\diamond 3}=\check{\Sigma}_{1}, \\
\check{\Sigma}_{3}^{\diamond 3}=\check{\Sigma}_{3} .
\end{aligned}
$$


Noticing that $\check{\Sigma}_{1} \diamond \check{\Sigma}_{3}=\check{E}_{21}-\check{E}_{12}$, we have $\check{C}=\check{E}_{11}+\check{E}_{22}=$ $\check{\Sigma}_{1}^{\diamond 2}=\check{\Sigma}_{3}^{\diamond 2}$ and

$$
\begin{aligned}
& \check{E}_{11}=\frac{1}{2}\left(\check{C}+\check{\Sigma}_{3}\right), \\
& \check{E}_{22}=\frac{1}{2}\left(\check{C}-\check{\Sigma}_{3}\right), \\
& \check{E}_{12}=\frac{1}{2}\left(\check{\Sigma}_{1}-\check{\Sigma}_{1} \diamond \check{\Sigma}_{3}\right), \\
& \check{E}_{21}=\frac{1}{2}\left(\check{\Sigma}_{1}+\check{\Sigma}_{1} \diamond \check{\Sigma}_{3}\right) .
\end{aligned}
$$

\section{Example: Flux Compactifications of Eleven-Dimensional Supergravity on Eight Manifolds}

In this section, we illustrate the methods developed in the present paper by applying them to the case of $\mathcal{N}=1$ warped compactifications of eleven-dimensional supergravity on eight manifolds down to an $\mathrm{AdS}_{3}$ space, a situation which was studied through direct methods in [3,4]. After some basic preparations in Section 6.1, Section 6.2 gives our translation of the generalized Killing pinor equations into a system of algebraic and differential constraints on inhomogeneous forms defined on the compactification space and shows how our approach allows one to recover the results of [3].

6.1. Preparations. Consider supergravity on an 11-manifold $\widetilde{M}$ with Lorentzian metric $\widetilde{g}$ (of "mostly plus" signature). Besides the metric, the action of the theory contains the three-form potential with four-form field strength $\widetilde{G} \in$ $\Omega^{4}(\widetilde{M})$ and the gravitino $\widetilde{\Psi}_{M}$, which is a real pinor of spin $3 / 2$. We assume that $(\widetilde{M}, \widetilde{g})$ is spinnable. For supersymmetric bosonic backgrounds, both the gravitino VEV and its supersymmetry variation must vanish, which requires that there exists at least one solution $\tilde{\eta}$ to the equation:

$$
\delta_{\widetilde{\eta}} \widetilde{\Psi}_{M} \stackrel{\text { def }}{=} \widetilde{\mathscr{D}}_{M} \widetilde{\eta}=0
$$

where uppercase indices run from 0 to 10 and $\widetilde{\mathscr{D}}_{M}$ is the supercovariant connection:

$$
\begin{aligned}
\widetilde{\mathscr{D}}_{M} & \stackrel{\text { def }}{=} \nabla_{M}^{\widetilde{S}} \\
& -\frac{1}{288}\left(\widetilde{G}_{N P Q R} \widetilde{\gamma}_{M}^{N P Q R}-8 \widetilde{G}_{M N P Q} \widetilde{\gamma}^{N P Q}\right) .
\end{aligned}
$$

Here, $\tilde{\gamma}^{M}$ are the gamma matrices of $\mathrm{Cl}(10,1)$ in that 32 dimensional real (Majorana) irreducible representation for which $\tilde{\gamma}^{(12)} \stackrel{\text { def }}{=} \tilde{\gamma}^{1} \cdots \tilde{\gamma}^{11}=+1$ and

$$
\nabla_{M}^{\widetilde{S}}=\partial_{M}+\frac{1}{4} \widetilde{\Omega}_{M N P} \widetilde{\gamma}^{N P}
$$

is the connection on the pin bundle $\widetilde{S}$ induced by the Levi-Civita connection of $(\widetilde{M}, \widetilde{g})$. The eleven-dimensional supersymmetry generator $\tilde{\eta}$ (which is a Majorana spinor field of spin $1 / 2$ ) is a smooth section of the pin bundle $\widetilde{S}$, which is a rank 32 real vector bundle defined on $\widetilde{M}$.

As in [3], we consider compactification down to an $\mathrm{AdS}_{3}$ space of cosmological constant $\Lambda=-8 \kappa^{2}$, where $\kappa$ is a positive real parameter; this includes the Minkowski case as the limit $\kappa \rightarrow 0$. Thus $\widetilde{M}=N \times M$, where $N$ is an oriented 3-manifold diffeomorphic with $\mathbb{R}^{3}$ and carrying the $\mathrm{AdS}_{3}$ metric while $M$ is an oriented Riemannian eight-manifold whose metric we denote by $g$. The metric on $\widetilde{M}$ is a warped product:

$$
\mathrm{d} \widetilde{s}_{11}^{2}=e^{2 \Delta} \mathrm{d} s_{11}^{2}, \quad \text { where } \mathrm{d} s_{11}^{2}=\mathrm{d} s_{3}^{2}+g_{m n} \mathrm{~d} x^{m} \mathrm{~d} x^{n} .
$$

Here, the warp factor $\Delta$ is a smooth function defined on $M$ while $\mathrm{d} s_{3}^{2}$ is the squared length element on $N$. For the field strength $\widetilde{G}$, we use the ansatz:

$$
\widetilde{G}=e^{3 \Delta} G \quad \text { with } G=\operatorname{vol}_{3} \wedge f+F,
$$

where $f=f_{m} e^{m} \in \Omega^{1}(M), F=(1 / 4 !) F_{m n p q} e^{m n p q} \in \Omega^{4}(M)$, and $\mathrm{vol}_{3}$ is the volume form of $N$. Small Latin indices from the middle of the alphabet run from 1 to 8 and correspond to a choice of frame on $M$. For $\tilde{\eta}$, we use the ansatz:

$$
\widetilde{\eta}=e^{\Delta / 2} \eta \quad \text { with } \eta=\psi \otimes \xi
$$

where $\xi$ is a Majorana spinor of spin $1 / 2$ (a.k.a. a real pinor) on the internal space $M$ and $\psi$ is a Majorana spinor on the $\mathrm{AdS}_{3}$ space $N$. Mathematically, $\xi$ is a section of the pinor bundle of $M$, which is a real vector bundle of rank 16 defined on $M$, carrying a fiberwise representation of the Clifford algebra $\mathrm{Cl}(8,0)$. Since $p-q \equiv_{8} 0$ for $p=8$ and $q=0$, this corresponds to the simple normal case of Section 4. In particular, the corresponding morphism $\gamma:\left(\wedge T^{*} M, \diamond\right) \rightarrow(\operatorname{End}(S), \circ)$ of bundles of algebras is an isomorphism; that is, it is bijective on the fibers. We set $\gamma^{m}=\gamma\left(e^{m}\right)$ for some local frame of $M$. In dimension eight with Euclidean signature, there exists an admissible [30] (and thus Spin(8)-invariant) bilinear pairing $\mathscr{B}$ on the pin bundle $S$ with $\sigma_{\mathscr{B}}=+1$ and $\epsilon_{\mathscr{B}}=+1$ which is a scalar product (i.e., it is fiberwise symmetric and positivedefinite).

Assuming that $\psi$ is a Killing pinor on the $\mathrm{AdS}_{3}$ space, the supersymmetry condition (296) decomposes into a system consisting of the following constraints for $\xi$ :

$$
\begin{aligned}
D_{m} \xi & =0, \\
Q \xi & =0,
\end{aligned}
$$

where $D_{m}$ is a linear connection on $S$ and $Q \in \Gamma(M, \operatorname{End}(S))$ is a globally defined endomorphism of the vector bundle $S$. As in $[3,4]$, we do not require that $\xi$ has definite chirality (i.e., $\xi$ need not satisfy the Weyl condition). The space of solutions of (302) is a finite-dimensional $\mathbb{R}$-linear subspace $\mathscr{K}(D, Q)$ of the space $\Gamma(M, S)$ of smooth sections of $S$. Of course, this subspace is trivial for generic metrics $g$ and fluxes $F$ and $f$ on $M$, since the generic compactification of the type we consider breaks all supersymmetry. The interesting problem is to find 
those metrics and fluxes on $M$ for which some fixed amount of supersymmetry is preserved in three dimensions, that is, for which the space $\mathscr{K}(D, Q)$ has some given nonvanishing dimension, which we denote by $s$. The case $s=1$ (which corresponds to $\mathcal{N}=1$ supersymmetry in three dimensions) was studied in [3,4] and will be reconsidered below. Direct computation using the compactification ansatz gives the following expressions for $D$ and $Q$ (which are equivalent to those derived in reference [3]; see the remark below for ease of comparison):

$$
\begin{aligned}
D_{m}= & \nabla_{m}^{S}+A_{m}, \\
A_{m} & =\frac{1}{4} f_{p} \gamma_{m}^{p} \circ \gamma^{(9)}+\frac{1}{24} F_{m p q r} \gamma^{p q r}+\kappa \gamma_{m} \circ \gamma^{(9)}, \\
Q & =\frac{1}{2} \gamma^{m} \partial_{m} \Delta-\frac{1}{288} F_{m p q r} \gamma^{m p q r}-\frac{1}{6} f_{p} \gamma^{p} \circ \gamma^{(9)} \\
& -\kappa \gamma^{(9)},
\end{aligned}
$$

where $\gamma^{(9)}=\gamma^{1} \circ \ldots \circ \gamma^{8}$. Notice that the last terms in (303) and (304) depend on the cosmological constant of the $\mathrm{AdS}_{3}$ space and that they vanish in the Minkowski limit $\kappa \rightarrow 0$.

Given some desired amount of supersymmetry which we want to be preserved in three dimensions (i.e., given some desired dimensionality $s$ of the space of solutions to (302)), the general aim is to reformulate (302) as equations on differential forms $\check{\mathbf{E}}_{\xi, \xi^{\prime}}^{(k)}=(1 / k !) \mathscr{B}\left(\xi, \gamma_{m_{1} \cdots m_{k}} \xi^{\prime}\right) e^{a_{1} \cdots a_{m}}$ constructed as bilinear combinations of pinors $\xi, \xi^{\prime}$ which satisfy (302). The pinor bilinears will be constrained by Fierz identities. The translation to equations on the differential forms $\check{\mathbf{E}}_{\xi, \xi^{\prime}}^{(k)}$ can be achieved directly by starting from the following equivalent reformulation of the algebraic constraints $Q \xi=Q \xi^{\prime}=0$

$$
\mathscr{B}\left(\xi,\left(Q^{t} \circ \gamma_{m_{1} \cdots m_{k}} \pm \gamma_{m_{1} \cdots m_{k}} \circ Q\right) \xi^{\prime}\right)=0
$$

and treating the constraints $D_{m} \xi=D_{m} \xi^{\prime}=0$ through the method outlined in [3]. The theoretical basis of that approach is explained in detail in Appendix B, where we also show how that method is equivalent to the more general approach which we have developed in the present paper. In the next subsection, we will illustrate our approach in the simplest case $s=1(\mathcal{N}=1$ supersymmetry in three dimensions, see [3] $)$, so we will require that (302) admits one nontrivial solution $\xi$.

Remark 13. For easy comparison with [3], we note that loc. cit. uses a redundant parameterization of the degrees of freedom described by $\xi$, which is given by the following sections of $S$ :

$$
\varepsilon^{ \pm} \stackrel{\text { def }}{=} \frac{1}{\sqrt{2}}\left(\xi_{+} \pm \xi_{-}\right)
$$

which satisfy $\gamma^{(9)} \varepsilon^{ \pm}=\varepsilon^{\mp}$. Here, $\xi_{ \pm}=\mathscr{P}_{ \pm} \xi$ are the positive and negative chirality components of $\xi$, with $\mathscr{P}_{ \pm} \stackrel{\text { def }}{=}(1 / 2)\left(1 \pm \gamma^{(9)}\right)$.
We have $\xi=\xi_{+}+\xi_{-}$and $\gamma^{(9)} \xi=\xi_{+}-\xi_{-}$with $\gamma^{(9)} \xi_{ \pm}= \pm \xi_{ \pm}$. The operators $\mathscr{P}_{ \pm}$are complementary $\mathscr{B}$-orthoprojectors:

$$
\begin{aligned}
\mathscr{P}_{ \pm}^{2} & =\mathscr{P}_{ \pm}, \\
\mathscr{P}_{ \pm} \mathscr{P}_{\mp} & =0, \\
\mathscr{P}_{+}+\mathscr{P}_{-} & =1, \\
\left(\mathscr{P}_{ \pm}\right)^{t} & =\mathscr{P}_{ \pm} .
\end{aligned}
$$

In particular, one has

$$
\begin{aligned}
& \varepsilon^{+}=\frac{1}{\sqrt{2}} \xi, \\
& \varepsilon^{-}=\frac{1}{\sqrt{2}} \gamma^{(9)} \xi,
\end{aligned}
$$

so $\varepsilon^{+}$and $\varepsilon^{-}=\gamma^{(9)} \varepsilon^{+}$are not independent. For reader's convenience, we note the identities:

$$
\begin{aligned}
& \mathscr{P}_{\epsilon_{1}} \gamma^{a_{1} \cdots a_{k}} \mathscr{P}_{\epsilon_{2}}=0 \Longrightarrow \mathscr{B}\left(\xi_{\epsilon_{1}}, \gamma^{a_{1} \cdots a_{k}} \xi_{\epsilon_{2}}\right)=0, \\
& \text { when } \epsilon_{1} \epsilon_{2}=(-1)^{k+1}, \\
& \mathscr{P}_{\epsilon_{1}} \gamma^{a_{1} \cdots a_{k}} \mathscr{P}_{\epsilon_{2}}=\mathscr{P}_{\epsilon_{1}} \gamma^{a_{1} \cdots a_{k}} \Longrightarrow \\
& \mathscr{B}\left(\xi_{\epsilon_{1}}, \gamma^{a_{1} \cdots a_{k}} \xi_{\epsilon_{2}}\right)=\mathscr{B}\left(\xi, \mathscr{P}_{\epsilon_{1}} \gamma^{a_{1} \cdots a_{k}} \xi\right), \\
& \text { when } \epsilon_{1} \epsilon_{2}=(-1)^{k},
\end{aligned}
$$

where $\epsilon_{1}, \epsilon_{2} \in\{-1,+1\}$ and $\mathscr{P}_{ \pm 1} \stackrel{\text { def }}{=} \mathscr{P}_{ \pm}, \xi_{ \pm 1} \stackrel{\text { def }}{=} \xi_{ \pm}$. Since $\epsilon_{\mathscr{B}}=+1$, (184) implies

$$
\left(\gamma^{a_{1} \cdots a_{k}}\right)^{t}=(-1)^{k(k-1) / 2} \gamma^{a_{1} \cdots a_{k}},
$$

which shows that $\gamma^{a_{1} \cdots a_{k}}$ is $\mathscr{B}$-symmetric for $k=0,1,4,5,8$ and $\mathscr{B}$-antisymmetric for $k=2,3,6,7$. In particular, we have that $\gamma^{(9)}$ is $\mathscr{B}$-symmetric.

6.2. The Case of $\mathcal{N}=1$ Supersymmetry in 3 Dimensions. Let us consider the CGK pinor equations (302) on the Riemannian 8-manifold $(M, g)$, assuming that the space of solutions has dimension $s=1$ over $\mathbb{R}$. Since $p-q \equiv_{8} 0$, we are in the normal simple case. This case is characterized by two admissible pairings $\mathscr{B}_{ \pm}$on $S$, which have the properties given in [31]; that is, $\epsilon_{\mathscr{B}_{+}}= \pm 1$ and $\sigma_{\mathscr{B}_{+}}=+1$. Since any choice of admissible pairing leads to the same result, we choose to work with $\mathscr{B}=\mathscr{B}_{+}$for convenience; this satisfies $\epsilon_{\mathscr{B}}=+1$ and $\sigma_{\mathscr{B}}=+1$. We can assume that $\mathscr{B}$ is a scalar product on $S$ and we denote the corresponding norm by \|\| .

As in Section 5.9 (see equation (271)), consider the inhomogeneous differential form:

$$
\check{E} \stackrel{\text { def }}{=} \frac{1}{16} \sum_{k=0}^{8} \check{\mathbf{E}}^{(k)} \in \Omega(M)
$$


defined by a nontrivial solution $\xi$ of (302). Equation (274) gives

$$
\begin{aligned}
& \check{\mathbf{E}}^{(k)}=\frac{1}{k !} \mathscr{B}\left(\xi, \gamma_{m_{1} \cdots m_{k}} \xi\right) e^{m_{1} \cdots m_{k}} \in \Omega^{k}(M), \\
& \forall a_{1}, \ldots, a_{k}=1 \cdots 8, \forall k=0 \cdots 8 .
\end{aligned}
$$

Using the properties of $\mathscr{B}$ and relations (178) and (184), one easily checks that $\check{\mathbf{E}}^{(2)}=\check{\mathbf{E}}^{(3)}=\check{\mathbf{E}}^{(6)}=\check{\mathbf{E}}^{(7)}=0$ while the nonvanishing bilinears are

$$
\begin{aligned}
\check{\mathbf{E}}^{(0)} & =\mathscr{B}(\xi, \xi), \\
\check{\mathbf{E}}^{(1)} & =\mathscr{B}\left(\xi, \gamma_{a} \xi\right) e^{a}, \\
\check{\mathbf{E}}^{(4)} & =\frac{1}{4 !} \mathscr{B}\left(\xi, \gamma_{a_{1} \cdots a_{4}} \xi\right) e^{a_{1} \cdots a_{4}}, \\
\check{\mathbf{E}}^{(5)} & =\frac{1}{5 !} \mathscr{B}\left(\xi, \gamma_{a_{1} \cdots a_{5}} \xi\right) e^{a_{1} \cdots a_{5}}, \\
\check{\mathbf{E}}^{(8)} & =\frac{1}{8 !} \mathscr{B}\left(\xi, \gamma_{a_{1} \cdots a_{3}} \xi\right) e^{a_{1} \cdots a_{8}}=\mathscr{B}\left(\xi, \gamma_{(9)} \xi\right) \nu,
\end{aligned}
$$

where $v$ is the volume form. Thus the inhomogeneous form which generates the Fierz algebra expands as

$$
\check{E}=\frac{1}{16}\left[\check{\mathbf{E}}^{(0)}+\check{\mathbf{E}}^{(1)}+\check{\mathbf{E}}^{(4)}+\check{\mathbf{E}}^{(5)}+\check{\mathbf{E}}^{(8)}\right] .
$$

Note that this case also admits nonvanishing 3- and 7-form bilinears, which are proportional to the Hodge duals of the 5- and 1-forms, respectively (but they do not appear in the generator). These other form-valued bilinears can also be expressed as in (274), but upon using the other admissible pairing $\mathscr{B}_{-}$, which is in fact not necessary for this analysis.

Remark 14. If one wanted to write the form-valued pinor bilinears in terms of the other admissible pairing $\mathscr{B}_{-}=\mathscr{B}_{+}$。 $\left(\mathrm{id}_{S} \otimes \gamma(\nu)\right)$, then one would be interested in constructing

$$
\begin{aligned}
\check{\mathbf{E}}_{-}^{(k)} & \stackrel{\operatorname{def}}{=} \frac{1}{k !}\left(\epsilon_{\mathscr{B}_{-}}\right)^{k} \mathscr{B}_{-}\left(\xi, \gamma_{a_{1} \cdots a_{k}} \xi\right) e^{a_{1} \cdots a_{k}} \\
& =\frac{1}{k !}(-1)^{k} \mathscr{B}\left(\xi, \gamma_{a_{1} \cdots a_{k}} \circ \gamma(\nu) \xi\right) e^{a_{1} \cdots a_{k}} \\
& \in \Omega^{k}(M), \quad \forall a_{1}, \ldots, a_{k}=1 \cdots 8, \quad \forall k=0 \cdots 8,
\end{aligned}
$$

which, upon using $\epsilon_{\mathscr{B}_{-}}=-1$ and $\sigma_{\mathscr{B}_{-}}=+1$ and the wellknown formula,

$$
\gamma_{a_{1} \cdots a_{k}}=\frac{(-1)^{k(k-1) / 2}}{(d-k) !} \epsilon_{a_{1} \cdots a_{k}}^{a_{k+1} \cdots a_{d}} \gamma_{a_{k+1} \cdots a_{d}} \circ \gamma^{(d+1)}
$$

would lead to the homogeneous form-valued bilinears:

$$
\begin{aligned}
\check{\mathbf{E}}_{-}^{(0)} & =\mathscr{B}_{-}(\xi, \xi)=\lambda=\mathscr{B}(\xi, \gamma(\nu) \xi)=\check{\mathbf{E}}^{(8)} \diamond \nu, \\
\check{\mathbf{E}}_{-}^{(3)} & =-\frac{1}{3 !} \mathscr{B}_{-}\left(\xi, \gamma_{a_{1} \cdots a_{3}} \xi\right) e^{a_{1} \cdots a_{3}} \\
& =-\frac{1}{3 !} \mathscr{B}\left(\xi, \gamma_{a_{1} \cdots a_{3}} \circ \gamma(\nu) \xi\right) e^{a_{1} \cdots a_{3}}=-\check{\mathbf{E}}^{(5)} \diamond \nu, \\
\check{\mathbf{E}}_{-}^{(4)} & =\frac{1}{4 !} \mathscr{B}_{-}\left(\xi, \gamma_{a_{1} \cdots a_{4}} \xi\right) e^{a_{1} \cdots a_{4}} \\
& =\frac{1}{4 !} \mathscr{B}\left(\xi, \gamma_{a_{1} \cdots a_{4}} \circ \gamma(\nu) \xi\right) e^{a_{1} \cdots a_{4}}=\check{\mathbf{E}}^{(4)} \diamond \nu, \\
\check{\mathbf{E}}_{-}^{(7)} & =-\frac{1}{7 !} \mathscr{B}-\left(\xi, \gamma_{a_{1} \cdots a_{7}} \xi\right) e^{a_{1} \cdots a_{7}} \\
& =-\frac{1}{7 !} \mathscr{B}\left(\xi, \gamma_{a_{1} \cdots a_{7}} \circ \gamma(\nu) \xi\right) e^{a_{1} \cdots a_{7}}=-\check{\mathbf{E}}^{(1)} \diamond \nu, \\
\check{\mathbf{E}}_{-}^{(8)} & =\frac{1}{8 !} \mathscr{B} \mathscr{B}_{-}\left(\xi, \gamma_{a_{1} \cdots a_{8}} \xi\right) e^{a_{1} \cdots a_{8}}=\beta \nu \\
& =\frac{1}{8 !} \mathscr{B}\left(\xi, \gamma_{a_{1} \cdots a_{8}} \circ \gamma(\nu) \xi\right) e^{a_{1} \cdots a_{8}}=\check{\mathbf{E}}^{(0)} \diamond \nu .
\end{aligned}
$$

Thus, one could also write the generator:

$$
\check{E}_{-}=\frac{N}{2^{d}} \sum_{k} \check{\mathbf{E}}_{-}^{(k)}
$$

which would lead to the same result up to twisted Hodge duality-since the form-valued bilinears constructed using $\mathscr{B}_{\text {- }}$ are connected through Hodge duality with those constructed using $\mathscr{B}_{+}$.

Following the notations and conventions of [3], we define

$$
\begin{gathered}
a \stackrel{\text { def }}{=} \frac{1}{2} \check{\mathbf{E}}^{(0)}, \\
\bar{K} \stackrel{\text { def }}{=} \frac{1}{2} \check{\mathbf{E}}^{(1)}, \\
Y \stackrel{\text { def }}{=} \frac{1}{2} \check{\mathbf{E}}^{(4)}, \\
\bar{Z} \stackrel{\text { def }}{=} \frac{1}{2} \check{\mathbf{E}}^{(5)}, \\
W \stackrel{\text { def }}{=} \frac{1}{2} \check{\mathbf{E}}^{(8)}=b \nu,
\end{gathered}
$$

where $2 a$ is the squared norm of $\xi$ and $b$ a smooth function on $M$. With these notations, (314) becomes

$$
\check{E}=\frac{2}{16}[a+\bar{K}+Y+\bar{Z}+b v] .
$$

Note that we use the five-form $\bar{Z}$ instead of its sign-reversed Hodge dual $\bar{\phi} \stackrel{\text { def }}{=}-* \bar{Z}$, which was used in [3]. We prefer to work with $\xi=\xi_{+} \oplus \xi_{-}$rather than with $\xi_{ \pm}$and $\epsilon^{ \pm} \stackrel{\text { def }}{=}$ $(1 / \sqrt{2})\left(\xi_{+} \pm \xi_{-}\right)$, which were used in loc. cit. All in all, we 
have the following correspondence with the notations and conventions of [3]:

$$
\begin{aligned}
\xi_{ \pm} & =\mathscr{P}_{ \pm} \xi, \quad \text { where } \mathscr{P}_{ \pm}=\frac{1}{2}\left(1 \pm \gamma^{(9)}\right), \\
\varepsilon^{+} & =\frac{1}{\sqrt{2}} \xi, \\
\varepsilon^{-} & =\frac{1}{\sqrt{2}} \gamma^{(9)} \xi, \\
\bar{K}_{m} & =\xi_{+}^{t} \gamma_{m} \xi_{-}=\xi^{t} \mathscr{P}_{+} \gamma_{m} \xi=\frac{1}{2} \xi^{t} \gamma_{m} \xi, \\
-(* \bar{Z})_{m n p} & =\bar{\phi}_{m n p}=\xi_{+}^{t} \gamma_{m n p} \xi_{-}=\xi^{t} \mathscr{P}_{+} \gamma_{m n p} \xi \\
& =\frac{1}{2} \xi^{t} \gamma^{(9)} \gamma_{m n p} \xi, \\
Y_{m n p r} & =\varepsilon^{ \pm t} \gamma_{m n p r} \varepsilon^{ \pm} \\
& =\frac{1}{2}\left(\xi_{+}^{t} \gamma_{m n p r} \xi_{+}+\xi_{-}^{t} \gamma_{m n p r} \xi_{-}\right) \\
& =\frac{1}{2} \xi^{t} \gamma_{m n p r} \xi .
\end{aligned}
$$

The dequantizations of $A_{m}$ and $Q$ are given by

$$
\begin{aligned}
\check{A}_{m} \stackrel{\text { def }}{=} \gamma^{-1}\left(A_{m}\right)= & \frac{1}{4} l_{\left(e_{m}\right)_{\sharp}} F+\frac{1}{4}\left(\left(e_{m}\right)_{\sharp} \wedge f\right) \diamond \nu \\
& +\kappa\left(e_{m}\right)_{\sharp} \diamond \nu, \\
\check{Q} \stackrel{\text { def }}{=} \gamma^{-1}(Q)= & \frac{1}{2} \mathrm{~d} \Delta-\frac{1}{6} f \diamond \nu-\frac{1}{12} F-\kappa \nu .
\end{aligned}
$$

As shown in Section 5, the CGK pinor equations imply the following conditions for $\check{E}$ :

$$
\begin{aligned}
\check{Q} \diamond \check{E} & =0, \\
\nabla_{m} \check{E} & =-\left[\check{A}_{m}, \check{E}\right]_{-, \diamond} .
\end{aligned}
$$

In turn, relations (324) imply

$$
\mathrm{d} \check{E}=-\left(e_{m}\right)_{\sharp} \wedge\left[\check{A}_{m}, \check{E}\right]_{-, \diamond} .
$$

In this case, (277) amounts to only one quadratic relation for the inhomogeneous form $\check{E}$ :

$$
\check{E} \diamond \check{E}=2 a \check{E},
$$

which encodes the relevant Fierz identities between the form bilinears constructed from $\xi$.

We remind the reader of the following relations (see the previous sections):

$$
\begin{aligned}
* \omega & =\tau(\omega) \diamond \nu=\iota_{\omega} \nu, \\
* * \omega & =\pi(\omega),
\end{aligned}
$$

where $\tau$ is the reversion defined in (41). During our calculations we will use the following identities which hold in any dimension and signature for any homogeneous forms $\omega \epsilon$ $\Omega^{r}(M)$ and $\eta \in \Omega^{s}(M)$

$$
\begin{aligned}
& \omega \wedge * \eta=(-1)^{r(s-1)} * \iota_{\tau(\omega)} \eta, \quad \text { when } r \leq s, \\
& \iota_{\omega}(* \eta)=(-1)^{r s} *(\tau(\omega) \wedge \eta), \quad \text { when } r+s \leq d
\end{aligned}
$$

and of the following:

$$
\begin{aligned}
& (-1)^{[(m+1) / 2]}\left[\pi^{m}(\omega)\right] \triangle_{m}[* \tau(\omega)] \\
& =(-1)^{\left[\left(m^{\prime}+1\right) / 2\right]}[* \tau(\omega)] \triangle_{m^{\prime}}\left[\pi^{d-1}(\eta)\right] \\
& =(-1)^{\left[\left(m^{\prime \prime}+1\right) / 2\right]} * \tau\left[\pi^{m^{\prime \prime}}(\omega) \triangle_{m^{\prime \prime}} \eta\right],
\end{aligned}
$$

for $\tilde{\omega}-m=\tilde{\eta}-m^{\prime}=m^{\prime \prime}$, where $m, m^{\prime}, m^{\prime \prime}>0$.

We also remind the reader that one can uniquely decompose any $k$-form $\omega \in \Omega^{k}(M)$ into parallel and orthogonal parts with respect to any fixed 1-form $\theta \in \Omega^{1}(M)$ such that $\omega=$ $\omega_{\perp}+\omega_{\|}$, where $\omega_{\|}=\theta \wedge \omega_{\mathrm{T}}$ with $\omega_{\mathrm{T}} \in \Omega^{k-1}(M), \omega_{\perp} \in \Omega^{k}(M)$, and $\theta \triangle_{1} \omega_{\top}=\theta \triangle_{1} \omega_{\perp}=0$ (see Section 3.5). Choosing $\theta=\bar{K}$, one has $Y=Y_{\perp}+Y_{\|}$and $\bar{Z}=\bar{Z}_{\perp}+\bar{Z}_{\|}$. Note that $\nu=\nu_{\|}$and $\bar{K}=\bar{K}_{\|}$.

Given the decomposition of $Y$, its Hodge dual must take the form $* Y=*\left(Y_{\perp}\right)+*\left(Y_{\|}\right)=\alpha_{1} Y_{\|}+\alpha_{2} Y_{\perp}$ for some $\alpha_{1}, \alpha_{2} \in C^{\infty}(M, \mathbb{R})$, since the Hodge dual of any component parallel to $\theta$ is orthogonal to $\theta$ while the Hodge dual of any component orthogonal to $\theta$ is parallel to $\theta$ and since there are no other four-form-valued pinor bilinears that could appear in the right hand side. In the current example (see Table 1), the volume form $v$ is twisted central (i.e., we have $v \diamond \omega=$ $\pi(\omega) \diamond \nu$ for any inhomogeneous differential form $\omega$ ) and satisfies $\nu \diamond \nu=+1$. The latter property amounts to $* * Y=Y$, which leads to $\alpha_{2}=1 / \alpha_{1}$ and implies

$$
* Y=\alpha_{1} Y_{\|}+\frac{1}{\alpha_{1}} Y_{\perp}, \quad \text { with } \alpha_{1} \in C^{\infty}(M, \mathbb{R})
$$

Expanding (323) into rank components gives the following conditions, which are equivalent to the "useful relations" discussed in Appendix C of [3]:

$$
\begin{aligned}
& -\frac{1}{6} \iota_{F} Y+\iota_{\mathrm{d} \Delta} \bar{K}-2 \kappa b=0, \\
& -\frac{1}{6} \iota_{F} \bar{Z}-\frac{b}{3} f+a \mathrm{~d} \Delta=0, \\
& \mathrm{~d} \Delta \wedge \bar{K}-\frac{1}{6} F \triangle_{3} Y+\frac{1}{3} \iota_{f} * \bar{Z}=0, \\
& -\frac{1}{3} \iota_{f} * Y+2 \kappa * \bar{Z}+\frac{1}{6} \iota_{\bar{K}} F+\iota_{\mathrm{d} \Delta} Y-\frac{1}{6} F \triangle_{3} \bar{Z}=0,
\end{aligned}
$$




$$
\begin{aligned}
& \frac{1}{6} F \triangle_{2} Y-2 \kappa * Y+\frac{1}{3} f \wedge * \bar{Z}-\frac{b}{6} * F+\iota_{\mathrm{d} \Delta} \bar{Z}-\frac{a}{6} F \\
& \quad=0 \\
& -\frac{1}{6} F \wedge \bar{K}+\mathrm{d} \Delta \wedge Y+\frac{1}{6} F \triangle_{2} \bar{Z}-\frac{1}{3} f \wedge * Y=0, \\
& \mathrm{~d} \Delta \wedge \bar{Z}+\frac{1}{3} \iota_{f} * \bar{K}+\frac{1}{6} F \triangle_{1} Y=0 \\
& -\frac{a}{3} * f+2 \kappa * \bar{K}+\frac{1}{6} F \triangle_{1} \bar{Z}+b *(\mathrm{~d} \Delta)=0, \\
& 2 a \kappa \nu-\frac{1}{3} f \wedge * \bar{K}+\frac{1}{6} Y \wedge F=0 .
\end{aligned}
$$

Remark 15. It will be useful to Hodge dualize relations (331), which gives

$$
\begin{aligned}
& -\frac{1}{6} F \wedge * Y+\mathrm{d} \Delta \wedge * \bar{K}-2 \kappa b v=0 \\
& -\frac{1}{6} F \wedge * \bar{Z}-\frac{b}{3} * f+a * \mathrm{~d} \Delta=0 \\
& -\iota_{\mathrm{d} \Delta} * \bar{K}-\frac{1}{6} *\left(F \triangle_{3} Y\right)-\frac{1}{3} f \wedge \bar{Z}=0 \\
& \frac{1}{3} f \wedge Y-2 \kappa \bar{Z}-\frac{1}{6} \bar{K} \wedge * F-\mathrm{d} \Delta \wedge * Y \\
& \quad-\frac{1}{6} *\left(F \triangle_{3} \bar{Z}\right)=0, \\
& \frac{1}{6} *\left(F \triangle_{2} Y\right)-2 \kappa Y+\frac{1}{3} \iota_{f} \bar{Z}-\frac{b}{6} F+\mathrm{d} \Delta \wedge * \bar{Z} \\
& \quad-\frac{a}{6} * F=0 \\
& -\frac{1}{6} l_{\bar{K}} * F+\iota_{\mathrm{d} \Delta} * Y-\frac{1}{6} *\left(\bar{F} \triangle_{2} Z\right)-\frac{1}{3} \iota_{f} Y=0 \\
& -\frac{1}{6} *\left(F \triangle_{1} Y\right)-\iota_{\mathrm{d} \Delta} * \bar{Z}-\frac{1}{3} f \wedge \bar{K}=0 \\
& -\frac{a}{3} f+2 \kappa \bar{K}+\frac{1}{6} \iota_{F} F+b \mathrm{~d} \Delta=0 \\
& -\frac{1}{3} \iota_{f} \bar{K}+\frac{1}{6} l_{Y} * F+2 \kappa a=0,
\end{aligned}
$$

where we used (328) and (329).

Similarly, the rank expansion of (324) gives

$$
\begin{aligned}
\partial_{m} b= & 2 \kappa \iota_{\left(e_{m}\right)_{\sharp}} \bar{K}-\frac{1}{2} *\left[\left(\iota_{\left(e_{m}\right)_{\sharp}} F\right) \wedge \bar{Z}\right], \\
\nabla_{m} \bar{K}= & -2 \kappa b\left(e_{m}\right)_{\sharp}-\frac{1}{2} *\left(\left(e_{m}\right)_{\sharp} \wedge f \wedge \bar{Z}\right) \\
& +\frac{1}{2}\left(\iota_{\left(e_{m}\right)_{\sharp}} F\right) \triangle_{3} Y,
\end{aligned}
$$

$$
\begin{aligned}
\nabla_{m} Y= & 2 \kappa\left(e_{m}\right)_{\sharp} \wedge * \bar{Z}+\frac{1}{2}\left(\left(e_{m}\right)_{\sharp} \wedge f\right) \triangle_{1} * Y \\
& +\frac{1}{2} \bar{K} \wedge\left(\iota_{\left(e_{m}\right)_{\sharp}} F\right)+\frac{1}{2}\left(\iota_{\left(e_{m}\right)_{\sharp}} F\right) \triangle_{2} \bar{Z}, \\
\nabla_{m} Z= & -2 \kappa\left(e_{m}\right)_{\sharp} \wedge * Y+\frac{1}{2} *\left(\left(e_{m}\right)_{\sharp} \wedge \bar{K} \wedge f\right) \\
& +\frac{1}{2}\left(e_{m}\right)_{\sharp} \wedge f \wedge * \bar{Z}-\frac{1}{2}\left(\iota_{\left(e_{m}\right)_{\sharp}} F\right) \triangle_{1} Y \\
& -\frac{b}{2}\left(e_{m}\right)_{\sharp} \wedge * F,
\end{aligned}
$$

which in turn implies the following constraints representing the rank components of (325):

$$
\begin{aligned}
\mathrm{d} b & =2 \kappa \bar{K}+\frac{1}{2} \iota_{* \bar{Z}} F, \\
\mathrm{~d} \bar{K} & =-\frac{1}{2} F \triangle_{3} Y+\iota_{f} * \bar{Z}, \\
\mathrm{~d} Y & =F \triangle_{2} \bar{Z}-2 f \wedge * Y-2 F \wedge \bar{K}, \\
\mathrm{~d} \bar{Z} & =\frac{3}{2} F \triangle_{1} Y+3 \iota_{f} * \bar{K} .
\end{aligned}
$$

The Hodge duals of (333)

$$
\begin{aligned}
\partial_{m} b v= & 2 \kappa\left(e_{m}\right)_{\sharp} \wedge * \bar{K}-\frac{1}{2}\left(\iota_{\left(e_{m}\right)_{\sharp}} F\right) \wedge \bar{Z} \\
\nabla_{m} * \bar{K}= & -2 \kappa b *\left(e_{m}\right)_{\sharp}+\frac{1}{2}\left(e_{m}\right)_{\sharp} \wedge f \wedge \bar{Z} \\
& -\frac{1}{2}\left(\iota_{\left(e_{m}\right)_{\sharp}} F\right) \wedge * Y \\
\nabla_{m} * Y= & 2 \kappa \iota_{\left(e_{m}\right)_{\sharp}} \bar{Z}+\frac{1}{2}\left(\left(e_{m}\right)_{\sharp} \wedge f\right) \triangle_{1} Y \\
& +\frac{1}{2} \iota_{\bar{K}}\left(\left(e_{m}\right)_{\sharp} \wedge * F\right) \\
& -\frac{1}{2}\left(\iota_{\left(e_{m}\right)_{\sharp}} F\right) \triangle_{1} * \bar{Z} \\
\nabla_{m} * \bar{Z}= & -2 \kappa \iota_{\left(e_{m}\right)_{\sharp}} Y-\frac{1}{2}\left(e_{m}\right)_{\sharp} \wedge \bar{K} \wedge f \\
& +\frac{1}{2} \iota_{\left(\left(e_{m}\right)_{\sharp} \wedge f\right)} \bar{Z}+\frac{1}{2}\left(\iota_{\left(e_{m}\right)_{\sharp}} F\right) \triangle_{2} * Y \\
& -\frac{1}{2} b \iota_{\left(e_{m}\right)_{\sharp}} F
\end{aligned}
$$

lead to another set of differential constraints:

$$
\begin{aligned}
\mathrm{d}(* \bar{K})= & -16 \kappa b \nu-2 F \wedge * Y \\
\mathrm{~d}(* Y)= & 10 \kappa \bar{Z}-2 f \wedge Y+\frac{1}{2} \bar{K} \wedge * F \\
& +\frac{3}{2} *\left(F \triangle_{3} \bar{Z}\right) \\
\mathrm{d}(* \bar{Z})= & -2 b F-8 \kappa Y+*\left(F \triangle_{2} Y\right)+2 \iota_{f} \bar{Z}
\end{aligned}
$$


Finally, expanding (326) and using the properties of $v$, we find

$$
\begin{gathered}
\bar{K} \diamond \bar{K}+\bar{K} \diamond Y+Y \diamond \bar{K}+\bar{K} \diamond \bar{Z}+\bar{Z} \diamond \bar{K}+Y \diamond Y \\
+\bar{Z} \diamond \bar{Z}+Y \diamond \bar{Z}+\bar{Z} \diamond Y+2 b * Y+b^{2} \\
=15 a^{2}+14 a \bar{K}+14 a Y+14 a \bar{Z}+14 a b v .
\end{gathered}
$$

We write below the expansions for all those geometric products (see (35)) that appear in (337). Let us first list the geometric products involving $\bar{K}$ :

$$
\begin{aligned}
& \bar{K} \diamond \bar{K}=\|\bar{K}\|^{2}, \\
& \bar{K} \diamond Y=\bar{K} \wedge Y_{\perp}+\bar{K} \triangle_{1} Y_{\|}, \\
& Y \diamond \bar{K}=\bar{K} \wedge Y_{\perp}-\bar{K} \triangle_{1} Y_{\|}, \\
& \bar{K} \diamond \bar{Z}=\bar{K} \wedge \bar{Z}_{\perp}+\bar{K} \triangle_{1} \bar{Z}_{\|}=\bar{K} \triangle_{1} \bar{Z}, \\
& \bar{Z} \diamond \bar{K}=-\bar{K} \wedge \bar{Z}_{\perp}+\bar{K} \triangle_{1} \bar{Z}_{\|}=\bar{K} \triangle_{1} \bar{Z} .
\end{aligned}
$$

Notice that $\bar{K} \wedge \bar{Z}_{\perp}=0$, since there is no nontrivial six-form pinor bilinear that can be constructed in this case. Thus, we must have $\bar{Z}_{\perp}=0$, which means $\bar{Z}=\bar{Z}_{\|}$, a relation which we use when performing the following expansions:

$$
\begin{aligned}
Y \diamond Y= & 2 Y_{\|} \wedge Y_{\perp}-Y_{\|} \triangle_{2} Y_{\|}-2 Y_{\|} \triangle_{2} Y_{\perp} \\
& -Y_{\perp} \triangle_{2} Y_{\perp}+\left\|Y_{\|}\right\|^{2}+\left\|Y_{\perp}\right\|^{2}, \\
Y \diamond \bar{Z}= & -Y_{\|} \triangle_{1} \bar{Z}_{\|}-Y_{\perp} \triangle_{2} \bar{Z}_{\|}+Y_{\|} \triangle_{3} \bar{Z}_{\|} \\
& +Y_{\perp} \triangle_{4} \bar{Z}_{\|}, \\
\bar{Z} \diamond Y= & Y_{\|} \triangle_{1} \bar{Z}_{\|}-Y_{\perp} \triangle_{2} \bar{Z}_{\|}-Y_{\|} \triangle_{3} \bar{Z}_{\|} \\
& +Y_{\perp} \triangle_{4} \bar{Z}_{\|}, \\
\bar{Z} \diamond \bar{Z}= & \bar{Z}_{\|} \triangle_{1} \bar{Z}_{\|}-\bar{Z}_{\|} \triangle_{3} \bar{Z}_{\|}+\|\bar{Z}\|^{2} .
\end{aligned}
$$

We mention here that expansions (331)-(339) were obtained using a package of procedures which we created using Ricci [33]. We have also verified these relations (in tensorial form) using Cadabra [34].

We deduce from the Fierz identities in normal cases that any generalized product of form-valued pinor bilinears can be expressed as a form-valued pinor bilinear. In (339), we omitted to write down those terms which are tautologically zero due to the graded antisymmetry of the wedge product. We also omitted writing some other vanishing terms such as $Y_{\perp} \wedge Y_{\perp}$, which has rank 8 and thus must be proportional to the volume form but vanishes since the volume form does not have an orthogonal component. Similarly, $Y_{\|} \triangle_{1} \bar{Z}_{\|}=0$ since it should be a parallel 7-form and thus proportional to $(* \bar{K})_{\|}=*\left(\bar{K}_{\perp}\right)$, but $\bar{K}_{\perp}=0$. Furthermore, $Y_{\perp} \triangle_{1} \bar{Z}_{\|}=0$ since it is an orthogonal 5-form, thus proportional to $\bar{Z}_{\perp}=0$. For similar reasons, $Y_{\perp} \triangle_{3} \bar{Z}_{\|}=0$ and $Y_{\|} \triangle_{4} \bar{Z}_{\|}=0$.
Given expansions (338) and (339), identity (337) gives the following relations when separated into rank components:

$$
\begin{aligned}
& \|\bar{K}\|^{2}+\left\|Y_{\perp}\right\|^{2}+\left\|Y_{\|}\right\|^{2}+\|\bar{Z}\|^{2}+b^{2}=15 a^{2}, \\
& Y_{\perp} \triangle_{4} \bar{Z}=7 a \bar{K} \\
& 2 \bar{K} \triangle_{1} \bar{Z}-Y_{\|} \triangle_{2} Y_{\|}-2 Y_{\|} \triangle_{2} Y_{\perp}-Y_{\perp} \triangle_{2} Y_{\perp} \\
& \quad-\bar{Z} \triangle_{3} \bar{Z}+2 b * Y=14 a Y, \\
& \bar{K} \wedge Y_{\perp}-Y_{\perp} \triangle_{2} \bar{Z}=7 a \bar{Z} \\
& 2 Y_{\|} \wedge Y_{\perp}+\bar{Z} \triangle_{1} \bar{Z}=14 a b \nu .
\end{aligned}
$$

In order to solve system (340), we introduce the notations:

$$
\begin{aligned}
& \bar{K} \wedge Y_{\perp}=e \bar{Z}, \\
& \bar{K} \triangle_{1} Y_{\|}=f * \bar{Z}, \\
& \bar{K} \triangle_{1} \bar{Z}=g Y_{\perp}, \\
& Y_{\|} \wedge Y_{\perp}=h v, \\
& Y_{\|} \triangle_{2} Y_{\|}=y_{1} Y_{\perp}, \\
& Y_{\|} \triangle_{2} Y_{\perp}=y_{2} Y_{\|}, \\
& Y_{\perp} \triangle_{2} Y_{\perp}=y_{3} Y_{\perp}, \\
& Y_{\|} \triangle_{1} \bar{Z}=n * \bar{K}, \\
& Y_{\perp} \triangle_{2} \bar{Z}=r \bar{Z}, \\
& Y_{\|} \triangle_{3} \bar{Z}=s * \bar{Z}, \\
& Y_{\perp} \triangle_{4} \bar{Z}=t \bar{K}, \\
& \bar{Z} \triangle_{1} \bar{Z}=u v, \\
& \bar{Z} \triangle_{3} \bar{Z}=x Y_{\perp},
\end{aligned}
$$

where $e, f, g, h, y_{1}, y_{2}, y_{3}, n, r, s, t, u, x$ are smooth functions on $M$ which we want to determine in terms of $a$ and $b$.

Using (341), system (340) leads to four independent relations for ranks $0,1,5,8$ and two independent identities for the parallel and perpendicular components of rank 4:

$$
\begin{aligned}
b^{2}+\|\bar{K}\|^{2}+\left\|Y_{\|}\right\|^{2}+\left\|Y_{\perp}\right\|^{2}+\|\bar{Z}\|^{2} & =15 a^{2}, \\
t & =7 a, \\
e-r & =7 a, \\
2 h+u & =14 a b, \\
b \alpha_{1}-y_{2} & =7 a, \\
\frac{2 b}{\alpha_{1}}+2 g-\left(y_{1}+y_{3}\right)-x & =14 a .
\end{aligned}
$$


Using associativity of the geometric product

$$
\begin{aligned}
& (\bar{K} \diamond \bar{K}) \diamond Y=\bar{K} \diamond(\bar{K} \diamond Y), \\
& (\bar{K} \diamond Y) \diamond Y=\bar{K} \diamond(Y \diamond Y), \\
& (\bar{K} \diamond \bar{Z}) \diamond \bar{Z}=\bar{K} \diamond(\bar{Z} \diamond \bar{Z}), \\
& (\bar{K} \diamond Y) \diamond \bar{Z}=\bar{K} \diamond(Y \diamond \bar{Z}), \\
& (Y \diamond Y) \diamond \bar{Z}=Y \diamond(Y \diamond \bar{Z}), \\
& (\bar{K} \diamond \bar{Z}) \diamond Y=\bar{K} \diamond(\bar{Z} \diamond Y)
\end{aligned}
$$

as well as (338), (339), and (341), we find the relations

$$
\begin{aligned}
& \|\bar{K}\|^{2}=e g, \\
& e=f \alpha_{1} \\
& e n+f t=2 h \text {, } \\
& e r+f s=e\left(y_{1}+y_{3}\right), \\
& e s+r f=2 y_{2} f \text {, } \\
& \left\|Y_{\|}\right\|^{2}+\left\|Y_{\perp}\right\|^{2}=e t+f n, \\
& \|\bar{Z}\|^{2}=t g, \\
& u=0 \text {, } \\
& g r=x e, \\
& f x=s g, \\
& f\|\bar{Z}\|^{2}=n\|\bar{K}\|^{2}, \\
& e\|\bar{Z}\|^{2}=t\|\bar{K}\|^{2} \text {, } \\
& 2 h+2 s y_{2}=n e+2 r s+f t, \\
& \left\|Y_{\|}\right\|^{2}+\left\|Y_{\perp}\right\|^{2}+r\left(y_{1}+y_{3}\right)=n f+r^{2}+s^{2}+t e, \\
& t\left(y_{1}+y_{3}\right)=r t+n s, \\
& n\|\bar{K}\|^{2}=g h, \\
& g\left\|Y_{\perp}\right\|^{2}=t\|\bar{K}\|^{2}, \\
& 2 y_{2} n=n r+t s \\
& y_{3}=r \text {, } \\
& y_{2}=s \alpha_{1}
\end{aligned}
$$

which need not be independent. We thus find that the Fierz identities are equivalent to the algebraic system of (342)(344), which can be solved using Mathematica ${ }^{\circledR}$ and give

$$
\begin{aligned}
& e=a, \\
& f=b \text {, } \\
& g=\frac{a^{2}-b^{2}}{a} \\
& h=7 a b \text {, } \\
& y_{1}=-\frac{6 b^{2}}{a}, \\
& y_{2}=-6 a, \\
& y_{3}=-6 a, \\
& n=7 b \text {, } \\
& r=-6 a, \\
& s=-6 b, \\
& t=7 a, \\
& u=0 \text {, } \\
& x=-\frac{6\left(a^{2}-b^{2}\right)}{a}, \\
& \alpha_{1}=\frac{a}{b}, \\
& \|\bar{K}\|^{2}=a^{2}-b^{2} \text {, } \\
& \left\|Y_{\|}\right\|^{2}=7 b^{2}, \\
& \left\|Y_{\perp}\right\|^{2}=7 a^{2}, \\
& \|\bar{Z}\|^{2}=7\left(a^{2}-b^{2}\right) \text {. }
\end{aligned}
$$

Substituting this solution into (341) gives

$$
\begin{aligned}
\bar{Z} & =\frac{1}{a} \bar{K} \wedge Y_{\perp}, \\
* \bar{Z} & =\frac{1}{b} \bar{K} \triangle_{1} Y_{\|}, \\
* \bar{K} & =\frac{1}{7 b} Y_{\|} \triangle_{1} \bar{Z}, \\
Y_{\|} \wedge Y_{\perp} & =7 a b v, \\
Y_{\|} \triangle_{2} Y_{\|} & =-\frac{6 b^{2}}{a} Y_{\perp}, \\
Y_{\|} \triangle_{2} Y_{\perp} & =-6 a Y_{\|}, \\
Y_{\perp} \triangle_{2} Y_{\perp} & =-6 a Y_{\perp}, \\
* Y & =\frac{a}{b} Y_{\|}+\frac{b}{a} Y_{\perp},
\end{aligned}
$$




$$
\begin{aligned}
\|\bar{K}\|^{2} & =a^{2}-b^{2}, \\
\left\|Y_{\|}\right\|^{2} & =7 b^{2}, \\
\left\|Y_{\perp}\right\|^{2} & =7 a^{2}, \\
\|\bar{Z}\|^{2} & =7\left(a^{2}-b^{2}\right), \\
Y & =Y_{\|}+Y_{\perp}=\frac{b}{\|\bar{K}\|^{2}} \bar{K} \wedge \bar{Z}+\frac{a}{\|\bar{K}\|^{2}} l_{\bar{K}} \bar{Z}, \\
\bar{Z} \triangle_{3} \bar{Z} & =-6 l_{\bar{K}} \bar{Z} .
\end{aligned}
$$

Taking (as in [3]) $a=1$ and $b=\sin \zeta$ (since $1-b^{2}>0$ for the norms to be positive) we find agreement with the results in loc. cit. (up to a sign issue which is discussed in [35]), as for example,

$$
\begin{aligned}
\|K\|^{2} & =1, \\
\|* Z\|^{2} & =7, \\
\text { where } K \stackrel{\text { def }}{=}(\cos \zeta)^{-1} \bar{K}, & \stackrel{\text { def }}{=}(\cos \zeta)^{-1} \bar{Z}, \\
\iota_{K}(* Z) & =0, \\
Y & =\iota_{K} Z-(* Z) \wedge K \sin \zeta, \\
\mathrm{d}\left(e^{3 \Delta} \bar{K}\right) & =0, \\
K \wedge \mathrm{d}\left(e^{6 \Delta} \iota_{K} Z\right) & =0, \\
e^{-6 \Delta} \mathrm{d}\left(e^{6 \Delta} * \bar{Z}\right) & =* F-F \sin \zeta+4 \kappa Y, \\
e^{-3 \Delta} \mathrm{d}\left(e^{3 \Delta} \sin \zeta\right) & =f-4 \kappa \bar{K}, \\
e^{-12 \Delta} \mathrm{d}\left(e^{12 \Delta} \operatorname{vol}_{7} \cos \zeta\right) & =-8 \kappa \operatorname{vol}_{7} \wedge K \sin \zeta,
\end{aligned}
$$

with vol $_{7}$ defined as in [3]:

$$
\begin{aligned}
\operatorname{vol}_{7} & =\frac{1}{7} \phi \wedge \iota_{K} * \phi=-\frac{1}{7}(* Z) \wedge \iota_{K} Z=-\iota_{K} \nu \\
& =-* K .
\end{aligned}
$$

It is easy to see that the first four of these conditions follow directly from the Fierz identities (346), while the last four can be obtained using the algebraic constraints (331) and the differential constraints (334).

Proof of Relations (347) and (348). From (346), we have

$$
\begin{aligned}
& \|\bar{K}\|^{2}=a^{2}-b^{2}, \\
& \|\bar{Z}\|^{2}=7\left(a^{2}-b^{2}\right) .
\end{aligned}
$$

For $a=1, b=\sin \zeta, K=(\cos \zeta)^{-1} \bar{K}$, and $Z=(\cos \zeta)^{-1} \bar{Z}$, this result becomes

$$
\begin{aligned}
\|K\|^{2} & =1, \\
\|* Z\|^{2} & =\|Z\|^{2}=7 .
\end{aligned}
$$

Proof of Relation (349). The second relation in (328) gives $\iota_{K}(* Z)=-*(K \wedge Z)$, where $K \wedge Z=\left(1 / \cos ^{2} \zeta\right) \bar{K} \wedge \bar{Z}$ must be a 6-form bilinear in $\xi$ since $\bar{K}$ and $\bar{Z}$ are bilinears in $\xi$ and since the Fierz identities allow us to reduce $\bar{K} \wedge \bar{Z}$ to a pinor bilinear. But there is no nontrivial 6-form pinor bilinear which can be constructed in our case, and thus we must have $\bar{K} \wedge \bar{Z}=0$. This proves relation (349).

Proof of Relation (350). Starting from the expression $Y=$ $\left(b /\|\bar{K}\|^{2}\right) \bar{K} \wedge * \bar{Z}+\left(a /\|\bar{K}\|^{2}\right) \iota \bar{K} \bar{Z}$ found in (346), taking the normalization used in $[3,4]$ and the notations in (347), (348), one finds

$$
\begin{aligned}
Y & =\frac{1}{\cos ^{2} \zeta}\left(\sin \zeta \bar{K} \wedge * \bar{Z}+\iota_{\bar{K}} \bar{Z}\right) \\
& =\iota_{K} Z-(* Z) \wedge K \sin \zeta,
\end{aligned}
$$

thus proving relation (350).

Proof of Relation (351). The third algebraic constraint listed in (331)

$$
\begin{aligned}
\mathrm{d} \Delta \wedge \bar{K}-\frac{1}{6} F \triangle_{3} Y+\frac{1}{3} \iota_{f} * \bar{Z} & =0 \Longleftrightarrow \\
3 \mathrm{~d} \Delta \wedge \bar{K} & =\frac{1}{2} F \triangle_{3} Y-\iota_{f} * \bar{Z}
\end{aligned}
$$

and the second differential constraint listed in (334)

$$
\mathrm{d} \bar{K}=-\frac{1}{2} F \triangle_{3} Y+\iota_{f} * \bar{Z}
$$

imply the relation

$$
3 \mathrm{~d} \Delta \wedge \bar{K}+\mathrm{d} \bar{K}=0 \Longleftrightarrow \mathrm{d} \bar{K}=3 \bar{K} \wedge \mathrm{d} \Delta,
$$

which is easily seen to be equivalent to (351).

Proof of Relation (352). Using (350), one can write (352) in the equivalent form:

$$
K \wedge[6 \mathrm{~d} \Delta \wedge Y+\mathrm{d} Y-\sin \zeta(* Z) \wedge \mathrm{d} K]=0,
$$

where we used $K \wedge K=0$. Relations (362) and $K \wedge \bar{K}=0$ imply $K \wedge(\sin \zeta(* Z) \wedge \mathrm{d} K)=0$, so (352) reduces to

$$
K \wedge(6 \mathrm{~d} \Delta \wedge Y+\mathrm{d} Y)=0 .
$$

To prove (364), notice that adding the sixth algebraic constraint listed in (331)

$$
6 \mathrm{~d} \Delta \wedge Y=F \wedge \bar{K}-F \triangle_{2} \bar{Z}+2 f \wedge * Y
$$


to the third differential constraint listed in (334)

$$
\mathrm{d} Y=-2 F \wedge \bar{K}+F \triangle_{2} \bar{Z}-2 f \wedge * Y
$$

gives

$$
6 \mathrm{~d} \Delta \wedge Y+\mathrm{d} Y=-F \wedge \bar{K}
$$

which in turn implies (364) upon using the identity $K \wedge \bar{K}=$ 0 .

Proof of Relation (353). Relation (353) is equivalent to

$$
6 \mathrm{~d} \Delta \wedge(* \bar{Z})+\mathrm{d}(* \bar{Z})=* F-F \sin \zeta+4 \kappa Y .
$$

To prove (368), notice that the fifth relation in (332), which is the Hodge dual of the fifth algebraic relation listed in (331), when multiplied by 6 gives

$$
\begin{aligned}
6 \mathrm{~d} \Delta \wedge * \bar{Z}= & * F+F \sin \zeta+12 \kappa Y-*\left(F \triangle_{2} Y\right) \\
& -2 \iota_{f} \bar{Z} .
\end{aligned}
$$

On the other hand, the fourth differential relation listed in (336) gives

$$
\mathrm{d}(* \bar{Z})=-2 F \sin \zeta-8 \kappa Y+*\left(F \triangle_{2} Y\right)+2 \iota_{f} \bar{Z} .
$$

Adding (369) and (370) gives (368). This finishes the proof of (353).

Proof of Relation (354). Relation (354) is obviously equivalent to

$$
3(\sin \zeta) \mathrm{d} \Delta+\mathrm{d} \sin \zeta=f-4 \kappa \bar{K}
$$

To prove (371), notice that the Hodge dual of the eighth algebraic constraint listed in (331) (i.e., the eight relation in (332)), after multiplication by 3 , gives

$$
3(\sin \zeta) \mathrm{d} \Delta=f-6 \kappa \bar{K}-\frac{1}{2} \iota_{* \bar{Z}} F
$$

On the other hand, the first differential constraint listed in (334) gives

$$
\mathrm{d} \sin \zeta=2 \kappa \bar{K}+\frac{1}{2} \iota_{*} \bar{Z} F
$$

Adding (372) and (373) gives (371), which finishes the proof of relation (354).

Proof of Relation (355). Using the first relation in (336) and first relation in (332) one finds

$$
12 \mathrm{~d} \Delta \wedge * \bar{K}+\mathrm{d} * \bar{K}=8 \kappa \sin \zeta \nu
$$

which, when expressing the volume form $v$ in terms of $\mathrm{vol}_{7}$ defined in (356), leads to (355).

\section{Conclusions and Further Directions}

We showed that geometric algebra techniques can be used to give a highly synthetic, conceptually transparent, and computationally efficient reformulation of the constrained Killing pinor equations, which constitute the condition that a flux background preserves a given amount of supersymmetry. This formulation clearly displays the algebraic and differential structure governing the supersymmetry conditions, leading to a description which opens the way for unified studies of flux backgrounds aimed at uncovering their deeper structure. We showed that our general formalism recovers results and methods which were used in [3] and therefore that it provides a powerful way to extend them. Our formulation is highly amenable to implementation in various symbolic computational packages specialized in tensor algebra, and we touched on two particular implementations which we have carried out using Ricci [33] in Mathematica ${ }^{\circledR}$, as well as Cadabra [34].

Here we illustrated our approach with the case of the most general compactifications of $M$-theory which preserve $\mathcal{N}=1$ supersymmetry in three dimensions [3, 4], showing how the results derived through different methods in loc. cit. can be recovered through our techniques. We stress that the methods introduced in this paper have much wider applicability than the example considered in Section 6, leading to promising new directions in the study of supergravity backgrounds and supergravity actions. In particular, we believe that many computationally difficult issues in the subject could be understood much better by using such techniques. The connection with a certain form of geometric quantization (which we have only touched upon) also leads to interesting ideas, problems, and directions for further research, which are currently under investigation. Further applications of our approach can be found in [35-38].

\section{Appendix}

\section{A. Identities Satisfied by the Covariant Derivative of Pinors}

Let $(M, g)$ be a pseudo-Riemannian manifold endowed with a local coordinate system $\left(x^{m}\right)$ and a local pseudoorthonormal frame $\left(e_{a}\right)$ (vielbein) of $(T M, g)$, both defined above an open set $U \subset M$. In this Appendix, both $m$ and $a$ run from 1 to $\operatorname{dim} M$. As usual, pseudo-orthonormality of $e_{a}$ means $g\left(e_{a}, e_{b}\right)=\eta_{a b}$, where $\eta_{a b}$ is a diagonal matrix all of whose diagonal entries equal +1 or -1 . We let $e^{a}$ denote the dual coframe of $M$, defined through $e^{a}\left(e_{b}\right)=\delta_{b}^{a}$; it is a local frame of $T^{*} M$ which is pseudo-orthonormal with respect to the metric $\hat{g}$ induced on $T^{*} M$; that is, $\hat{g}\left(e^{a}, e^{b}\right)=\eta^{a b}$ where $\eta^{a b} \eta_{b c}=\delta_{c}^{a}$. We have $\partial_{m} \stackrel{\text { def }}{=} \partial / \partial x^{m}=e_{m}^{a}(x) e_{a}$ and $e_{a}=e_{a}^{m}(x) \partial_{m}$ for some locally defined functions $e_{m}^{a}, e_{a}^{m}$ which satisfy $e_{m}^{a} e_{b}^{m}=\delta_{b}^{a}$ and $e_{m}^{a} e_{a}^{n}=\delta_{m}^{n}$. This implies $g\left(e_{a}, e_{b}\right)=$ $e_{a}^{m} e_{b}^{n} g_{m n}=\eta_{a b}$ and $g\left(\partial_{m}, \partial_{n}\right)=e_{m}^{a} e_{n}^{b} \eta_{a b}=g_{m n}$. Any tensor field $t \in \Gamma\left(T M^{\otimes p} \otimes\left(T^{*} M\right)^{\otimes q}\right)$ of type $(p, q)$ expands as follows:

$$
\begin{aligned}
t & ={ }_{U} t_{n_{1} \cdots n_{q}}^{m_{1} \cdots m_{p}} \partial_{m_{1}} \otimes \cdots \otimes \partial_{m_{p}} \otimes \mathrm{d} x^{n_{1}} \otimes \cdots \otimes \mathrm{d} x^{n_{q}} \\
& =t_{b_{1} \cdots b_{q}}^{a_{1} \cdots a_{p}} e_{a_{1}} \otimes \cdots \otimes e_{a_{p}} \otimes e^{b_{1}} \otimes \cdots \otimes e^{b_{q}},
\end{aligned}
$$


where the locally defined coefficient functions $t_{n_{1} \cdots n_{q}}^{m_{1} \cdots m_{p}}$ and $t_{b_{1} \cdots b_{q}}^{a_{1} \cdots a_{p}}$ are related through

$$
\begin{aligned}
& t_{b_{1} \cdots b_{q}}^{a_{1} \cdots a_{p}}=e_{m_{1}}^{a_{1}} \cdots e_{m_{p}}^{a_{p}} e_{b_{1}}^{n_{1}} \cdots e_{b_{q}}^{n_{q}} t_{n_{1} \cdots n_{q}}^{m_{1} \cdots m_{p}} \Longleftrightarrow \\
& t_{n_{1} \cdots n_{q}}^{m_{1} \cdots m_{p}}=e_{a_{1}}^{m_{1}} \cdots e_{a_{p}}^{m_{p}} e_{n_{1}}^{b_{1}} \cdots e_{n_{q}}^{b_{q}} t_{b_{1} \cdots b_{q}}^{a_{1} \cdots a_{p}} .
\end{aligned}
$$

Here and below, indices denoted by letters chosen from the middle of the Latin alphabet refer to the coordinate frame defined by $\left(\partial_{m}\right)$ while indices denoted by letters chosen from the beginning of the Latin alphabet refer to the local pseudoorthonormal frame (vielbein) defined by $\left(e_{a}\right)$.

A differential $k$-form $\omega \in \Omega^{k}(M)$ expands locally as in (2). Similarly, a polyvector field $\alpha \in \Gamma\left(M, \wedge^{k} T M\right)$ expands locally as follows:

$$
\alpha={ }_{U} \frac{1}{k !} \alpha^{a_{1} \cdots a_{k}}(x) e_{a_{1}} \wedge \cdots \wedge e_{a_{k}}
$$

with coefficients functions which are totally antisymmetric in the indices.

Let $\nabla$ be the Levi-Civita connection of $(M, g)$. Its Christoffel symbols $\Gamma_{m n}^{\rho}$ in the given local coordinates are defined through $\nabla_{m}\left(\partial_{n}\right)=\Gamma_{m n}^{\rho} \partial_{\rho}$, while its coefficients $\Omega_{m a}^{b}$ with respect to the given coordinate system and vielbein are determined by the expansion $\nabla_{m}\left(e_{a}\right)=\Omega_{m a}^{b} e_{b}$. Here and below, we set $\nabla_{m} \stackrel{\text { def }}{=} \nabla_{\partial_{m}}$. The two sets of connection coefficients are related through

$$
\begin{aligned}
\Omega_{m b}^{a} & =e_{n}^{a} e_{b}^{\lambda} \Gamma_{m \lambda}^{n}-e_{b}^{\lambda} \partial_{m} e_{\lambda}^{a} \Longleftrightarrow \\
\Gamma_{m \lambda}^{n} & =e_{a}^{n} e_{\lambda}^{b} \Omega_{m b}^{a}+e_{a}^{n} \partial_{m} e_{\lambda}^{a} .
\end{aligned}
$$

The fact that $\nabla$ is torsion-free amounts to the conditions

$$
\Gamma_{m n}^{\rho}=\Gamma_{n m}^{\rho} \Longleftrightarrow \Omega_{m a b}=-\Omega_{m b a},
$$

where $\Omega_{m a b}$ is defined through

$$
\Omega_{m a b} \stackrel{\text { def }}{=} \eta_{a c} \Omega_{m b}^{c}=g\left(e_{a}, \nabla_{m} e_{b}\right)=-g\left(\nabla_{m} e_{a}, e_{b}\right) .
$$

With respect to the vielbein, the covariant derivative of a $(p, q)$-tensor (A.1) takes the form

$$
\nabla_{m} t=\left(\nabla_{m} t\right)_{b_{1} \cdots b_{q}}^{a_{1} \cdots a_{p}} e_{a_{1}} \otimes \cdots \otimes e_{a_{p}} \otimes e^{b_{1}} \otimes \cdots \otimes e^{b_{q}},
$$

where

$$
\begin{gathered}
\left(\nabla_{m} t\right)_{b_{1} \cdots b_{q}}^{a_{1} \cdots a_{p}} \stackrel{\text { def }}{=} t_{b_{1} \cdots b_{q} ; m}^{a_{1} \cdots a_{p}} \\
=\partial_{m} t_{b_{1} \cdots b_{q}}^{a_{1} \cdots a_{p}}+\sum_{s=1}^{p} \Omega_{m a}^{a_{s}} t_{b_{1} \cdots b_{q}}^{a_{1} \cdots a_{s-1}, a, a_{s+1} \cdots a_{p}} \\
-\sum_{t=1}^{q} \Omega_{m b_{t}}^{a} t_{b_{1} \cdots b_{t-1}, a, b_{t+1} \cdots b_{q}}^{a_{1} \cdots a_{p}}
\end{gathered}
$$

For a differential form (2), this gives $\nabla_{m} \omega=\left(\nabla_{m} \omega\right)_{b_{1} \cdots b_{k}} e^{b_{1} \cdots b_{k}}$, with

$$
\begin{aligned}
\left(\nabla_{m} \omega\right)_{b_{1} \cdots b_{k}} & =\omega_{b_{1} \cdots b_{k} ; m} \\
& =\partial_{m} \omega_{b_{1} \cdots b_{k}}-\sum_{s=1}^{k} \Omega_{m b_{s}}^{a} \omega_{b_{1} \cdots b_{t-1}, a, b_{t+1} \cdots b_{k}},
\end{aligned}
$$

while for a polyvector field (A.3), we find $\nabla_{m} \alpha=$ $\left(\nabla_{m} \alpha\right)^{a_{1} \cdots a_{k}} e_{a_{1}} \wedge \cdots \wedge e_{a_{k}}$, with

$$
\begin{aligned}
& \left(\nabla_{m} \alpha\right)^{a_{1} \cdots a_{k}} \stackrel{\text { def }}{=} \alpha_{; m}^{a_{1} \cdots a_{p}} \\
& \quad=\partial_{m} \alpha^{a_{1} \cdots a_{k}}+\sum_{s=1}^{k} \Omega_{m a}^{a_{s}} \alpha^{a_{1} \cdots a_{s-1}, a, a_{s+1} \cdots a_{k}}
\end{aligned}
$$

Let $S$ be a pin bundle over $M$. Recall that the connection $\nabla^{S}$ induced by the Levi-Civita connection $\nabla$ takes the form

$$
\begin{aligned}
& \nabla_{m}^{S}=\partial_{m}+\widehat{\Omega}_{m} \\
& \text { where } \widehat{\Omega}_{m}=\frac{1}{4} \Omega_{m a b} \gamma^{a b}=\frac{1}{4} g\left(e_{a}, \nabla_{m} e_{b}\right) \gamma^{a b} \in \Gamma(M, \text { End }(S))
\end{aligned}
$$

this acts on sections of $S$ in the obvious manner. Here, $\gamma^{a} \in$ $\Gamma(M, \operatorname{End}(S))$ are the gamma operators associated with the coframe $\left(e^{a}\right)$, which satisfy

$$
\left[\gamma^{a}, \gamma^{b}\right]_{+, \circ}=2 \eta^{a b}
$$

We will also use the operators $\gamma_{a} \stackrel{\text { def }}{=} \eta_{a b} \gamma^{b}$, which satisfy $\left[\gamma_{a}, \gamma_{b}\right]_{+, 0}=2 \eta_{a b}$. In what follows, we recall some basic properties of $\nabla^{S}$.

Algebraic Identities. Let $V$ be a finite-dimensional $\mathbb{K}$-vector space. For $X, Y \in \operatorname{End}(V)$, we set $[X, Y]_{\epsilon} \stackrel{\text { def }}{=} X Y+\epsilon Y X$, where $\epsilon \in\{-1,+1\}$, so that $[X, Y]_{+1} \stackrel{\text { def }}{=}[X, Y]_{+, \circ}$ is the anticommutator of $X$ with $Y$, while $[X, Y]_{-1} \stackrel{\text { def }}{=}[X, Y]_{-, o}$ is the commutator. We start with the following trivial observation.

Lemma A.1. For any $A, B, C \in \operatorname{Mat}(n, \mathbb{K})$ and any $\epsilon \in$ $\{-1,+1\}$, we have

$$
[A B, C]_{\epsilon}=A[B, C]_{\epsilon}-\epsilon[A, C]_{\epsilon} B
$$

Proposition A.2. The following identities hold for all $p \neq q$ :

$$
\begin{aligned}
{\left[\gamma^{p} \circ \gamma^{q}, \gamma^{a_{1} \cdots a_{k}}\right]_{-, \circ}=} & 2 \sum_{s=1}^{k} \eta^{q a_{s}} \gamma^{a_{1} \cdots a_{s-1}} p a_{s+1} \cdots a_{k} \\
& -(p \longleftrightarrow q), \\
{\left[\gamma^{p} \circ \gamma^{q}, \gamma_{a_{1} \cdots a_{k}}\right]_{-, \circ}=} & 2 \sum_{s=1}^{k} \delta_{a_{s}}^{q} \eta^{p p^{\prime}} \gamma_{a_{1} \cdots a_{s-1}} p^{\prime} a_{s+1} \cdots a_{k} \\
& -(p \longleftrightarrow q) .
\end{aligned}
$$


Proof. We prove only the first identity, since it immediately implies the second upon lowering indices with $\eta$. Applying the lemma with $\epsilon=(-1)^{k-1}$, we find

$$
\begin{aligned}
{\left[\gamma^{p} \circ \gamma^{q}, \gamma^{a_{1} \cdots a_{k}}\right]_{-, \circ}=} & \gamma^{p} \circ\left[\gamma^{q}, \gamma^{a_{1} \cdots a_{k}}\right]_{(-1)^{k-1}, \circ} \\
& +(-1)^{k}\left[\gamma^{p}, \gamma^{a_{1} \cdots a_{k}}\right]_{(-1)^{k-1},} \\
& \circ \gamma^{q} .
\end{aligned}
$$

A simple computation (or a mathematical induction argument) gives

$$
\left[\gamma^{p}, \gamma^{a_{1} \cdots a_{k}}\right]_{(-1)^{k-1}, \circ}=2 \sum_{s=1}^{k}(-1)^{s-1} \eta^{p a_{s}} \gamma^{a_{1} \cdots \widehat{a}_{s} \cdots a_{k}},
$$

where the hat indicates that the corresponding index is missing. Using this equation and its counterpart with $p$ replaced by $q$ in (A.16) gives

$$
\begin{aligned}
& {\left[\gamma^{p} \circ \gamma^{q}, \gamma^{a_{1} \cdots a_{k}}\right]_{-, \circ}} \\
& =2 \sum_{s=1}^{k}(-1)^{s-1} \eta^{q a_{s}} \gamma^{p} \circ \gamma^{a_{1} \cdots \widehat{a}_{s} \cdots a_{k}} \\
& \quad+2 \sum_{s=1}^{k}(-1)^{s-1} \eta^{p a_{s}} \gamma^{a_{1} \cdots \widehat{a}_{s} \cdots a_{k}} \circ \gamma^{q} .
\end{aligned}
$$

We next use the following identities, which can be checked by mathematical induction ${ }^{9}$ :

$$
\begin{aligned}
& \gamma^{p} \circ \gamma^{a_{1} \cdots a_{s} \cdots a_{k}} \\
&=(-1)^{s-1} \gamma^{a_{1} \cdots a_{s-1}} p a_{s+1} \cdots a_{k} \\
& \quad+2 \sum_{t=1}^{s-1}(-1)^{t-1} \eta^{p a_{t}} \gamma^{p} \circ \gamma^{a_{1} \cdots \widehat{a}_{t} \cdots \widehat{a}_{s} \cdots a_{k}}, \\
& \gamma^{a_{1} \cdots \widehat{a}_{s} \cdots a_{k}} \circ \gamma^{q} \\
&=(-1)^{k-s} \gamma^{a_{1} \cdots a_{s-1} q a_{s+1} \cdots a_{k}} \\
& \quad+2 \sum_{t=s+1}^{k}(-1)^{k-t} \eta^{q a_{t}} \gamma^{q} \circ \gamma^{a_{1} \cdots \widehat{a}_{s} \cdots \widehat{a}_{t} \cdots a_{k}} .
\end{aligned}
$$

Inserting these in (A.18) gives

$$
\begin{aligned}
& {\left[\gamma^{p} \circ \gamma^{q}, \gamma^{a_{1} \cdots a_{k}}\right]_{-, \circ}=} 2 \sum_{s=1}^{k} \eta^{q a_{s}} \gamma^{a_{1} \cdots a_{s-1}} p a_{s+1} \cdots a_{k} \\
&-(p \longleftrightarrow q)+T
\end{aligned}
$$

where the term $T$ is given by

$$
T=4\left(T_{1}-T_{2}\right),
$$

with $T_{1}=\sum_{1 \leq t<s \leq k}(-1)^{s+t} \eta^{p a_{t}} \eta^{q a_{s}} \gamma^{a_{1} \cdots \hat{a}_{t} \cdots \widehat{a}_{s} \cdots a_{k}}$ and $T_{2}=$ $\sum_{1 \leq s<t \leq k}(-1)^{s+t} \eta^{q a_{t}} \eta^{p a_{s}} \gamma^{a_{1} \cdots \widehat{a}_{s} \cdots \widehat{a}_{t} \cdots a_{k}}$. Since $T_{2}=\left.T_{1}\right|_{p \leftrightarrow q}$, we have $T=0$ and the first relation (A.14) is proved.
Proposition A.2 has the following immediate consequence, which follows by using the antisymmetry property (A.5) of $\Omega_{m p q}$.

Proposition A.3. The following identities hold:

$$
\begin{aligned}
{\left[\nabla_{m}^{S}, \gamma^{a_{1} \cdots a_{k}}\right]_{-, \circ} } & =\frac{1}{4} \Omega_{m b c}\left[\gamma^{b c}, \gamma^{a_{1} \cdots a_{k}}\right]_{-, \circ} \\
& =-\sum_{s=1}^{k} \Omega_{m}{ }^{a_{s}} \gamma^{a_{1} \cdots a_{s-1}} p a_{s+1} \cdots a_{k}, \\
{\left[\nabla_{m}^{S}, \gamma_{a_{1} \cdots a_{k}}\right]_{-, \circ} } & =\frac{1}{4} \Omega_{m b c}\left[\gamma^{b c}, \gamma_{a_{1} \cdots a_{k}}\right]_{-, \circ} \\
& =+\sum_{s=1}^{k} \Omega_{m}{ }^{p}{ }_{a_{s}} \gamma_{a_{1} \cdots a_{s-1}} p a_{s+1} \cdots a_{k} .
\end{aligned}
$$

Consider an arbitrary $k$-form $\omega$ as in (2) and an arbitrary polyvector field $\alpha$ as in (A.3). We define endomorphisms of the pin bundle $S$ via

$$
\begin{aligned}
\gamma(\omega) & =\frac{1}{k !} \omega_{a_{1} \cdots a_{k}} \gamma^{a_{1} \cdots a_{k}}, \\
\gamma\left(\nabla_{m} \omega\right) & =\frac{1}{k !}\left(\nabla_{m} \omega\right)_{a_{1} \cdots a_{k}} \gamma^{a_{1} \cdots a_{k}}, \\
\gamma(\alpha) & =\frac{1}{k !} \alpha^{a_{1} \cdots a_{k}} \gamma_{a_{1} \cdots a_{k}}, \\
\gamma\left(\nabla_{m} \alpha\right) & =\frac{1}{k !}\left(\nabla_{m} \alpha\right)^{a_{1} \cdots a_{k}} \gamma_{a_{1} \cdots a_{k}} .
\end{aligned}
$$

Proposition A.3 implies the following.

Proposition A.4. $\nabla^{S}$ satisfies the following identities for any differential form $\omega$ and any polyvector field $\alpha$ :

$$
\begin{aligned}
& {\left[\nabla_{m}^{S}, \gamma(\omega)\right]_{-, \circ}=\gamma\left(\nabla_{m} \omega\right),} \\
& {\left[\nabla_{m}^{S}, \gamma(\alpha)\right]_{-, \circ}=\gamma\left(\nabla_{m} \alpha\right) .}
\end{aligned}
$$

Proof. Relations (A.24) follow by using Proposition A.4, (A.9), and (A.10) as well as an obvious relabeling of dummy indices.

Observation. The first identity in Proposition A.4 is the wellknown statement that $\nabla^{S}$ is a Clifford connection on $S$ in the sense typically used in spin geometry (see, e.g., [39]).

\section{B. Component Approach to Pinor Bilinears}

In this appendix, we show that the abstract equations (224) and (246) are equivalent to a set of equations which were found in [3] via component calculations pertaining to the particular case considered in loc. cit.

B.1. Alternate Form of the Algebraic Constraints. Taking linear combinations of (223) (equivalently, using the fact that $\left.\mathscr{K}\left(L_{\check{Q}}\right) \cap \mathscr{K}\left(R_{\tau_{\mathscr{B}}(\check{Q})}\right)=\mathscr{K}\left(L_{\check{Q}}+R_{\tau_{\mathscr{B}}(\check{Q})}\right) \cap \mathscr{K}\left(L_{\check{Q}}-R_{\tau_{\mathscr{B}}(\check{Q})}\right)\right)$ 
shows that the algebraic constraints derived in Section 5 can also be written in the following form:

$$
\begin{aligned}
\mathscr{B} & \left(\xi,\left(Q^{t} \circ \gamma_{a_{1} \cdots a_{k}} \pm \gamma_{a_{1} \cdots a_{k}} \circ Q\right) \xi^{\prime}\right) \\
& =\xi^{t}\left(Q^{t} \circ \gamma_{a_{1} \cdots a_{k}} \pm \gamma_{a_{1} \cdots a_{k}} \circ Q\right) \xi^{\prime}=0 .
\end{aligned}
$$

These equations are equivalent-for the particular case considered there-with a set of conditions used in [3]. Starting from equation (2.26) of that reference, let us show that the "useful relations" of [3, Appendix C] are equivalent to our algebraic constraints. Recall that [3] deals with the case of Majorana spinors $\xi$ in eight Euclidean dimensions, a case which was also the subject of our application in Section 6. As explained in that section, we prefer to work directly with $\xi$ rather than with the quantities $\varepsilon_{+}=(1 / \sqrt{2}) \xi$ and $\varepsilon_{-}=$ $(1 / \sqrt{2}) \gamma^{(9)} \xi$ used in loc. cit. (which provide a redundant parameterization of $\xi$ ).

When expressed in terms of $\xi$, (2.26) of [3] is equivalent to $Q \xi=0$, where $Q$ is given in (304). Choosing a local frame of $S$, we can think of $Q$ as a locally defined matrix-valued function and of $\xi$ as a column matrix with entries given by locally defined smooth functions. When $Q \xi=0$, we also have $\xi^{t} Q^{t}=0$, where

$$
\begin{aligned}
Q^{t}= & \frac{1}{2}\left(\partial_{n} \Delta\right) \gamma^{n}+\frac{1}{6} f_{n} \gamma^{n} \circ \gamma^{(9)}-\frac{1}{288} F_{n p q r} \gamma^{n p q r} \\
& -\kappa \gamma^{(9)}
\end{aligned}
$$

The two equations $Q \xi=0$ and $\xi^{t} Q^{t}=0$ imply the relations

$$
\begin{gathered}
\xi^{t} T \circ Q \xi=0, \\
\xi^{t} Q^{t} \circ T \xi=0,
\end{gathered}
$$

where $T \in \operatorname{Mat}(16, \mathbb{R}) \approx \mathrm{Cl}(8,0)$ is a general Clifford matrix. Using relations (308) and the fact that $\gamma^{(9)}$ anticommutes with $\gamma^{1}, \ldots, \gamma^{8}$, it is easy to check that the "useful relations" (C.1)(C.3) given in Appendix C of [3] take the following form when expressed in terms of $\xi$ :

$$
\begin{aligned}
& \frac{1}{288} F_{p q r s} \xi^{t}\left[\gamma^{p q r s}, T\right]_{\mp} \xi-\frac{1}{2}\left(\partial_{m} \Delta\right) \xi^{t}\left[\gamma^{m}, T\right]_{\mp} \xi \\
& +\kappa \xi^{t}\left[\gamma^{(9)}, T\right]_{\mp} \xi-\frac{1}{6} f_{m} \xi^{t}\left[\gamma_{m} \gamma^{(9)}, T\right]_{ \pm} \xi=0, \\
& \frac{1}{288} F_{p q r s} \xi^{t}\left[\gamma^{p q r s}, T \gamma^{(9)}\right]_{\mp} \xi \\
& -\frac{1}{2}\left(\partial_{m} \Delta\right) \xi^{t}\left[\gamma^{m}, T \gamma^{(9)}\right]_{\mp} \xi+\kappa \xi^{t}\left[\gamma^{(9)}, T \gamma^{(9)}\right]_{\mp} \xi \\
& -\frac{1}{6} f_{m} \xi^{t}\left[\gamma_{m} \gamma^{(9)}, T \gamma^{(9)}\right]_{ \pm} \xi=0 .
\end{aligned}
$$

It is now clear that the first identity in (B.5) is equivalent to the difference (B.3) - (B.4) and the second is equivalent to the sum (B.3) + (B.4), while the two identities in (B.6) are equivalent to (B.3) $\mp$ (B.4) where $T$ is replaced by $T \gamma^{(9)}$. One can easily check that the third and fourth identities are not independent, being equivalent to the first two. We conclude that the useful relations of [3] are equivalent to the particular incarnation of our algebraic constraints for the case considered in loc. cit.

B.2. Alternate Derivation of the Differential Constraints. For simplicity, let us consider only the case $\mathbb{K}=\mathbb{R}$. Recall that the pin bundle $S$ of a pseudo-Riemannian manifold $(M, g)$ is endowed with admissible bilinear pairings $\mathscr{B}$, which, in particular, satisfy (174).

Since $\widehat{\Omega}_{m}^{t}=-\widehat{\Omega}_{m}$, we find that $\widehat{\Omega}_{m}$ is anti-self-adjoint with respect to the pairing $\mathscr{B}$, which means that the pin covariant derivative $\nabla^{S}$ induced by the Levi-Civita connection of $(M, g)$ is compatible with $\mathscr{B}$ in the sense that this pairing is $\nabla^{S}$-flat:

$$
\begin{aligned}
& \partial_{m} \mathscr{B}\left(\xi, \xi^{\prime}\right)=\mathscr{B}\left(\nabla_{m}^{S} \xi, \xi^{\prime}\right)+\mathscr{B}\left(\xi, \nabla_{m}^{S} \xi^{\prime}\right) \\
& \forall \xi, \xi^{\prime} \in \Gamma(M, S) .
\end{aligned}
$$

The deformed pin connection takes the form $D_{m}=\nabla_{m}^{S}+A_{m}=$ $\partial_{m}+\widehat{\Omega}_{m}+A_{m}$. Since $A_{m}^{t}=-A_{m}$, it follows that $A_{m}$ (and thus also $\widehat{\Omega}_{m}+A_{m}$ ) is again anti-self-adjoint with respect to the scalar product on $S$; as a consequence, the deformed pin connection is also compatible with this pairing:

$$
\begin{aligned}
& \partial_{m} \mathscr{B}\left(\xi, \xi^{\prime}\right)=\mathscr{B}\left(D_{m} \xi, \xi^{\prime}\right)+\mathscr{B}\left(\xi, D_{m} \xi^{\prime}\right) \\
& \forall \xi, \xi^{\prime} \in \Gamma(M, S) .
\end{aligned}
$$

Replacing $\xi^{\prime}$ with $T \xi^{\prime}$ in the last equation (where $T \in$ $\Gamma\left(M, \operatorname{End}_{\mathbb{R}}(S)\right)$ is arbitrary) gives

$$
\begin{aligned}
\partial_{m} \mathscr{B}\left(\xi, T \xi^{\prime}\right)= & \mathscr{B}\left(D_{m} \xi, T \xi^{\prime}\right) \\
& +\mathscr{B}\left(\xi,\left[D_{m}, T\right]_{-, \circ} \xi^{\prime}\right) \\
& +\mathscr{B}\left(\xi, T D_{m} \xi^{\prime}\right), \\
& \forall \xi, \xi^{\prime} \in \Gamma(M, S),
\end{aligned}
$$

which immediately implies the following statement.

Lemma B.1. When $D_{m} \xi=D_{m} \xi^{\prime}=0$, we have

$$
\begin{aligned}
\partial_{m} \mathscr{B}\left(\xi, T \xi^{\prime}\right)=\mathscr{B}\left(\xi,\left[D_{m}, T\right]_{-, o} \xi^{\prime}\right), & \\
& \forall T \in \Gamma\left(M, \operatorname{End}_{\mathbb{R}}(S)\right) .
\end{aligned}
$$

Let now $\check{\mathbf{E}}^{(k)} \in \Omega^{k}(M)$ be a $k$-form defined through

$$
\begin{aligned}
\check{\mathbf{E}}_{a_{1} \cdots a_{k}}^{(k)} \stackrel{\text { def }}{=} \mathscr{B}\left(\xi, \gamma_{a_{1} \cdots a_{k}} \xi^{\prime}\right) \Longrightarrow \\
\check{\mathbf{E}}^{(k)}=\mathscr{B}\left(\xi, \gamma_{a_{1} \cdots a_{k}} \xi^{\prime}\right) e^{a_{1} \cdots a_{k}},
\end{aligned}
$$

where $\left(e_{a}\right)$ is a local pseudo-orthonormal frame of $(M, g)$ and $\gamma_{a}=\gamma\left(\left(e_{a}\right)_{\sharp}\right)=\eta_{a b} \gamma^{b}$. 
Proposition B.2. When $D_{m} \xi=D_{m} \xi^{\prime}=0$, we have

$$
\begin{aligned}
\left(\nabla_{m} \check{\mathbf{E}}^{(k)}\right)_{a_{1} \cdots a_{k}} & =\check{\mathbf{E}}_{a_{1} \cdots a_{k} ; m}^{(k)} \\
& =\mathscr{B}\left(\xi,\left[A_{m}, \gamma_{a_{1} \cdots a_{k}}\right]_{-, \circ} \xi^{\prime}\right) .
\end{aligned}
$$

Proof. Applying Lemma B.1 to $T=\gamma_{a_{1} \cdots a_{k}}$ gives

$$
\begin{aligned}
& \partial_{m} \check{\mathbf{E}}_{a_{1} \cdots a_{k}}^{(k)}=\mathscr{B}\left(\xi,\left[D_{m}, \gamma_{a_{1} \cdots a_{k}}\right]_{-, o} \xi^{\prime}\right) \\
& =\mathscr{B}\left(\xi,\left[\nabla_{m}^{S}, \gamma_{a_{1} \cdots a_{k}}\right]_{-, o} \xi^{\prime}\right) \\
& +\mathscr{B}\left(\xi,\left[A_{m}, \gamma_{a_{1} \cdots a_{k}}\right]_{-, o} \xi^{\prime}\right)
\end{aligned}
$$

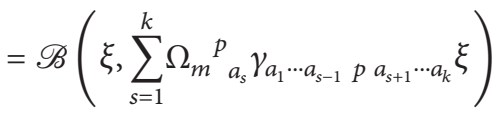

$$
\begin{aligned}
& +\mathscr{B}\left(\xi,\left[A_{m}, \gamma_{a_{1} \cdots a_{k}}\right]_{-, o} \xi^{\prime}\right),
\end{aligned}
$$

where we used the second identity stated in Proposition A.3 of Appendix A. The conclusion follows upon moving the first term of the last expression to the left hand side and applying (A.9).

\section{Competing Interests}

The authors declare that they have no competing interests.

\section{Acknowledgments}

This work was supported by the CNCS projects PN-II-RUTE (Contract no. 77/2010), PN-II-ID-PCE (Contract nos. 50/2011 and 121/2011), and PN 093701 02/2009. The work of Calin-Iuliu Lazaroiu was also supported by the Research Center Program of IBS (Institute for Basic Science) in Korea (Grant CA1205-01). Calin-Iuliu Lazaroiu and Elena-Mirela Babalic thank the Center for Geometry and Physics, Institute for Basic Science and Pohang University of Science and Technology (POSTECH), Korea, and especially Jae-Suk Park for providing excellent conditions at various stages during the preparation of this work, through the research visitor program affiliated with Grant no. CA1205-1. The Center for Geometry and Physics is supported by the Government of Korea through the Research Center Program of IBS (Institute for Basic Science). Calin-Iuliu Lazaroiu also thanks Perimeter Institute for hospitality and for providing an excellent and stimulating research environment during the last stages of the preparation of this paper. Research at Perimeter Institute is supported by the Government of Canada through Industry Canada and by the Province of Ontario through the Ministry of Economic Development and Innovation. CalinIuliu Lazaroiu thanks Lilia Anguelova for interest and for stimulating discussions as well as for critical input during the final stages of this project. Ioana-Alexandra Coman acknowledges the student scholarship from the Dinu Patriciu Foundation "Open Horizons," which supported part of her studies.

\section{Endnotes}

1. They can, of course, also be formulated using complex Weyl spinors.

2. Indeed, the values of $\xi_{1}, \ldots, \xi_{s}$ at two points $x, y$ of $M$ are related through the parallel transport of $D$ along some curve connecting $x$ and $y$ in $M$. Since the parallel transport gives a linear isomorphism between the fibers $S_{x}$ and $S_{y}$ of $S$, it follows that any linear dependence relation within $\xi_{1}(x), \ldots, \xi_{s}(x)$ also holds-with the same coefficients-within $\xi_{1}(y), \ldots, \xi_{s}(y)$. Since $x$ and $y$ are arbitrary, this would give a linear dependence relation over $\mathbb{K}$ (i.e., with constant coefficients) between the globally defined sections $\xi_{1}, \ldots, \xi_{s}$, which contradicts our assumptions.

3. In general, we have $\iota_{\omega} \circ \iota_{\omega}=\wedge_{\omega} \circ \wedge_{\omega}=0$ for any $\omega \epsilon$ $\Omega_{\mathbb{K}}^{\text {odd }}(M)$.

4. Of course, we can view $\diamond$ as a section of the vector bundle $\operatorname{Hom}\left(\wedge T_{\mathbb{K}}^{*} M \otimes \wedge T_{\mathbb{K}}^{*} M, \wedge T_{\mathbb{K}}^{*} M\right)$

5. This is most easily explained in the case when $d=\operatorname{dim} M$ is even; namely, $d=2 r$. Then "vertical" fermionic Weyl quantization (in which one quantizes only along the odd directions of ПTM, while treating the body $M$ as classical) can be performed by choosing an almost complex structure on $M$, which induces a decomposition $T_{\mathbb{C}} M=W \oplus W^{*}$ of the complexified tangent bundle of $M$, with $W$ a complex vector bundle. This allows us to define fermionic Fock representations at each point $x \in M$ given by annihilation and creation operators $\mathbf{a}_{k} \stackrel{\text { def }}{=}(1 / \sqrt{2})\left(\gamma_{k}+i \gamma_{r+k}\right), \mathbf{a}_{k}^{\dagger} \stackrel{\text { def }}{=}(1 / \sqrt{2})\left(\gamma_{k}-i \gamma_{r+k}\right)$ (where $i=1 \cdots r)$ defined at $x$, with coherent states given by $|z\rangle_{x} \stackrel{\text { def }}{=} e^{-\sum_{k=1}^{d / 2} z^{k} \mathbf{a}_{k}^{\dagger}}|0\rangle_{x}$, where $|0\rangle_{x}$ is the vacuum at $x$ and $z^{k}=(1 / 2)\left(\zeta^{k}+i \zeta^{r+k}\right)$ are odd complex coordinates along the fibers of $\Pi T_{\mathbb{C}} M$. Identifying $\Omega_{\mathbb{C}}(M)$ with the algebra of complex functions on ПTM, we have $\mathbf{a}_{k}=\partial / \partial z^{k}$ and $\mathbf{a}_{k}^{\dagger}=\bar{z}^{k}$, so the bundle of spin Fock spaces can be identified with the subbundle $\wedge W^{*}$ of $\wedge T_{\mathbb{C}}^{*} M$. The star product $\star$ takes the form

$$
\begin{aligned}
f_{\omega \diamond \eta} & =f_{\omega} \star f_{\eta} \\
& =f_{\omega} \exp \left(g^{k \bar{l}} \frac{\overleftarrow{\partial}}{\partial z^{k}} \frac{\vec{\partial}}{\partial \bar{z}^{l}}+g^{\bar{k} l} \frac{\grave{\partial}}{\partial \bar{z}^{k}} \frac{\vec{\partial}}{\partial z^{l}}\right) f_{\eta},
\end{aligned}
$$

which agrees with (34).

6. This map can be defined naturally on the bundle of endomorphisms of any vector bundle, making no reference whatsoever to any bilinear pairing on the bundle.

7. The same is true for any metric but torsion-full connection.

8. Note that $D_{m} \otimes \mathrm{id}_{S}+\mathrm{id}_{S} \otimes D_{m}$ is the connection induced by $D_{m}$ on $S \otimes S$.

9. Notice that there is no Einstein summation over $p$ or $q$. 


\section{References}

[1] J. P. Gauntlett, D. Martelli, and D. Waldram, "Superstrings with intrinsic torsion," Physical Review D, vol. 69, no. 8, Article ID 086002, 27 pages, 2004.

[2] I. Agricola, "The Srni lectures on non-integrable geometries with torsion," Archivum Mathematicum, vol. 42, pp. 5-84, 2006.

[3] D. Martelli and J. Sparks, " $G$ structures, fluxes, and calibrations in M theory," Physical Review D, vol. 68, no. 8, Article ID 085014, 2003.

[4] D. Tsimpis, "M-theory on eight-manifolds revisited: $\mathcal{N}=1$ supersymmetry and generalized Spin(7) structures," Journal of High Energy Physics, vol. 2006, no. 4, article 027, 26 pages, 2006.

[5] F. Witt, "Generalised G(2)-manifolds," Communications in Mathematical Physics, vol. 265, no. 2, pp. 275-303, 2006.

[6] M. Gabella, D. Martelli, A. Passias, and J. Sparks, " $N=2$ supersymmetric $\mathrm{AdS}_{4}$ solutions of M-theory," Communications in Mathematical Physics, vol. 325, no. 2, pp. 487-525, 2014.

[7] H. B. Lawson and M.-L. Michelsohn, Spin Geometry, Princeton University Press, 1989.

[8] C. Chevalley, The Algebraic Theory of Spinors and Clifford Algebras: Collected Works, vol. 2 of edited by P. Cartier and C. Chevalley, Springer, 1996.

[9] M. Riesz, Clifford Numbers and Spinors: with Riesz's Private Lectures to E. Folke Bolinder and a Historical Review by Pertti Lounesto, Kluwer Academic Publishers Group, 1993.

[10] W. Graf, "Differential forms as spinors," Annales de l'Institut Henri Poincaré A, vol. 29, no. 1, pp. 85-109, 1978.

[11] D. Hestenes and G. Sobczyk, Clifford Algebra to Geometric Calculus: A Unified Language for Mathematics and Physics, Fundamental Theories of Physics, Kluwer, 1984.

[12] C. Doran and A. Lasenby, Geometric Algebra for Physicists, Cambridge University Press, 2003.

[13] P. Charlton, The geometry of pure spinors, with applications [Ph.D. thesis], Department of Mathematics, The University of Newcastle, Callaghan, Australia, 1997, http://csusap.csu.edu.au/ $\sim$ pcharlto/charlton_thesis.pdf.

[14] W. A. Rodrigues and E. C. de Oliveira, The Many Faces of Maxwell, Dirac and Einstein Equations: A Clifford Bundle Approach, vol. 722 of Lecture Notes in Physics, Springer, Berlin, Germany, 2007.

[15] G. Papadopoulos and D. Tsimpis, "The holonomy of the supercovariant connection and killing spinors," Journal of High Energy Physics, vol. 2003, no. 7, article 018, 28 pages, 2003.

[16] G. Papadopoulos and D. Tsimpis, "The holonomy of IIB supercovariant connection," Classical and Quantum Gravity, vol. 20, no. 20, article L253, 2003.

[17] R. L. Bryant, S.-S. Chern, R. B. Gardner, H. L. Goldschmidt, and P. A. Griffiths, Exterior Differential Systems, MSRI Publications 18, Springer, New York, NY, USA, 1991.

[18] A. Trautman, "Connections and the Dirac operator on spinor bundles," Journal of Geometry and Physics, vol. 58, no. 2, pp. 238-252, 2008

[19] H. Sati, "Geometry of Spin and Spinc structures in the M-theory partition function," Reviews in Mathematical Physics, vol. 24, no. 3, Article ID 1250005, 112 pages, 2012.

[20] A. C. Hirshfeld and P. Henselder, "Deformation quantization for systems with fermions," Annals of Physics, vol. 302, no. 1, pp. 5977, 2002.

[21] A. C. Hirshfeld, P. Henselder, and T. Spernat, "Cliffordization, spin, and fermionic star products," Annals of Physics, vol. 314, no. 1, pp. 75-98, 2004.
[22] P. Henselder, "Deformed geometric algebra and supersymmetric quantum mechanics," Physics Letters. A, vol. 363, no. 5-6, pp. 378-380, 2007.

[23] P. Henselder, "Geometric algebra and star products on the phase space," Annals of Physics, vol. 322, no. 5, pp. 1062-1095, 2007.

[24] T. Houri, D. Kubiznak, C. Warnick, and Y. Yasui, "Symmetries of the Dirac operator with skew-symmetric torsion," Classical and Quantum Gravity, vol. 27, no. 18, Article ID 185019, 2010.

[25] D. Kubiznak, C. M. Warnick, and P. Krtous, "Hidden symmetry in the presence of fluxes," Nuclear Physics. B, vol. 844, no. 2, pp. 185-198, 2011.

[26] M. Cariglia, P. Krtouš, and D. Kubizňák, "Commuting symmetry operators of the Dirac equation, Killing-Yano and SchoutenNijenhuis brackets," Physical Review D: Particles, Fields, Gravitation and Cosmology, vol. 84, no. 2, Article ID 024004, 2011.

[27] I. M. Benn and R. S. Tucker, An Introduction to spinors and Geometry with Applications in Physics, Adam Hilger, London, UK, 1987.

[28] S. Okubo, "Representations of Clifford algebras and its applications," Math. Jap., vol. 41, pp. 59-79, 1995.

[29] T. Friedrich and A. Trautman, "Spin spaces, Lipschitz groups, and spinor bundles," Annals of Global Analysis and Geometry, vol. 18, no. 3-4, pp. 221-240, 2000.

[30] D. V. Alekseevsky and V. Cortés, "Classification of $N$-(super)extended Poincaré algebras and bilinear invariants of the spinor representation of spin $(p, q)$," Communications in Mathematical Physics, vol. 183, no. 3, pp. 477-510, 1997.

[31] D. V. Alekseevsky, V. Cortés, C. Devchand, and A. van Proeyen, "Polyvector super-poincaré algebras," Communications in Mathematical Physics, vol. 253, no. 2, pp. 385-422, 2005.

[32] L.-S. Randriamihamison, "Identites de Fierz et formes bilinéaires dans les espaces spinoriels," Journal of Geometry and Physics, vol. 10, no. 1, pp. 19-35, 1992.

[33] J. M. Lee, D. Lear, J. Roth, J. Coskey, and L. Nave, "Ricci: A Mathematica package for doing tensor calculations in differential geometry," http://www.math.washington.edu/ lee/Ricci/ Manual.pdf.

[34] K. Peeters, "Introducing Cadabra: a symbolic computer algebra system for field theory problems," SPIN-06/46, ITP-UU-06/56, http://arxiv.org/abs/hep-th/0701238.

[35] E. M. Babalic and C. I. Lazaroiu, "Foliated eight-manifolds for M-theory compactification," Journal of High Energy Physics, vol. 1, article 140, 2015.

[36] E. M. Babalic and C. I. Lazaroiu, "Singular foliations for Mtheory compactification," Journal of High Energy Physics, vol. 2015, no. 3, article 116, 2015.

[37] C. I. Lazaroiu and E. M. Babalic, "Geometric algebra techniques in FLux compactifications (II)," Journal of High Energy Physics, vol. 2013, no. 6, article 054, 2013.

[38] C. I. Lazaroiu, E. M. Babalic, and I. A. Coman, "The geometric algebra of Fierz identities in arbitrary dimensions and signatures," Journal of High Energy Physics, vol. 2013, 156 pages, 2013.

[39] N. Berline, E. Getzler, and M. Vergne, Heat Kernels and Dirac Operators, vol. 298 of Grundlehren der Mathematischen Wissenschaften, Springer, New York, NY, USA, 1992. 

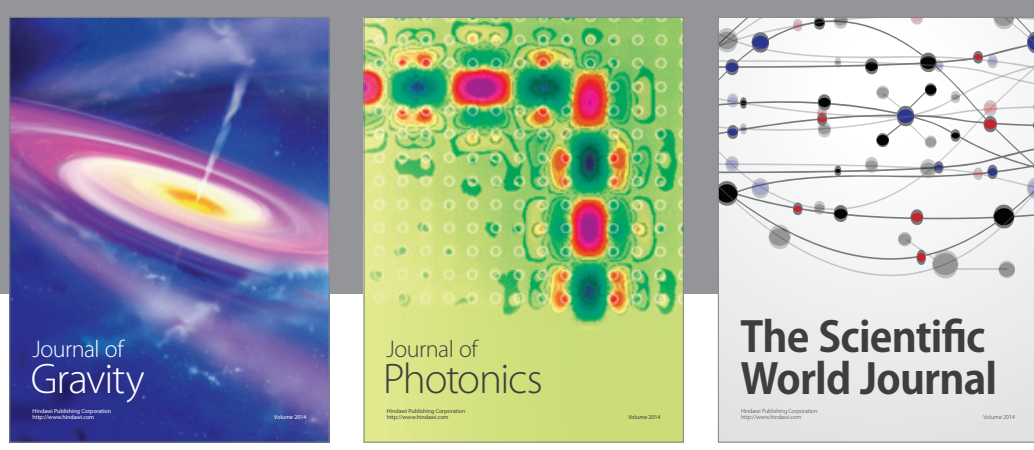

The Scientific World Journal
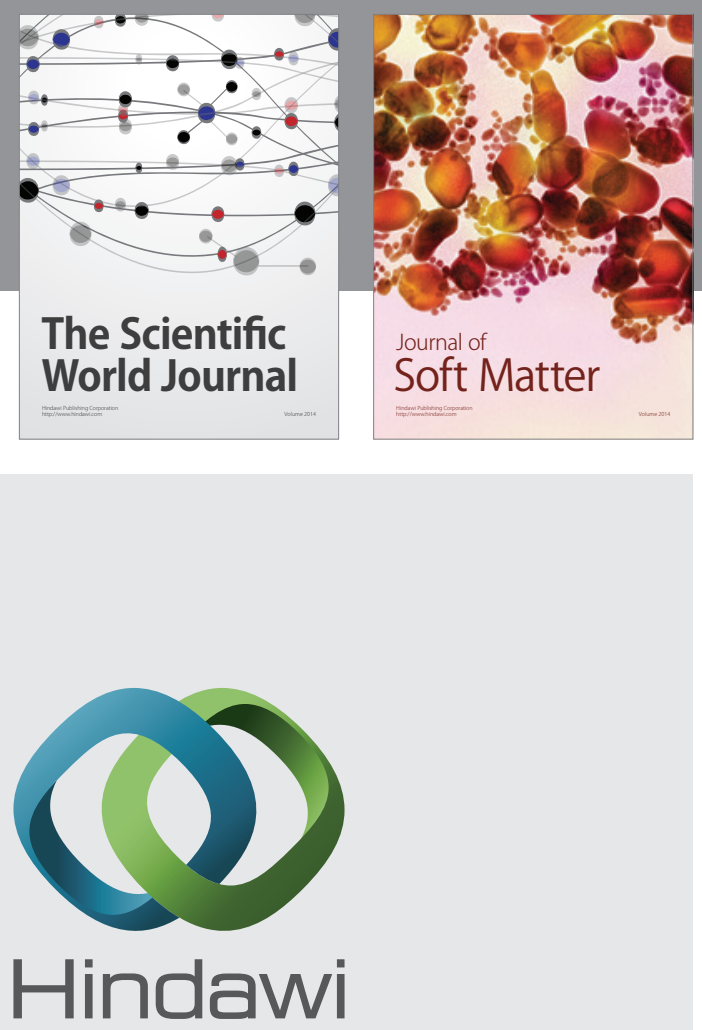

Submit your manuscripts at

http://www.hindawi.com

nternational Journal of

Statistical Mechanics
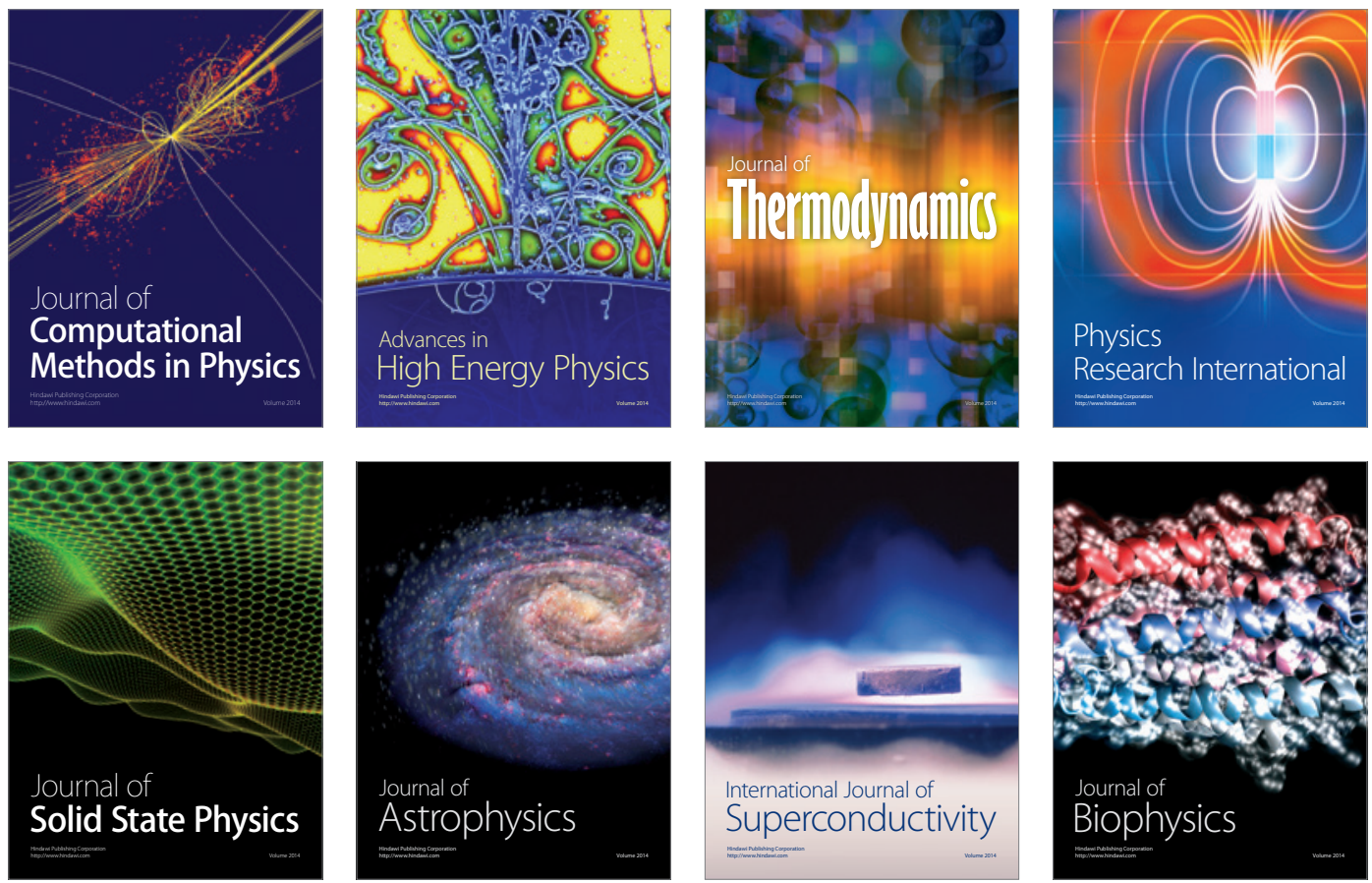
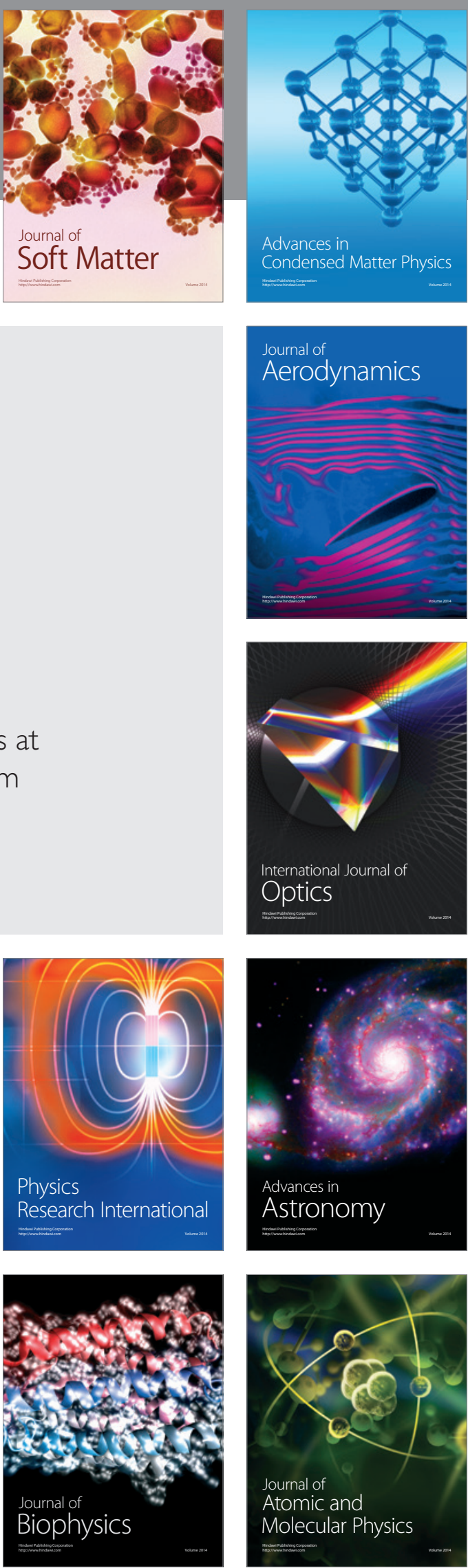